\title{
Security Design with Correlated Hidden Cash Flows: The Optimality of Performance Pricing*
}

\author{
Alexei Tchistyi \\ NYU Stern School of Business \\ atchisty@stern.nyu.edu
}

October 3, 2005

\begin{abstract}
This paper studies optimal security design in a dynamic setting with an agency problem that arises when an agent in charge of a project can divert cash flows for his own consumption at the expense of an outside investor. Cash flows are unobservable and unverifiable by the outside investor, who relies on the agent's reports, and has the right to liquidate the project. Unlike previous analyses, we allow cash flows to be correlated over time. We solve for the optimal contract and show that it can be implemented using a credit line with an interest rate that increases with the balance on the credit line. This finding is consistent with the fact that the majority of commercial loans are lines of credit with performance pricing. Thus, our model provides theoretical evidence that performance pricing is used to mitigate the agency cost. In addition, we develop a new recursive method to deal with a correlated privately observed variable in dynamic agency settings. It allows us to reduce the dimensionality of the problem and obtain a closed-form solution for the optimal contract.
\end{abstract}

\footnotetext{
${ }^{*}$ I am extremely grateful to Peter DeMarzo for advice throughout the development of the paper. I also thank Manuel A. Amador, Darrell Duffie, Gustavo Manso, Yuliy Sannikov, Ilya Segal, Ilya Strebulaev, Andrzej Skrzypacz, Bruno Strulovici, and Robert Wilson for helpful comments and discussions. I thank Stanford Institute for Economic Policy Research for financial support through Lynde and Harry Bradley Foundation Dissertation Fellowship.
} 


\section{Introduction}

More than $87 \%$ of all commercial and industrial loans made by large domestic banks are loans under commitment ${ }^{1}$, otherwise known as lines of credit. A line of credit is a contract between a firm and a bank that lets the firm borrow from the bank during the life of the contract on terms specified in advance. Two main characteristics of a credit line are the credit limit, which stipulates the maximum amount of credit allowed, and the interest rate charged on the balance. Very often, instead of a fixed rate of interest, performance pricing schemes are used that connect the interest rate to some measure of the borrower's performance, such as the borrower's interest coverage ratio, debt-to-EBITDA ratio, leverage ratio, or current credit rating. Asquith, Beatty and Weber (2004) report that more than $50 \%$ of lending agreements $^{2}$ have performance pricing features. Moreover, most of the lending agreements require the borrower to pay a higher interest rate when the borrower performs poorly.

Manso, Strulovici and Tchistyi (2004) study different forms of performance pricing in a dynamic capital-structure model, in which default is endogenously triggered by equity holders when the firm's assets fall to a certain level. In a setting with a bankruptcy cost and tax benefits but without an agency problem, they prove that debt obligations that result in paying higher interest rates in times of poor performance and lower interest rates in times of high performance are not the most efficient way to raise money. Such debt obligations precipitate default and increase bankruptcy cost, since they impose a higher debt burden when the firm experiences financial strains. A loan with a fixed interest rate results in a higher value of the firm. This finding raises a question: why are such performance pricing schemes so widely used in practice?

The goal of this paper is to explain the existence of the very popular form of bank lending: a line of credit with an escalating interest rate. We develop a model of security design in which a line of credit with an escalating interest rate is a part of the optimal contract. In the friction-free world of Modigliani and Miller (1958), the market value of the firm is independent of its capital structure. As Manso, Strulovici and Tchistyi (2004) establish, the theory of trade-off between tax benefits and bankruptcy costs does not justify performance pricing. This paper introduces an agency problem into the model and shows that performance pricing is used to mitigate the agency cost.

Specifically, a risk-neutral agent with limited liability needs external financing for a profitable business project. If funded, the project generates stochastic cash flows. An outside investor is unable to observe the cash flows, while the agent has an ability to divert the cash flows for his own consumption at the expense of the investor. Before initiating the project, the agent and the investor (or a group of investors) sign a contract that will govern their

\footnotetext{
${ }^{1}$ E.2 Survey of Terms of Business Lending, August 2-6, 2004 (Federal Reserve Statistical Release)

${ }^{2}$ Asquith, Beatty and Weber (2004) use the data on loan contracts in the Loan Pricing Corporation database.
} 
relationship after the project is initiated. In particular, the contract obligates the agent to report the cash flows to the investor, although the investor cannot verify the agent's reports. In addition, the contract specifies payments between the agent and the investor conditional on the history of the agent's reports, and the circumstances under which the control of the project's assets is transferred from the agent to the investor. In this latter case, the agent is fired and the investor makes optimal use of the assets. The transfer of control leads to inefficiencies, either due to a dead-weight cost associated with it, or because the investor is less capable than the agent of running the project and cannot find an equivalent replacement for the agent's managerial talent.

We assume that the cash flows are correlated over time and follow a two-state Markov process. The correlation is an important assumption, not only because it is a more realistic assumption than independent cash flows, but also because it creates an additional degree of informational asymmetry between the agent and the investor. With correlated cash flows, the agent has a superior knowledge, not only about the current cash flow realization that he observes directly, but also about the future cash flows, since their distribution is determined by the current cash flow realization.

We characterize the optimal contract in this setting and its implementation using standard securities. We find that the optimal contract can be implemented using a combination of equity, a coupon bond and a credit line with an interest rate that increases with the outstanding balance on the credit line. According to this implementation, the agent owns a fraction of the firm's equity, while being obligated to make coupon payments on the bond and interest payments on the credit line balance to the investor, who also owns the rest of the firm's equity. The initial draw on the credit line is determined by the amount of funds provided by the investor, as well as the bargaining power of the parties. The agent uses the cash flows generated by the project to make the current debt payments and to repay the balance on the credit line. When the cash flow is low, the agent is allowed to draw on the credit line to make the current debt payments, as long as he does not exceed the credit limit. The agent is in default if he is unable to fulfill his current debt obligations without exceeding the credit line limit. In this case, the investor takes control over the firm's assets and fires the agent.

In this combination of securities, the roles of the coupon bond and equity are straightforward. The coupon bond is used to extract verifiable cash flows, while dividends paid to the equity holders offer a reward to the agent for repaying the debt. The role of the credit line with an escalating interest rate is more sophisticated. The balance on the credit line can be considered as a memory device that summarizes all the relevant information regarding the agent's performance. The interest rate, along with the credit limit, determines the dynamics of the credit line balance and the timing of the default. When the agent reports a low cash flow and fails to make the interest payment, the balance on the credit line increases by the 
amount of the unmade interest payment. When the balance goes over the credit limit default occurs. The speed at which the balance grows is determined by the interest rate; and this speed is greater when the balance is higher. The agent has the incentive to use high cash flows to reduce the balance as well as the interest rate. The threat of losing control over the project induces the agent to pay the debt.

To see why the interest rate on the credit line should increase with the balance, consider what will happen when the agent keeps stealing the cash flows until the credit line is exhausted and default occurs. Because the cash flows are positively correlated, the average amount that can be stolen per period is negatively related to the length of the time interval during which the stealing takes place. Higher balance on the credit line means that the default will occur sooner if the agent keep stealing cash flows. Thus, to discourage the agent from stealing, a higher interest rate should be charged on the credit line when the balance is high.

An important aspect of this paper is the methodology, which has independent theoretical value. We develop a new dynamic programming approach for solving for an optimal contract (mechanism) in a setting with a correlated privately observed variable, where the standard dynamic programming technique does not work. The main advantage of our approach is that it allows us to reduce the dimensionality of the problem and obtain a closed-form solution for the optimal contract in our setting. We also believe that this approach is not only applicable to our setting, but can also be used in other dynamic principal-agent models with correlated hidden states.

\section{$1.1 \quad$ Related Literature}

A number of papers study optimal contracting in a setting in which an agent has an ability to divert cash flows. In a simple one-period model, Diamond (1984) demonstrates that the optimal contract is debt, where the agent's incentives to make payments to lenders are given in terms of non-pecuniary bankruptcy penalties. Bolton and Scharfstein (1990) consider a similar two-period model, in which the investor can threaten to cut off funding in the second period if the firm defaults in the first. This threat induces the firm to share the first period profit with the investor. In a dynamic setting with asymmetric information, Clementi and Hopenhayn (2004) demonstrate that borrowing constraints emerge as a feature of the optimal lending agreements.

The two studies that are most closely related to ours are DeMarzo and Fishman (2003), and DeMarzo and Sannikov (2004). Both of these papers study long-term financial contracting in a setting with privately observed independent cash flows. Unlike previous analyses, we allow cash flows to be correlated over time. It turns out that the correlation significantly changes the optimal contract between the agent and the investor. While in DeMarzo and Fishman (2003), and DeMarzo and Sannikov (2004) the optimal contract can be implemented 
using a credit line with a constant interest rate, we find that the implementation of the optimal contract in our setting requires a credit line from the investor with a variable interest rate. Since the presence of correlation of cash flows is overwhelming in practice, our model is a more realistic approximation of the reality, and should better fit the data.

Recent empirical studies support the theory that performance pricing and other debt covenants are used to mitigate agency costs. Analyzing a large database of commercial loans, Asquith, Beatty and Weber (2004) report that debt contracts are more likely to include performance pricing when re-contracting, adverse selection, and moral hazard costs are higher. They also estimate that more than $50 \%$ of debt contracts have performance pricing requirements. While using the same database, Bradley and Roberts (2004) come to the conclusion that debt covenants are used to reduce the agency cost of debt. Dichev and Skinner (2004) relate the existence of debt covenants to informational asymmetries between lenders and borrowers, which may cause agency problems.

On the technical side, this paper develops a recursive method to solve for an optimal incentive-compatible contract in a setting in which a privately observed state variable is correlated over time. The vast majority of the literature on optimal contracting assumes, however, that privately observed economic variables are independent over time. In this literature, an optimal contract typically depends on a history of publicly observed outcomes, which is a multi-dimensional object. However, it is often possible to rewrite the problem recursively, summarizing all the relevant information in the history by a one-dimensional object - a continuation value. Green (1987), Abreu, Pearce, and Stacchetti (1990), Phelan and Townsend (1991), Korcherlakota (1996), DeMarzo and Fishman (2002), DeMarzo and Fishman (2003), among many others, utilize this approach.

Surprisingly, there are only a few papers that allow for correlation of privately observed variables. Fernandes and Phelan (2000) consider a dynamic model with a risk-averse agent whose endowment follows a first-order Markov process. Quadrini (2003) uses the methodology developed by Fernandes and Phelan (2000) to solve a dynamic principal-agent model with privately observed persistent shocks. Doepke and Townsend (2001) develop several new recursive methods to solve for optimal contracts in dynamic principal-agent models with hidden income and hidden actions. These papers resort to numerical simulations to characterize optimal contracts.

Our approach to solving an agency problem with history dependence is quite different from the methodology of Fernandes and Phelan (2000). Their method requires computation of the continuation functions that depend, not only on the state variable and the agent's continuation payoff, but also on the agent's deviation payoff. Although their method can be used in our setting for numerical simulations, the higher dimensionality of their method makes it virtually intractable analytically.

Battaglini (2004) obtains a closed form solution for the optimal contract between a mo- 
nopolist and a consumer whose preferences follow a two-state Markov process and are unobservable by the monopolist. The consumer has unlimited wealth and the consumer's type at time zero is unknown to the monopolist. According to the optimal contract, the monopolist screens the consumer's type by distorting consumption of the low type. The contract instantly becomes efficient as soon as the consumer reveals his type is high. In a related paper, Battaglini and Coate (2004) study optimal income taxation of individuals whose income generating abilities evolve according to a two-state Markov process. The major distinction of our paper from Battaglini (2004), and Battaglini and Coate (2004) is that the agent has limited wealth in our setting. As a result, the first best cannot be implemented even when the cash flow is high. Consequently, the structure of the optimal contract in our setting is quite different from that in Battaglini (2004), and Battaglini and Coate (2004). In particular, in their papers, the optimal contract depends only on whether the agent has ever reported the high type, at which point the contract becomes first best. Here, the contract remains second best even after multiple reports of the high cash flows, and the optimal contract depends on history through a continuous variable, the balance on the credit line.

The paper is organized as follows. Section 2 introduces the dynamic contracting model with correlated privately-observed cash flows. Section 3 contains a preliminary analysis of the contracting problem. Section 4 provides the derivation of the optimal contract. Section 5 demonstrates that the optimal contract can be implemented by a combination of equity, a coupon bond and a credit line with an escalating interest rate. Section 6 extends the model to the continuous time setting. Section 7 concludes.

\section{The Model}

A risk-neutral agent evaluates consumption sequences $\left\{C_{t}\right\}$ according to $\sum_{t} \beta^{t} E\left[C_{t}\right]$, where $\beta$ is the intertemporal discount factor. The agent has a special human capital to run a project that generates stochastic cash flows $\left\{Y_{t}\right\}$. The agent's initial wealth $W$ is not sufficient to initiate the project that requires a fixed initial investment $I>W$. To raise the lacking capital, the agent will have to enter into a contractual relationship with an investor who is also risk-neutral and has sufficient financial resources. The discount factor for the investor is also $\beta$, which corresponds to the risk-free interest rate $r=\frac{1}{\beta}-1$.

The cash flows generated by the project are correlated over time. For simplicity, let the cash flows follow a two-state Markov chain: $Y_{t} \in\left\{y_{L}, y_{H}\right\}$ for all $t$, where $0 \leq y_{L}<y_{H}$. We

will refer to $y_{L}$ and $y_{H}$ as the low and the high cash flows respectively. Let $Q(y)$ denote the probability that $Y_{t}=y_{H}$, given the previous period cash flow realization $y \in\left\{y_{L}, y_{H}\right\}$ :

$$
Q(y)=\operatorname{Pr}\left(Y_{t}=y_{H} \mid Y_{t-1}=y\right) .
$$


The cash flows are assumed to be positively correlated, which in terms of transition probabilities means that $Q\left(y_{H}\right)>Q\left(y_{L}\right)$. One can verify that this implies that an expectation of a future cash flow is always higher in the high state:

$$
E\left[Y_{t+k} \mid Y_{t}=y_{H}\right]>E\left[Y_{t+k} \mid Y_{t}=y_{L}\right]
$$

where $k=1,2, \ldots$ For more on the properties of the cash flow process, see Lemma 5 in Appendix.

The agent privately observes realizations of the cash flows, while the investor must rely on the agent to report the cash flow realizations, without being able to verify the agent's reports. We assume that the low cash flow $y_{L}$ is observable and collectible by the investor, but the agent can secretly divert the excess cash flow $y_{H}-y_{L}$ for his own consumption. Cash flow diversion may be costly for the agent. It is assumed that the agent is able to enjoy only a fraction $\lambda \in[0,1]$ of the diverted amount. The fraction $(1-\lambda)$ represents the cost of stealing, which can be attributed to different kinds of expenses and inefficiencies associated with the conspiracy. The agent can consume diverted cash flows immediately or save them at the interest rate $\rho \leq r$ at his private bank account.

We interpret diversion of the firm's cash flows as stealing. We assume that the agent can secretly transfer money from the firm's account to his own account using accounting manipulations. However, other activities that benefit the agent at the expense of the investor and are not observable by the investor may fit the setting of the model as well. For example, the agent can inefficiently use the firm's cash flows in order to receive non monetary benefits, such as corporate jets. The fact that the agent cannot save non-monitary benefits is not essential here, since, as we will see it later, savings cannot improve the agent's payoff anyway.

The agent has limited liability: he can quit at any time and get his reservation payoff $R_{t}$. For ease of presentation, we normalize the agent's reservation payoff to zero: $R_{t}=0$, which means that the agent will never quit voluntarily. In addition, it is assumed, for technical reasons, that the project has a finite life, with $T$ being the final date.

In exchange for the funding, the investor gains the right to take control over the project. We refer to it as the liquidation of the project. If the project is liquidated at date $t$, the investor sells the project's assets and collects the liquidation value $L_{t}$, while the agent is left with his reservation value $R_{t}$. All the subsequent cash flows are permanently lost. We assume that the liquidation is inefficient, in the sense that the liquidation value is strictly below the expected value of the cash flows lost in the case of liquidation. The inefficiency may be caused by friction costs associated with a transfer of control, or by the fact that the agent's unique human capital is required to run the project efficiently. We assume that the 


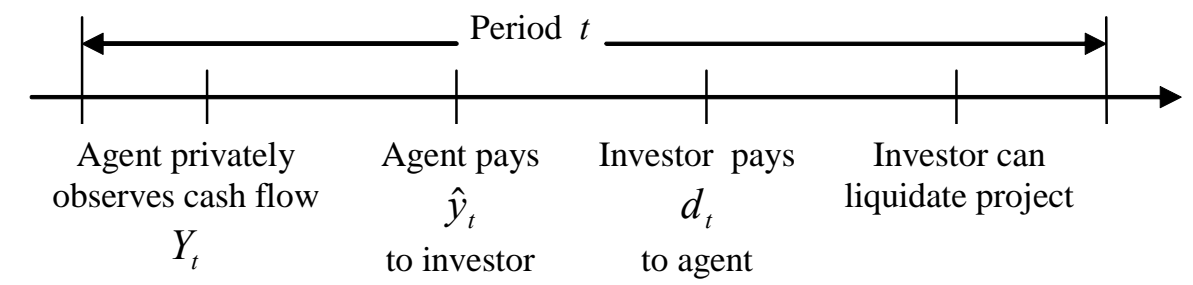

Figure 1: Sequence of Events

value of the liquidated firm in period $t$ is given by

$$
L_{t}\left(y_{t}\right)=\sum_{k=t+1}^{T} \beta^{k-t} y_{L}+\alpha \sum_{k=t+1}^{T} \beta^{k-t} E\left[Y_{k}-y_{L} \mid Y_{t}=y_{t}\right],
$$

where $\alpha \leq 1$. According to (2), the investor is able to recover the full value of the future minimum cash flows and the fraction $\alpha$ of the value of the future excess cash flows. The liquidation is strictly inefficient when $\alpha<1$.

If the investor agrees to fund the project, at date 0 the agent and the investor sign a contract that will govern their relationship until the final date $T$. According to the contract, the agent must report realizations of the cash flows to the investor. Of course, the reported cash flow $\hat{y}_{t}$ can be different from the true realization $y_{t}$. Without loss of generality, we assume that the contract requires that the agent pays the reported cash flows to the investor immediately. The contract also specifies transfer payment $d_{t}$ to the agent and the probability of liquidation $p_{t}$ after any history of the agent's reports. Specifically, given the history $\hat{y}^{t}=\left\{\hat{y}_{1}, \ldots, \hat{y}_{t}\right\}$ of the agent's reports, the contract obligates the investor to make a payment of $d_{t}\left(\hat{y}^{t}\right) \geq 0$ to the agent, and liquidate the project with the probability $p_{t}\left(\hat{y}^{t}\right)$ at the end of period $t$. The sequence of the events is illustrated in Figure 1.

We will study contracts with full commitment. No renegotiation of the terms of the contract is allowed. A contract is optimal if it maximizes the investor's continuation payoff subject to a certain payoff for the agent.

\section{Preliminary Analysis of the Dynamic Contracting Problem}

In order to solve for the optimal contract between the agent and the investor, we need to find the transfers $d_{t}\left(\hat{y}^{t}\right)$ and the probabilities of liquidation $p_{t}\left(\hat{y}^{t}\right)$ for each possible history of the reports $\hat{y}^{t}$ at each time $t$ that maximize the expected payoff for the investor at time zero, given the expected payoff $a_{0}$ for the agent. Since the number of possible histories double 
each period, the dimensionality of the problem increases exponentially with $T$. In order to deal with the high dimensionality of the problem, we have to rewrite the problem recursively. We start our analysis of the contracting problem by showing that the optimal contract can be implemented using a direct revelation mechanism without savings. We then show that the optimal contract is incentive compatible if and only if there is no profitable one-period deviation from the truth telling strategy.

\subsection{Optimality of a Truth-Telling Contract without Savings}

We start our analysis of the model by showing that we can restrict our attention to the set of contracts in which the agent always tells the truth and does not save.

For any contract $\sigma=(d, p)$, the agent chooses an optimal strategy $\varphi=(\hat{y}, C, S)$ that, apart from the reporting strategy $\hat{y}$, also includes the agent's consumption $C$, and saving $S$, as functions of the history $y^{t}$ of the cash flow realizations. At every date $t$, the agent's consumption $C_{t}$, and the savings $S_{t}$ must be non-negative. The agent's income

$$
i_{t}=d_{t}+\zeta\left(y_{t}, \hat{y}_{t}\right)
$$

consists of two components: the transfer $d_{t}$ from the investor and the difference $\zeta\left(y_{t}, \hat{y}_{t}\right)$ between the reported cash flow $\hat{y}_{t}$ and the actual cash flow realization $y_{t}$ :

$$
\zeta\left(y_{t}, \hat{y}_{t}\right)=\lambda\left(y_{t}-\hat{y}_{t}\right)^{+}-\left(y_{t}-\hat{y}_{t}\right)^{-} \text {. }
$$

Note, that $\zeta\left(y_{t}, \hat{y}_{t}\right)$ can be negative, since the agent can use his savings to overreport cash flows.

One can use a Revelation-Principle type of logic to show that, for any contract $\sigma=(d, p)$, there exists a contract $\sigma^{\prime}=\left(d^{\prime}, p^{\prime}\right)$ that results in the same payoff for the agent and equal or greater payoff for the investor, and for which truth-telling is the optimal strategy for the agent. Indeed, suppose the reporting strategy $\tilde{y}$ is optimal under the contract $\sigma$, then define $d_{t}^{\prime}\left(\hat{y}^{t}\right)=d_{t}\left(\tilde{y}_{t}\left(\hat{y}^{t}\right)\right)+\zeta\left(\hat{y}_{t}, \tilde{y}_{t}\left(\hat{y}^{t}\right)\right)$, and $p_{t}^{\prime}\left(\hat{y}^{t}\right)=p_{t}\left(\tilde{y}_{t}\left(\hat{y}^{t}\right)\right)$, for every history $\hat{y}^{t}$ of the agent's reports. One can see that if the agent tells the truth under $\sigma^{\prime}$, then his income in each period is equal to the income he receives under $\sigma$ when he employs strategy $\tilde{y}$; and the investor's payoff under $\sigma^{\prime}$ is no less than under the old contract ${ }^{3}$. In addition, the truth-telling is optimal under $\sigma^{\prime}$, since $\tilde{y}$ is the optimal strategy under $\sigma$.

Savings are not necessary for the agent because the agent is risk-neutral. Since the agent finds it optimal to tell the truth under the contract $\sigma^{\prime}$, he never uses his savings to misrepresent cash flows. Thus, savings translate into delayed consumption. Since the agent

\footnotetext{
${ }^{3}$ Since fraction $(1-\lambda)$ of the diverted cash flows is wasted, the investor's income under the contract $\sigma^{\prime}$ can be actually higher than that under the contract $\sigma$, since the agent never diverts cash flows under the contract $\sigma$.
} 
is risk-neutral, he receives no benefits from consumption smoothing.

This leads to the following result:

Proposition 1 There exists an optimal contract that induces the agent to report cash flows truthfully and maintain zero savings.

\subsection{Temporary Incentive Compatibility Constraints}

Given the result of Proposition 1, we will focus on direct-revelation contracts with no savings for the agent. To facilitate our analysis, we assume that the agent is not allowed to save, which implies that the agent cannot report $y_{H}$ when $y_{L}$ is realized. After finding an optimal contract without savings, we will verify that the contract remains incentive compatible when the agent is allowed to save. We say that the reporting strategy $\hat{y}$ is feasible if and only if $\hat{y}_{t} \leq y_{t}$, where $\hat{y}_{t} \in\left\{y_{L}, y_{H}\right\} .4$

First, we prove that the contract is incentive compatible if and only if at any point of time the agent's continuation payoffs satisfy temporary incentive compatibility constraints.

The agent's reporting strategy $\hat{y}$ is a sequence $\left\{\hat{y}_{t}\left(y^{t}\right)\right\}_{t=1}^{T}$ of the agent's reports about the cash flow realizations. If the agent reports $\hat{y}_{t}$, given the actual cash flow $y_{t}$, his net income in period $t$ is given by $y_{t}-\hat{y}_{t}+d_{t}\left(\hat{y}^{t}\right)$. Let $P_{t}\left(\hat{y}^{t}\right)$ be the probability that the project is active at the beginning of period $t$ after the history $\hat{y}^{t}$ of reports, under the contract $\sigma=(d, p)$.

One can verify that $P_{t}\left(\hat{y}^{t}\right)=\prod_{k=1}^{t-1}\left(1-p_{t}\left(\hat{y}^{k}\right)\right)$. The reporting strategy $\hat{y}$ under the contract $\sigma$ results in the following expected payoff for the agent:

$$
a_{0}\left(y_{0}, \hat{y}, \sigma\right)=E\left[\sum_{t=1}^{T} \beta^{t} P_{t}\left(\hat{y}^{t}\right)\left(\lambda\left(Y_{t}-\hat{y}_{t}\right)+d_{t}\left(\hat{y}^{t}\right)\right) \mid Y_{0}=y_{0}\right] .
$$

Note that the agent's payoff also depends on the initial state $y_{0}$.

We say that a contract $\sigma=(d, p)$ is incentive compatible if it induces the agent never to misreport the cash flows. That is, for any reporting strategies $\hat{y}$,

$$
E\left[\sum_{t=1}^{T} \beta^{t} P_{t}\left(y^{t}\right) d_{t}\left(y^{t}\right) \mid Y_{0}=y_{0}\right] \geq E\left[\sum_{t=1}^{T} \beta^{t} P_{t}\left(\hat{y}^{t}\right)\left(\lambda\left(Y_{t}-\hat{y}_{t}\right)+d_{t}\left(\hat{y}^{t}\right)\right) \mid Y_{0}=y_{0}\right]
$$

Under an incentive compatible contract, the agent's best strategy is to truthfully reveal the cash flows in every period. Will the truth-telling strategy remain optimal on an off-

\footnotetext{
${ }^{4}$ The agent can, of course, report a cash flow $\hat{y}$, which is greater than $y_{L}$, but less than $y_{H}$. Such a reporting strategy cannot be optimal for the agent under the optimal contract, since it would immediately reveal that the true cash flow was $y_{H}$ and the agent stole $\left(y_{H}-\hat{y}\right)$. Under the optimal contract, the investor commits to punish the agent for stealing by liquidating the project immediately if the agent reports anything other than $y_{L}$ or $y_{H}$. Hence, the agent strictly prefers to report $y_{L}$ over $\hat{y} \in\left(y_{L}, y_{H}\right)$.
} 
equilibrium path? The next result shows that an incentive compatible contract remains incentive compatible even if the agent has deviated from truth-telling in the past.

Lemma 1 Under any incentive compatible contract, at any point of time, the agent (weakly) prefers to tell the truth, even if he has lied in the past.

Proof. See Appendix.

After a realization of cash flow $y_{H}$ the agent has a dilemma. He can either tell the truth and report the high cash flow, or report the low cash flow and consume the stolen $\lambda\left(y_{H}-y_{L}\right)$. Reporting $y_{H}$ truthfully means choosing a continuation contract that follows after the report $y_{H}$, while reporting $y_{L}$ and diverting $y_{H}-y_{L}$ means choosing a continuation contract that follows after the report $y_{L}$. Let the continuation contract $\sigma_{t}\left(\hat{y}^{t}\right)$ correspond to the history $\hat{y}^{t}$ of the agent's reports. The terms of this contract depend on the reported cash flows, but not on the actual realizations of the cash flows. However, the last cash flow realization matters for the agent's and the investor's continuation payoffs, because it determines the distribution of the future cash flows.

Let's consider a one-time deviation from the truth-telling strategy. Suppose $Y_{t}=y_{H}$. Given a history $y^{t}$ of the cash flows realizations, such that the project can be active in period $t$, i.e. $P_{t}\left(y^{t}\right)>0$, the agent's continuation payoff under the truth-telling strategy is given by

$$
a_{t}\left(y^{t-1}, y_{H}\right)=E\left[\sum_{k=t}^{T} \beta^{k-t} \frac{P_{k}\left(y^{t}, y^{k-t}\right)}{P_{t}\left(y^{t}\right)} d_{t}\left(y^{t}, y^{k-t}\right) \mid Y_{t}=y_{H}\right] .
$$

If the agent truthfully reveals the cash flows in each period other than $t$, but cheats in period $t$, the agent's continuation payoff will be

$$
\hat{a}_{t}\left(y^{t-1}, y_{H}\right)=E\left[\sum_{k=t}^{T} \beta^{k-t} \frac{P_{k}\left(y^{t-1}, y_{L}, y^{k-t}\right)}{P_{t}\left(y^{t-1}, y_{L}\right)} d_{t}\left(y^{t-1}, y_{L}, y^{k-t}\right) \mid Y_{t}=y_{H}\right]
$$

We will call $\hat{a}_{t}\left(y^{t-1}, y_{H}\right)$ the deviation continuation payoff after history $\left(y^{t-1}, y_{H}\right)$.

The next theorem says that the contract is incentive compatible if and only if there is no one-time profitable deviation for the agent.

Theorem 1 The contract $\sigma=(d, p)$ is incentive compatible if and only if for all time periods $t \leq T$, and all histories $y^{t-1}$, such that $P_{k}\left(y^{t-1}\right)>0$,

$$
a_{t}\left(y^{t-1}, y_{H}\right) \geq \hat{a}_{t}\left(y^{t-1}, y_{H}\right)+\lambda\left(y_{H}-y_{L}\right)
$$

Proof. See Appendix.

Equation (5) says that, under the contract $\sigma$, the agent does not find it optimal to deviate from the truth-telling strategy for one period. We will interpret equation (5) as 
the temporary incentive compatibility constraint. Theorem 1 establishes that a contract is incentive compatible if and only if at any point of time temporary incentive compatibility constraints are satisfied. We will use this property later when we formulate the contracting problem recursively.

\subsection{Contracting Problem}

The investor's income in each period is given by the difference between the reported cash flow $\hat{y}_{t}$ and the payment to the agent $d_{t}$ and the proceeds $L_{t}$ from the asset liquidation. Given the initial state $y_{0}$ and the continuation payoff $a_{0}$ for the agent, the investor's problem is to choose an incentive compatible contract $\sigma=(d, p)$ that maximizes the investor's payoff:

$$
b_{0}\left(y_{0}, a_{0}\right)=\max _{d, p} E_{0}\left[\sum_{t=1}^{T} \beta^{t} P_{t}\left(y^{t}\right)\left(Y_{t}-d_{t}\left(y^{t}\right)+p_{t}\left(y^{t}\right) L_{t}\right) \mid Y_{0}=y_{0}\right],
$$

subject to the incentive compatibility constraint

$$
E\left[\sum_{t=1}^{T} \beta^{t} P_{t}\left(y^{t}\right) d_{t}\left(y^{t}\right) \mid Y_{0}=y_{0}\right] \geq E\left[\sum_{t=1}^{T} \beta^{t} P_{t}\left(\hat{y}^{t}\right)\left(\lambda\left(Y_{t}-\hat{y}_{t}\right)+d_{t}\left(\hat{y}^{t}\right)\right) \mid Y_{0}=y_{0}\right],
$$

for all feasible reporting strategies $\hat{y}$, and the promise keeping constraint

$$
a_{0}=E_{0}\left[\sum_{t=1}^{T} \beta^{t} P_{t}\left(y^{t}\right) d_{t}\left(y^{t}\right) \mid Y_{0}\right]
$$

where $y^{t}=\left\{y_{1}, \ldots y_{t}\right\}$ denotes the history of the cash flow realizations.

An optimal contract solves the investor's problem given by (6)-(8). Any other contract results in a payoff for the investor being equal to or lower than $b_{0}\left(y_{0}, a_{0}\right)$, given the agent's payoff $a_{0}$. Thus, function $b_{0}\left(y_{0}, a_{0}\right)$ represents the highest possible payoff attainable by the investor, given the payoff $a_{0}$ for the agent and the initial state $y_{0}$.

Solving for the contracting problem (6)-(8) means finding the optimal transfers $d_{t}\left(\hat{y}^{t}\right)$

and the optimal probabilities of liquidation $p_{t}\left(\hat{y}^{t}\right)$ for each possible history $\hat{y}^{t}$ of the reports at each time $t$. Since the number of possible histories double each period, the number of unknown variables in the problem is given by $2\left(2^{T}-1\right)$. In order to deal with the high dimensionality of the problem, we have to rewrite the problem recursively.

\subsection{Recursive Formulation of the Contracting Problem}

The setting of our model is similar to DeMarzo and Fishman (2003). However, unlike previous analyses, we allow the cash flows to be positively correlated over time. This is an important assumption not only because it is a more realistic assumption than independent cash flows, but also because the correlation creates an additional degree of informational asymmetry 
between the agent and the investor. With the correlated cash flows, the agent has a superior knowledge not only about the current cash flow realization that he observes directly, but also about the future cash flows, since their distribution is determined by the current cash flow. By misrepresenting the current cash flow the agent also misrepresents the true quality of the project.

The correlation assumption is also a costly assumption. The vast majority of literature on dynamic contracting assumes that privately observed variables are independent over time. This gets us common knowledge of preferences over continuation contracts. With the correlated cash flows, preferences over continuation contracts are no longer common knowledge. Therefore, standard techniques of dynamic programming do not work, and we need to develop our own techniques in order to solve the contracting problem.

\subsubsection{Recursive Formulation of the Contracting Problem with Independent Cash Flows}

Before approaching the contracting problem with the correlated cash flows, it may be useful to see how standard techniques of dynamic programming can be applied in the case with independent cash flows, and why these techniques do not work with correlated cash flows.

When the cash flows are independent over time, at any point of time continuation payoffs for the agent and the investor associated with the continuation contracts do not depend on the current cash flow realization. Since the investor has the complete knowledge of the agent's preferences over continuation contracts one can formulate the dynamic contracting problem recursively using the continuation payoff for the agent as a state variable. The key element of the recursive formulation is the way temporary incentive compatibility constraints are written. Let $a_{t}^{d}\left(y_{L}\right)$ and $a_{t}^{d}\left(y_{H}\right)$ denote payoffs for the agent under the optimal continuation contracts after the agent reports the low and the high cash flow respectively. The agent truthfully reveals the cash flow realization in period $t$ if

$$
a_{t}^{d}\left(y_{H}\right) \geq a_{t}^{d}\left(y_{L}\right)+\lambda\left(y_{H}-y_{L}\right) \text {. }
$$

Given incentive compatibility constraint (9), one can compute the optimal contract by backward induction, using the continuation payoff $a_{t}^{d}$ as a single state variable. According to the recursive procedure used by DeMarzo and Fishman (2003), the investor maximizes his continuation payoff in each period by choosing continuation payoffs for the agent subject to the incentive compatibility constraints. Each continuation payoff for the agent uniquely determines the optimal continuation contract. Thus, choosing continuation payoffs for the agent is equivalent to choosing optimal continuation contracts. Since the optimal contract is computed by backward induction starting from the last period for each possible continuation payoff for the agent, one can use the agent's continuation payoff $a_{t}^{d}$ as a state variable in 
order to formulate the problem recursively.

\subsubsection{Recursive Formulation of the Contracting Problem with Markov Cash Flows}

The above approach is not applicable in the setting with correlated cash flows since the incentive compatibility constraint (9) is no longer true. Figure 2 demonstrates how the incentive compatibility conditions are different with the Markov cash flows. Suppose the expected payoff for the agent at the beginning of period $t$ is $a_{t}^{y}$. In the i.i.d. case, in order to induce the agent to reveal the cash flow realization truthfully, the agent's continuation payoff $a_{t}^{d}\left(y_{H}\right)$ after he reports the high cash flow should be higher than $a_{t}^{y}$ and the agent's continuation payoff $a_{t}^{d}\left(y_{L}\right)$ after he reports the low cash flow should be lower than $a_{t}^{y}$, so that IC constraint (9) holds. However, when the cash flows are positively correlated over time, a continuation contract that results in the continuation payoff $a_{t}^{d}\left(y_{L}\right)$ for the agent in the low state will result in the agent's payoff $\hat{a}_{t}^{d}\left(y_{L}\right)$, if the agent reports the low cash flow when the high cash flow is actually realized. Since the future cash flows are more likely to be high when the current state is high, $\hat{a}_{t}^{d}\left(y_{L}\right)>a_{t}^{d}\left(y_{L}\right)$. Thus the incentive compatibility constraint with the Markov cash flows can be written as

$$
a_{t}^{d}\left(y_{H}\right) \geq \hat{a}_{t}^{d}\left(y_{L}\right)+\lambda\left(y_{H}-y_{L}\right)
$$

While $a_{t}^{d}\left(y_{L}\right)$ represents the agent's continuation payoff in equilibrium, $\hat{a}_{t}^{d}\left(y_{L}\right)$ is the agent's continuation payoff in deviation. Fernandes and Phelan (2000) use both the continuation payoff in equilibrium and the continuation payoff in deviation as state variables to formulate their contracting problem recursively. However, this method requires computation of the continuation functions that depend on the two state variables. Although their method can be used in our setting for numerical simulations, the higher dimensionality of their method makes it virtually intractable analytically.

Our approach to solving the contracting problem is to formulate it recursively using the agent's continuation payoff in equilibrium as a single state variable. We will do so by finding

the relationship between the agent's continuation payoff in the low state $a_{t}^{d}\left(y_{L}\right)$ and the agent's continuation payoff in deviation $\hat{a}_{t}^{d}\left(y_{L}\right)$. Both $a_{t}^{d}\left(y_{L}\right)$ and $\hat{a}_{t}^{d}\left(y_{L}\right)$ are related to the same continuation contract. The only difference between these two payoffs is that the first payoff is obtained using the distribution of the future cash flows conditional on the low state, while the second payoff is obtained using the distribution of the future cash flows conditional on the high state. Using backward induction, we find function $c_{t}$ that relates these two payoffs:

$$
\hat{a}_{t}^{d}\left(y_{L}\right)=c_{t}\left(a_{t}^{d}\left(y_{L}\right)\right)
$$




\section{i.i.d. cash flows}

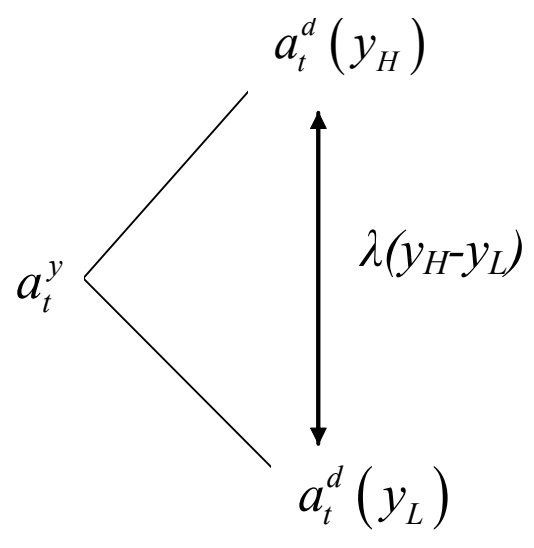

Markov cash flows

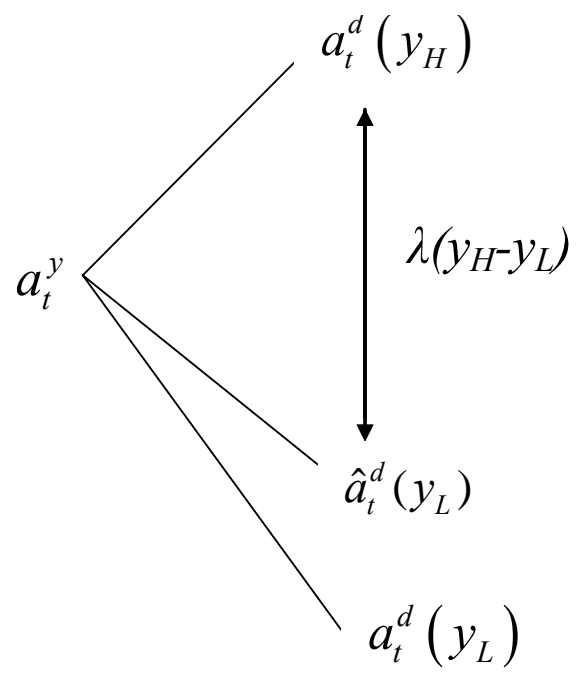

Figure 2: Incentive compatibility with independent and Markov cash flows.

Function $c_{t}$ is monotonic in its argument, which is not surprising: an optimal continuation contract that yields higher payoff for the agent in the low state should also yield a higher payoff in the high state. Using function $c_{t}$ we can write down the incentive compatibility constraint with the Markov cash flows as follows:

$$
a_{t}^{d}\left(y_{H}\right) \geq c_{t}\left(a_{t}^{d}\left(y_{L}\right)\right)+\lambda\left(y_{H}-y_{L}\right)
$$

The main difference between (10) and (11) is that (11) depends only on the equilibrium payoff for the agent, while (10) depends on both equilibrium and deviation payoffs. When function $c_{t}$ is an identity, equation (11) is equivalent to (9). Thus, the IC constraint with independent cash flows (9) is a special case of the IC constraint with Markov cash flows(11). Exploiting the similarities between (11) and (9), in particular the fact that the IC constraint (11) is expressed in terms of the equilibrium payoffs only, we will adopt the dynamic programming techniques with independent cash flows to the Markov case in Section 4.

\section{The Optimal Contract}

In this section, we solve for the optimal contract. First, in order to rewrite the contracting problem recursively, we conjecture that the optimal contract is sequentially optimal. Second, given the conjecture, we solve for the optimal contract using a dynamic programming technique. A key ingredient of our technique is that, in order to write down temporary incentive 
compatibility constraints, we calculate the agent's continuation payoffs in the high state as a function of the agent's continuation payoffs in the low state under the optimal contract. Third, we verify that the contract we derive under the sequential optimality conjecture is indeed the best possible contract. Finally, we talk about initiating the optimal contract.

\subsection{Sequential Optimality Conjecture}

Recall the definition of an optimal contract. We say that an incentive-compatible contract $\sigma$ that implements payoffs $a_{0}\left(y_{0} \mid \sigma\right)$ and $b_{0}\left(y_{0} \mid \sigma\right)$ for the agent and the investor respectively is optimal at state $y_{0}$ if there is no other incentive-compatible contract $\tilde{\sigma}$ with the same payoff for the agent, but with a higher payoff for the investor: $a_{0}\left(y_{0} \mid \tilde{\sigma}\right)=a_{0}\left(y_{0} \mid \sigma\right)$ and $b_{0}\left(y_{0} \mid \tilde{\sigma}\right)>b_{0}\left(y_{0} \mid \sigma\right)$. This definition is based on the time zero payoffs.

In a similar manner, letting $a_{t}\left(y_{t} \mid \sigma_{t}\right)$ and $b_{t}\left(y_{t} \mid \sigma_{t}\right)$ denote continuation payoffs for the agent and the investor under a continuation contract $\sigma_{t}$, we can define an optimal continuation contract at any point of time:

Definition 1 An incentive-compatible continuation contract $\sigma_{t}$ is optimal at state $y_{t}$ if there is no other incentive-compatible continuation contract $\tilde{\sigma}_{t}$ such that $a_{t}\left(y_{t} \mid \tilde{\sigma}_{t}\right)=a_{t}\left(y_{t} \mid \sigma_{t}\right)$ and $b_{t}\left(y_{t} \mid \tilde{\sigma}_{t}\right)>b_{t}\left(y_{t} \mid \sigma_{t}\right)$.

We may think about an optimal continuation contract as the best incentive-compatible contract for the investor that can be written at time $t$, given the agent receives a certain continuation payoff. Note that the definition of the optimality of a continuation contract at time $t$ does not depend on what happened before time $t$. A natural question is whether a contract that is optimal at time zero will remain optimal in the future. To address this issue we need the following definition:

Definition 2 An incentive-compatible contract is sequentially optimal if at any point of time, after any history, its continuation contracts are optimal.

The definition of an optimal contract does not ensure its optimality in the future, while the definition of a sequentially optimal contract does not guarantee that such a contract exists. However, it is natural to conjecture that an optimal contract is also sequentially optimal:

Conjecture 1 There exists a sequentially optimal contract.

In other words, we conjecture that the optimal contract not only maximizes the investor's expected payoff at time zero for a given initial payoff for the agent, but also maximizes the investor's continuation payoff at any point of time in the future, given the corresponding continuation payoff for the agent. Although the statement of Conjecture 1 is true in the case 
when the cash flows are independent over time ${ }^{5}$, there is no obvious way to prove Conjecture 1 when the cash flows are correlated over time. We will show that a sequentially optimal contract exists. We will do it in two steps. First, we conjecture that the optimal contract is sequentially optimal. We will use this conjecture to justify our recursive algorithm to derive an optimal contract $\sigma^{*}$. Second, we will verify that the derived contact $\sigma^{*}$ is optimal by showing there is no other contract that can improve on $\sigma^{*}$.

\subsection{Sequentially Optimal Contract}

In this section, we derive a sequentially optimal contract using a recursive procedure. This procedure requires, that at any point of time, all the relevant information contained in the history is summarized by the two state variables: the realization of the last cash flow and the agent's continuation payoff. Conjecture 1 allows us to split the multidimensional optimization problem (6)-(8) into a sequence of optimization problems of smaller dimensionality.

An optimal contract solves the investor's problem given by (6)-(8). The solution of the problem gives us the function $b_{0}\left(y_{0}, a_{0}\right)$ that represents the highest possible payoff attainable by the investor, given the payoff $a_{0}$ for the agent and the initial state $y_{0}$. We will refer to $b_{0}\left(y_{0}, a_{0}\right)$ as the continuation function at time zero. In a similar manner, we can define continuation functions at any point of time in the future.

In each period $t<T$, the sequence of the following events takes place. First, the agent privately observes the realization of the cash flow $Y_{t}$. If $Y_{t}=y_{H}$, the agent decides whether to pay the cash flow to the investor, or divert it for his own consumption and report $y_{L}$. If $Y_{t}=y_{L}$, the agent has no choice other than to report the cash flow $y_{L}$. Given the agent's payment, the investor, in accordance with the contract, makes a decision regarding the termination of the project. In the case of termination, the agent's payoff is zero, and the investor's payoff is $L_{t}$. If the project is not terminated, it continues to operate in the next period. For each period, we consider the start-of-period, intra-period (just prior to the termination decision), and end-of-period continuation functions, denoted by $b_{t}^{y}\left(y_{t-1}, a_{t}^{y}\right)$, $b_{t}^{d}\left(y_{t}, a_{t}^{d}\right), b_{t}^{e}\left(y_{t}, a_{t}^{e}\right)$ correspondingly, where $a_{t}^{y}, a_{t}^{d}$, and $a_{t}^{e}$ denote the agent's continuation payoffs at the start, middle and end of period $t$ respectively, as shown in Figure 3.

In particular, $b_{t}^{y}\left(y_{t-1}, a_{t}^{y}\right)$ denotes the highest possible payoff attainable by the investor in period $t$ before the cash flow $Y_{t}$ is realized, given the agent's continuation payoffs $a_{t}^{y}$ at the beginning of period $t$ and the realization $y_{t-1}$ of the cash flow $Y_{t-1} ; b_{t}^{d}\left(y_{t}, a_{t}^{d}\right)$ denotes the highest possible payoff attainable by the investor in period $t$ after the cash flow $Y_{t}$ is realized but before the liquidation decision is made, given the agent's continuation payoffs $a_{t}^{d}$ at that time and the realization $y_{t}$ of the cash flow $Y_{t} ; b_{t}^{e}\left(y_{t}, a_{t}^{e}\right)$ denotes the highest possible payoff attainable by the investor in period $t$ after the liquidation decision is made, given the agent's continuation payoffs $a_{t}^{e}$ at the end of period $t$ and the realization $y_{t}$ of the cash flow $Y_{t}$.

\footnotetext{
${ }^{5}$ See for example, DeMarzo and Fishman (2003)
} 


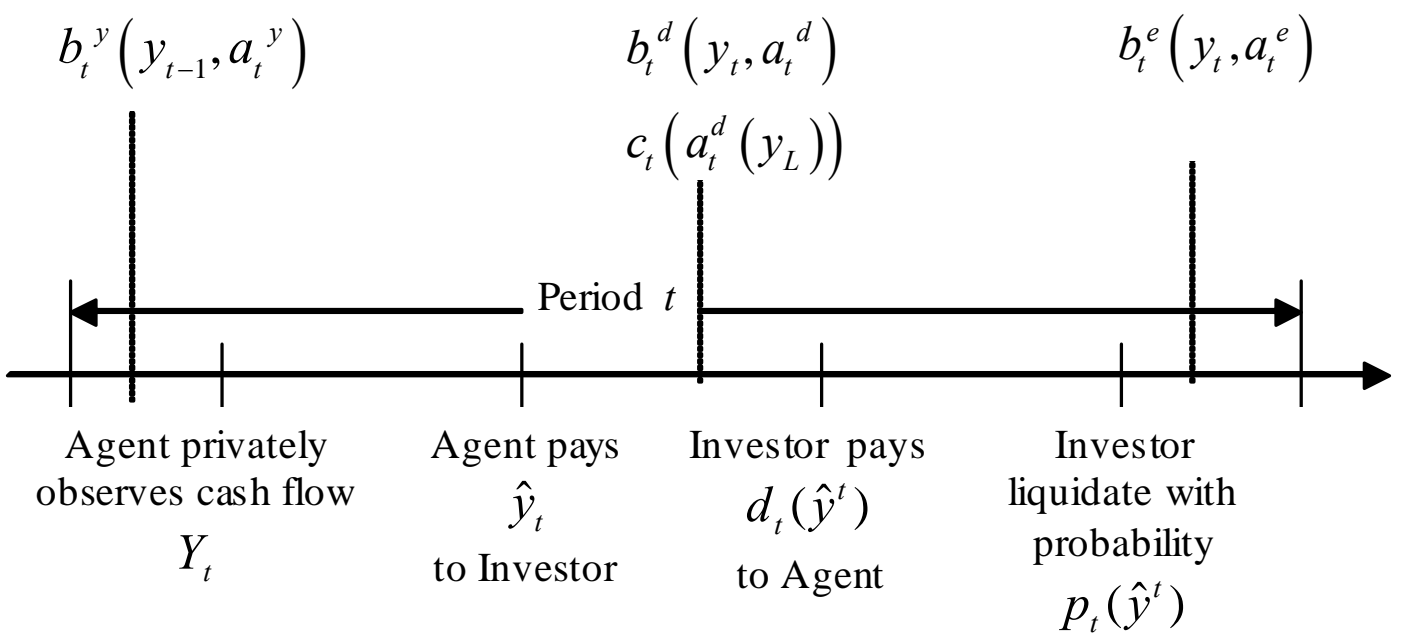

Figure 3: Continuation functions

Conjecture 1 allows us to calculate the optimal contract recursively. If a continuation contract $\sigma_{t+1}$ is optimal at time $t$, then we can use $\sigma_{t+1}$ to calculate a continuation contract $\sigma_{t}$ that is optimal at time $t-1$. In particular, given continuation function $b_{t+1}^{y}$, we can obtain continuation function $b_{t}^{e}$, taking into account discounting between periods. In order to calculate continuation function $b_{t}^{d}$, we calculate the optimal liquidation policy and the optimal transfer in period $t$. Along the way, we calculate the agent's deviation payoff $c_{t}\left(a_{t}^{d}\left(y_{L}\right)\right)$ as a function of the agent' equilibrium payoff $a_{t}^{d}\left(y_{L}\right)$ in the low state. Function $b_{t}^{y}$ is obtained from $b_{t}^{d}$ by optimizing the investor's payoff at the beginning of period $t$, subject to the incentive compatibility constraint and the promise keeping constraint.

We start computing the continuation functions from the final period $T$. At the end of the final period, the project is liquidated, and no cash flows will be generated in the future. However, a transfer from the investor to the agent is allowed. Due to the limited liability of the agent, this transfer must be non-negative. Hence, the continuation function at the end of the last period is given by

$$
b_{T}^{e}\left(y_{T}, a_{T}^{e}\right)=\left\{\begin{array}{l}
-a_{T}^{e} \text { for } a_{T}^{e} \geq 0 \\
-\infty \text { for } a_{T}^{e}<0
\end{array},\right.
$$

where the agent's continuation payoff $a_{T}^{e}$ is the final payment from the investor. 


\subsection{Parametric Representation of Continuation Payoffs}

In this subsection, we introduce a convenient way to represent the agent's continuation payoffs. Since the cash flows are correlated, the agent's continuation payoffs depend not only on the terms of the contact, but also on the last cash flow realization, which determines the distribution of the future cash flows. It turns out, as we will see later, this dependence can be conveniently incorporated into the agent's continuation payoffs, if these payoffs are represented in terms of the values of the cash flows that the agent can steal during a certain time interval.

For continuous time $\tau \in \Re$, let $n_{\tau}$ be the biggest integer, such that $n_{\tau} \leq \tau$, and $l_{\tau}=\tau-n_{\tau}$. We will use $\tau$ to denote a time interval of $n_{\tau}$ periods and the fraction $l_{\tau}$ of the next period. Given $Y_{t}=y$, the value of the cash flows that can be diverted by the agent during time $\tau$ starting from period $t+1$ is given by

$$
V_{\tau}(y) \equiv E\left[\sum_{k=1}^{n_{\tau}} \beta^{k}\left(Y_{t+k}-y_{L}\right)+l_{\tau} \beta^{\left(n_{\tau}+1\right)}\left(Y_{t+n_{\tau}+1}-y_{L}\right) \mid Y_{t}=y\right]
$$

Here, we assume that if the project operates a fraction $l_{\tau}$ of a period, then only the fraction $l_{\tau}$

of the cash flow realized in that period is counted. Alternatively, we can say that $l_{\tau}$ represents the probability that the agent will be allowed to run the project in the period $t+n_{\tau}+1$. Note that due to the Markov property of the cash flows, the current date $t$ is irrelevant for the value of $V_{\tau}$.

Function $V_{\tau}$ has a number of good properties.

Lemma 2 Function $V_{\tau}\left(Y_{t}\right)$ is continuous, strictly increasing in $\tau$, and is piecewise linear, with the right-hand-side derivative

$$
\frac{\partial V_{\tau}(y)}{\partial \tau}=\beta^{\left(n_{\tau}+1\right)} E\left[\left(Y_{t+n_{\tau}+1}-y_{L}\right) \mid Y_{t}=y\right] .
$$

Moreover,

$$
V_{\tau+1}\left(Y_{t-1}\right)=E\left[\beta\left(\left(Y_{t}-y_{L}\right)+V_{\tau}\left(Y_{t}\right)\right) \mid Y_{t-1}\right]
$$

Proof. See Appendix.

The equation (14) is self-evident. Its left-hand side is the value of the cash flows that can be stolen during time $\tau+1$, while the right-hand side represents the same value as a sum of the next period excess cash flow $\left(Y_{t}-y_{L}\right)$, and the value of the cash flows that can be stolen during time $\tau$. The derivative (13) is obtained from (12), using the fact that $l_{\tau}=\tau-n_{\tau}$.

We will use $V_{\tau}(\cdot)$ to represent continuation payoffs for the agent. For example, if $Y_{t}=y_{L}$, and the agent's continuation payoff at the end of period $t$ is $a>0$, then there exists unique $\tau>0$ such that $a=V_{\tau}\left(y_{L}\right)$. Thus, given state $y_{L}$, the agent's continuation payoff of $a$ is 
equivalent to the expected value of the cash flows that can be stolen between time $t+1$ and time $t+1+\tau$.

Two values of the agent's continuation payoffs have special meaning: $V_{1}\left(y_{t}\right)$ is the expected present value of the cash flow that can be stolen in the next period by the agent, while $V_{T-t}\left(y_{t}\right)$ is the expected present value of the cash flows that the project can generate if it operates until the final date $T$ without liquidation. Agent's continuation payoffs in period $t$ that are less than $\lambda V_{1}\left(y_{t}\right)$ cannot be implemented without liquidating the project with positive probability at the end of period $t$.

The only case when a continuation contract does not ever require liquidation is when the agent's continuation payoff is no less than $\lambda V_{T-t}\left(y_{t}\right)$, which is the value of the cash flows the agent is able to steal running the project until the final date $T$. The optimal contract looks especially easy in this situation. The investor pays to the agent the difference between the agent's continuation payoff and $\lambda V_{T-t}\left(y_{t}\right)$ immediately and lets the agent consume the fraction $\lambda$ of all subsequent excess cash flows. In this case, the continuation function at the end of period $t$ is given by

$$
b_{t}^{e}\left(y_{t}, a_{t}^{e}\right)=\sum_{s=t+1}^{T} \beta^{s-t} E\left[Y_{s} \mid Y_{t}=y_{t}\right]-a_{t}^{e} \text { for } a_{t}^{e} \geq \lambda V_{T-t}\left(y_{t}\right) .
$$

When the agent continuation payoff $a_{t}^{e}$ is below $\lambda V_{T-t}\left(y_{t}\right)$, the threat of liquidation must be real. Otherwise, the agent can steal all the subsequent excess cash flows and get the payoff $\lambda V_{T-t}\left(y_{t}\right)$.

\subsection{Derivation of the Sequentially Optimal Contract}

Now, we are in a position to present the algorithm which will allow us to derive the sequentially optimal contract $\sigma^{*}$. This algorithm consists of three steps for each period.

\subsubsection{Step One: Liquidation Problem}

Consider the problem the investor faces after the cash flow announcement but before the liquidation decision. If the project is terminated in period $t$, the investor's payoff is $L_{t}$, while the agent gets nothing. Given the continuation function $b_{t}^{e}\left(y_{t}, \cdot\right)$ and the agent's continuation payoff $a_{t}^{d}$, the optimal probability of liquidation $p_{t}$ solves

$$
\begin{aligned}
b_{t}^{d}\left(y_{t}, a_{t}^{d}\right) & =\max _{p_{t}, a_{t}^{e}}\left(1-p_{t}\right) b_{t}^{e}\left(y_{t}, a_{t}^{e}\right)+p_{t} L_{t} \\
\text { s.t. } a_{t}^{d} & =\left(1-p_{t}\right) a_{t}^{e}, \\
a_{t}^{e} & \geq \lambda V_{1}\left(y_{t}\right), \\
p_{t} & \in[0,1]
\end{aligned}
$$


where equation (17) ensures that the agent's continuation payoff $a_{t}^{d}$ before the liquidation decision is consistent with the continuation payoff $a_{t}^{e}$ after the liquidation decision. Constraint (18) reflects the fact that once the project is allowed to continue into the next period, the agent's end-of-period payoff must be at least as high as the expected present value of the cash flow $a^{L}\left(y_{t}\right) \equiv \lambda V_{1}\left(y_{t}\right)$ that the agent is capable of stealing in the next period. This also implies that if the agent's intra-period continuation payoff $a_{t}^{d}$ is below $a_{t}^{L}\left(y_{t}\right)$, the investor must liquidate the project in period $t$ with positive probability.

The next proposition states that the project is terminated with positive probability if and only if $a_{t}^{d}\left(Y_{t}\right)$ drops below $a^{L}\left(Y_{t}\right)$.

Proposition 2 The probability of the termination in period $t$ is given by

$$
p\left(Y_{t}, a_{t}^{d}\right)=\left\{\begin{array}{c}
\frac{a^{L}\left(Y_{t}\right)-a_{t}^{d}}{a^{L}\left(Y_{t}\right)} \text { for } a_{t}^{d} \in\left[0, a^{L}\left(Y_{t}\right)\right) \\
0 \text { for } a_{t}^{d} \geq a^{L}\left(Y_{t}\right)
\end{array} .\right.
$$

The intra-period continuation function $b_{t}^{d}\left(Y_{t}, a_{t}^{d}\right)$ is obtained from the end-of-period continuation function $b_{t}^{e}\left(Y_{t}, \cdot\right)$ as follows:

$$
b_{t}^{d}\left(Y_{t}, a_{t}^{d}\right)=\left\{\begin{array}{ccc}
-\infty & \text { for } & a_{t}^{d}\left(Y_{t}\right)<0 \\
\frac{a_{t}^{d}}{a^{L}\left(Y_{t}\right)} b_{t}^{e}\left(Y_{t}, a^{L}\left(Y_{t}\right)\right)+\left(1-\frac{a_{t}^{d}}{a^{L}\left(Y_{t}\right)}\right) L_{t} & \text { for } \quad a_{t}^{d} \in\left[0, a^{L}\left(Y_{t}\right)\right) . \\
b_{t}^{e}\left(Y_{t}, a_{t}^{d}\right) & \text { for } & a_{t}^{d}>a^{L}\left(Y_{t}\right)
\end{array} .\right.
$$

If the project is not terminated in period the agent's continuation payoff evolves as follows:

$$
a_{t}^{e}=\min \left(\lambda V_{T-t}\left(Y_{t}\right), \max \left(a_{t}^{d}, a^{L}\left(Y_{t}\right)\right)\right)
$$

The proof of Proposition 2 is in the Appendix. It is strongly recommended to finish reading this section first before reading the proof, since the proof relies on other results of this section. We prove Proposition 2 and the other propositions in this section all together through a backward induction argument. Assuming that the statements of these propositions are true in the subsequent periods, we prove that they must be true in the current period.

The intuition behind this result is simple. The termination of the project is inefficient. Therefore, the investor finds it optimal to refrain from early liquidation, unless she has exhausted all other means of providing proper incentives for the agent. Once the investor is in a position in which she has to resort to the liquidation, the probability of the liquidation is the smallest one that allows her to implement the agent's continuation payoff. This probability is proportional to how much the agent's continuation payoff $a_{t}^{d}$ is below the minimal implementable payoff $a^{L}\left(Y_{t}\right)$. If $a_{t}^{d}<a^{L}\left(Y_{t}\right)$, and the project was not terminated in period $t$, the agent's continuation payoff at the end of period $t$ is increased to $a^{L}\left(Y_{t}\right)$. 


\subsubsection{Step Two: Intra-Period Agency Problem}

At every point of time, the optimal contract governing the relationship between the investor and the agent in the future is characterized by two state variables: the realization of the last cash flow that determines the distribution of the future cash flows, and the promised payoff for the agent. After observing the realization of $Y_{t}$, the agent must report the cash flow to the investor. Let $a_{t}^{d}\left(y_{t}\right)$ denote the continuation payoff for the agent associated with the optimal contract if the agent truthfully announces the realization of $y_{t}$.

An important question is what the agent's continuation payoff would be if he reports $y_{L}$ instead of $y_{H}$. The contract specifies future payments to the agent and termination probabilities as a function of the history of the agent's reports. In this sense, the terms of the future contract do not depend on whether or not the agent was honest. However, $Y_{t}$ determines the distribution of future cash flows and, therefore, is relevant for the agent's continuation payoff under the continuation contract. If the agent cheats, his continuation payoff, as we will see, would be higher than $a_{t}^{d}\left(y_{L}\right)$, since he faces better prospects regarding future cash flows.

For the contract $\sigma^{*}$, let $c_{t}\left(a_{t}^{d}\left(y_{L}\right)\right)$ denote the continuation payoff for the agent if he chooses to report $y_{L}$ when $y_{H}$ was actually realized in period $t$. We write it as a function of

$a_{t}^{d}\left(y_{L}\right)$, since $a_{t}^{d}\left(y_{L}\right)$ fully determines the future terms of the optimal contract after $y_{L}$ was reported. We will refer to $c_{t}$ as the agent's deviation continuation payoff function.

Given the intra-period continuation function $b_{t}^{d}$, the start-of-period continuation function $b_{t}^{y}$ is the solution of the following problem:

$$
\begin{aligned}
b_{t}^{y}\left(Y_{t-1}, a_{t}^{y}\right) & =\max _{a_{t}^{d}(\cdot)} E_{t}\left[Y_{t}+b_{t}^{d}\left(Y_{t}, a_{t}^{d}\left(Y_{t}\right)\right) \mid Y_{t-1}\right] \\
\text { s.t. }(\mathrm{IC}) a_{t}^{d}\left(y_{H}\right) & \geq c_{t}\left(a_{t}^{d}\left(y_{L}\right)\right)+\lambda\left(y_{H}-y_{L}\right) \\
(\mathrm{PK}) \quad a_{t}^{y} & =E_{t}\left[a_{t}^{d}\left(Y_{t}\right) \mid Y_{t-1}\right] \\
(\mathrm{IR}) \quad a_{t}^{d}(\cdot) & \geq 0 .
\end{aligned}
$$

The start-of-period continuation payoff for the investor is the conditional expectation of the sum of the cash flow $Y_{t}$ and the intra-period continuation payoff, given by the function $b_{t}^{d}$. The intra-period continuation payoff for the agent conditional on realization of $Y_{t}, a_{t}^{d}\left(y_{L}\right)$ and $a_{t}^{d}\left(y_{H}\right)$ must satisfy the incentive compatibility constraint (IC), and the promise keeping constraint $(\mathrm{PK})$, and the individual rationality constraint (IR). The first constraint insures that the agent has no incentive to divert the high cash flow. The second one says that the payoff $a_{t}^{y}$ promised to the agent at the start of period $t$ is equal to the expected intra-period continuation payoff. Since the agent has limited liability, his continuation payoff cannot be negative. 
In the formulation of the problem (22), we explicitly use the conjecture that the contract $\sigma^{*}$ results in the continuation payoffs lying on the upper frontiers of the payoff possibility sets, represented by the continuation functions $b_{t}^{d}\left(Y_{t}, \cdot\right)$.

Proposition 3 The incentive compatibility constraint binds: for $t=1, \ldots, T$

$$
a_{t}^{d}\left(y_{H}\right)=c_{t}\left(a_{t}^{d}\left(y_{L}\right)\right)+\lambda\left(y_{H}-y_{L}\right) .
$$

Proof. See Appendix.

The proof is interconnected with the proofs of the other propositions in this section.

Proposition 3 allows us to calculate function $c_{t}$. We will represent the agent's continuation payoffs using functions $V_{\tau}(\cdot)$. For any $a_{t}^{d} \leq \lambda V_{T-t}\left(y_{t}\right)$, there is a unique parameter $\tau \leq T-t$ such that $a_{t}^{d}=\lambda V_{\tau}\left(y_{t}\right)$.

Proposition 4 Given contract $\sigma^{*}$, if the agent's continuation payoff in the state $y_{L}$ is $\lambda V_{\tau}\left(y_{L}\right)$ for some $0 \leq \tau \leq T-t$, then the agent's continuation payoff in deviation is $\lambda V_{\tau}\left(y_{H}\right)$ :

$$
c_{t}\left(\lambda V_{\tau}\left(y_{L}\right)\right)=\lambda V_{\tau}\left(y_{H}\right) \text { for } 0 \leq \tau \leq T-t
$$

Proof. See Appendix.

The terms of a continuation contract depend only on the reported cash flows. In this respect, if the agent reports the low cash flow in the current period he faces the same continuation contract whether or not the true cash flow realization was low. However, the agent's continuation payoff depends on the true cash flow realization, since the distribution of future cash flows is a function of the current cash flow realization. Proposition 4 says that if the agent's continuation payoff in the low state is equal to the expected present value of the cash flows that the agent can steal during next $\tau$ periods, then the agent's continuation payoff in deviation is equal to the expected present value of the cash flows that the agent can steal during next $\tau$ periods, given the high state in the current period.

Let $\sigma_{t}^{*}\left(y_{L}\right)$ denote the continuation contract after the agent reports the low cash flow in period $t$. Given the probability of liquidation $p_{t}$ in period $t$ under $\sigma_{t}\left(y_{L}\right)$, we can represent the agent's continuation payoff in equilibrium as the expectation of the agent's continuation payoffs in the next period:

$$
a_{t}^{d}\left(y_{L}\right)=E\left[\beta\left(1-p_{t}\right) a_{t+1}^{d}\left(Y_{t+1}\right) \mid Y_{t}=y_{L}\right]
$$

where $a_{t+1}^{d}\left(Y_{t+1}\right)$ denotes the continuation payoff for the agent in period $t+1$ under the continuation contract $\sigma_{t}^{*}\left(y_{L}\right)$ conditional on the cash flow $Y_{t+1}$. Similarly, if the agent reports 


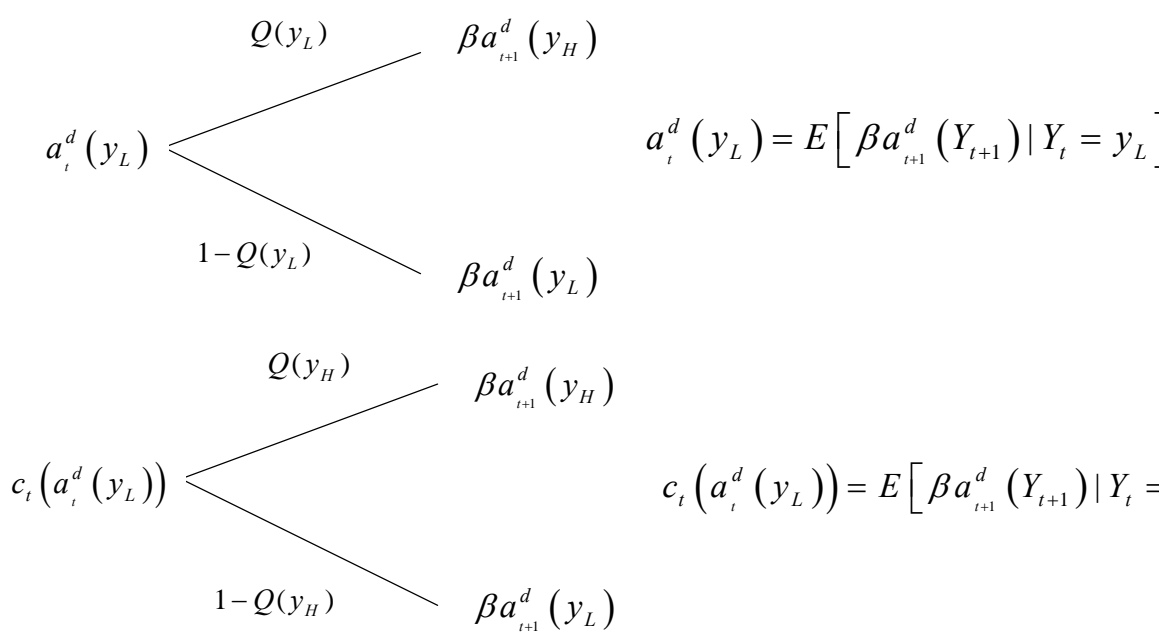

Figure 4: Computing function $c_{t}$ for the case without liquidation in period $t$.

the low cash flow in period $t$, when, in fact, the high cash flow realized, the same contract results in the following continuation payoff after the deviation:

$$
c_{t}\left(a_{t}^{d}\left(y_{L}\right)\right)=E\left[\beta\left(1-p_{t}\right) a_{t+1}^{d}\left(Y_{t+1}\right) \mid Y_{t}=y_{H}\right] .
$$

Given (28)-(29), one can calculate function $c_{t}$ using backward induction. Figure 4 gives graphical interpretation of the relationship between $a_{t}^{d}\left(y_{L}\right)$ and $c_{t}\left(a_{t}^{d}\left(y_{L}\right)\right)$ for the case with $p_{t}=0$.

Proposition 4 allows us to calculate the evolution of the agent's continuation payoffs. Since the continuation payoffs $a_{t}^{d}\left(y_{L}\right)$ and $a_{t}^{d}\left(y_{H}\right)$ satisfy the promise-keeping constraint $(\mathrm{PK})$ and the incentive compatibility constraint (IC), which is binding, we can solve equations (PK) and (IC) for $a_{t}^{d}\left(y_{L}\right)$ and $a_{t}^{d}\left(y_{H}\right)$ as functions of $a_{t}^{y}$. This yields the following:

Proposition 5 For the start-of-period continuation payoff given by $a_{t}^{y}=\frac{1}{\beta} \lambda V_{\tau+1}\left(Y_{t-1}\right)$, with $0 \leq \tau \leq T-t$, the agent's intra-period continuation payoff is given by

$$
a_{t}^{d}\left(Y_{t}\right)=\lambda V_{\tau}\left(Y_{t}\right)+\lambda\left(Y_{t}-y_{L}\right)
$$

The start-of-period continuation function is obtained as follows:

$$
b_{t}^{y}\left(Y_{t-1}, \frac{1}{\beta} \lambda V_{\tau+1}\left(Y_{t-1}\right)\right)=E_{t}\left[Y_{t}+b_{t}^{d}\left(Y_{t}, \lambda V_{\tau}\left(Y_{t}\right)+\lambda\left(Y_{t}-y_{L}\right)\right) \mid Y_{t-1}\right] .
$$




\subsubsection{Step Three: Discounting between Periods}

So far, we have demonstrated that knowing the end-of-period continuation function $b_{t}^{e}$, one can obtain the intra-period continuation function $b_{t}^{d}$, and then the start-of-period continuation function $b_{t}^{y}$. To complete our recursive characterization of the optimal contract, we derive the continuation function $b_{t-1}^{e}$ from $b_{t}^{y}$.

With the exception of the payoff discounting, nothing takes place between the end of the prior period and the beginning of the next period. Hence, if the contract results in the payoff of $a_{t-1}^{e}$ at the end of period $t-1$, then the agent's payoff at the start of period $t$ must be $a_{t-1}^{e} / \beta$. The above argument, combined with the fact that the investor discounts future cash flows using the same discount factor $\beta$, yields the following:

Proposition 6 Given the start-of-period continuation function $b_{t}^{y}$, the continuation function at the end of period $t$ is given by

$$
b_{t-1}^{e}\left(y_{t-1}, a_{t-1}^{e}\right)=\beta b_{t}^{y}\left(y_{t-1}, \frac{a_{t-1}^{e}}{\beta}\right) .
$$

\subsubsection{Summary of the Algorithm}

Propositions 2, 5, 6, and 4, yield the algorithm of computing recursively the functions $b_{t}^{e}, b_{t}^{d}, b_{t}^{y}$, and $c_{t}$ starting from the end of the last period $T$. In general, the functions $b_{t}^{e}, b_{t}^{d}, b_{t}^{y}$ are too complicated to be written explicitly. However, the evolution of the agent's continuation payoff can be described compactly, as is done below:

$$
\begin{aligned}
a_{t-1}^{e} & =\lambda V_{\tau}\left(Y_{t-1}\right) \\
& \rightarrow a_{t}^{y}=\lambda V_{\tau}\left(Y_{t-1}\right) / \beta \\
& \rightarrow a_{t}^{d}\left(Y_{t}\right)=\lambda V_{\tau-1}\left(Y_{t}\right)+\lambda\left(Y_{t}-y_{L}\right) \\
& \rightarrow a_{t}^{e}=\min \left(\lambda V_{T-t}\left(Y_{t}\right), \max \left(a_{t}^{d}\left(Y_{t}\right), a^{L}\left(Y_{t}\right)\right)\right)
\end{aligned}
$$

During the transition from the end of period $t-1$ to the beginning of period $t$, the agent's continuation payoff is adjusted by the discount factor $\beta$. The transition from $a_{t}^{y}$ to $a_{t}^{d}\left(Y_{t}\right)$ provides the agent with an incentive to report the realization of the cash flow $Y_{t}$ truthfully. Comparing to $a_{t}^{y}$, the agent's continuation payoff is going to increase if $Y_{t}=y_{H}$, and decrease if $Y_{t}=y_{L}$ :

$$
a_{t}^{d}\left(y_{L}\right)<a_{t}^{y}<a_{t}^{d}\left(y_{H}\right)
$$

Transition from $a_{t}^{d}$ to $a_{t}^{e}$ is characterized by the liquidation probability $p_{t}$ and the transfer $d_{t}$.

The evolution of agent's continuation payoffs can be characterized by three regions, as shown in Figure 5. The project can be liquidated only if the agent's continuation payoff 


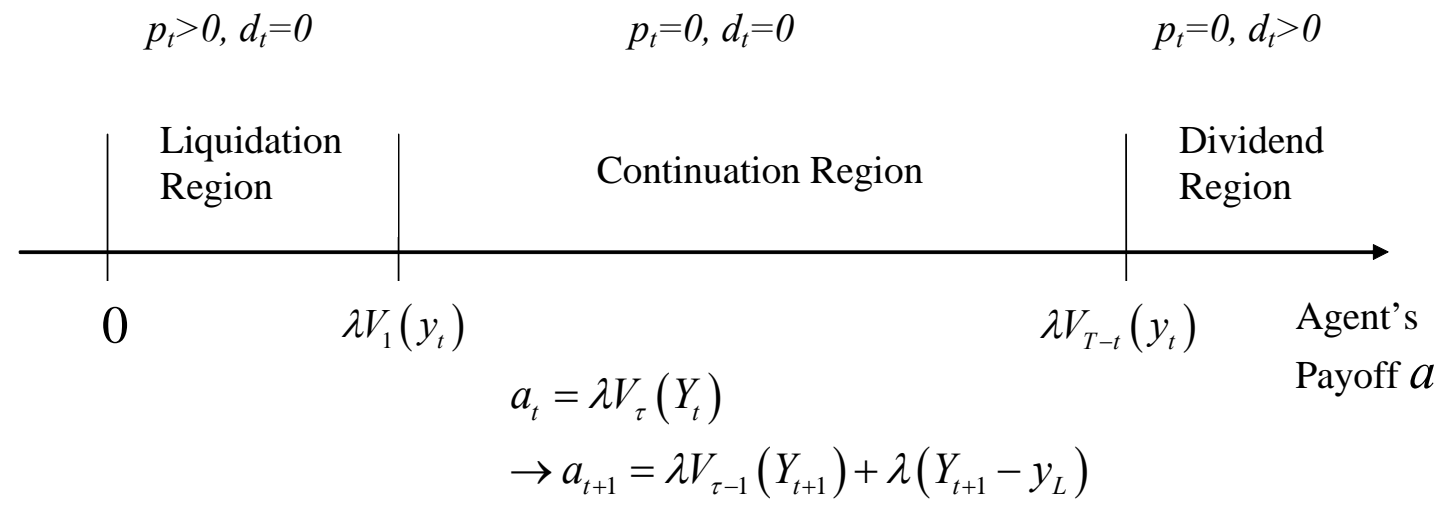

Figure 5: Three regions of the agent's continuation payoffs

$a_{t}^{d}$ drops into the liquidation region whose upper boundary is given by $a^{L}\left(y_{t}\right)=\lambda V_{1}\left(y_{t}\right)$. In the liquidation region, the transfer from the investor to the agent is always zero and the probability of liquidation is proportional to how much the agent's continuation payoff is below the liquidation boundary.

$$
p_{t}\left(a_{t}^{d}\left(y_{t}\right)\right)=\max \left(\frac{a^{L}\left(y_{t}\right)-a_{t}^{d}\left(y_{t}\right)}{a^{L}\left(y_{t}\right)}, 0\right) .
$$

In the continuation region, there is no liquidation, but the transfer is also equal to zero. The agent's continuation payoff is affected by the realization of $Y_{t}$ in two ways. First, the current cash flow $Y_{t}$ directly affects the continuation payoff through the term $\lambda\left(Y_{t}-y_{L}\right)$. This term represents the value of the realized cash flow without taking into account an impact of this cash flow realization on the subsequent cash flows. Second, the cash flow $Y_{t}$ affects the agent's continuation payoff through its impact on the distribution of the future cash flows, which is reflected in the term $\lambda V_{\tau-1}\left(Y_{t}\right)$.

The dividend region is where the first best is implemented. When the agent's continuation payoff $a_{t}^{d}\left(y_{t}\right)$ is above $\lambda V_{T-t}\left(y_{t}\right)$, the investor pays to the agent $d_{t}=a_{t}^{d}\left(y_{t}\right)-\lambda V_{T-t}\left(y_{t}\right)$ in period $t$. Once the agent's continuation payoff reaches the dividend region, it always stays there. The the transfers to the agent in the subsequent periods are given by $d_{s}=\lambda\left(y_{s}-y_{L}\right)$ for $s>t$.

As an immediate consequence of the evolution of the agent's continuation payoff, we have the following lemma:

Lemma 3 The project is never liquidated in a period in which the high cash flow is reported.

Indeed, at the beginning of a period $t$, the agent's continuation payoff must be at least 
$a^{L}\left(y_{t-1}\right) / \beta=V_{1}\left(y_{t-1}\right) / \beta$, which is the expected value of the cash flow the agent is able to steal in this period. After the cash flow $y_{H}$ is reported, his continuation payoff can only increase. Thus, after a realization of the high cash flow, the agent's continuation payoff never goes down into the liquidation zone $\left[0, \lambda V_{1}\left(y_{t}\right)\right]$.

The parameter $\tau$ can be interpreted as the expected earliest default time. We will refer to $\tau$ simply as the earliest default time. Given the agent's continuation payoff $a_{t-1}^{d}=\lambda V_{\tau_{t-1}}\left(Y_{t-1}\right)$ in period $t-1$, the liquidation will not occur during the next $n_{\tau_{t-1}}$ periods, no matter what cash flows are reported in these periods. However, if the agent reports the low cash flow $n_{\tau_{t-1}}+1$ times in a row, the liquidation will occur in period $t+n_{\tau_{t-1}}$ with probability $l_{\tau_{t-1}}$. To see this, consider the evolution of the agent's continuation payoffs given by (31)-(34). If the agent reports the low cash flow in period $t$, his continuation payoff after the report will be $\lambda V_{\tau_{t-1}-1}\left(y_{L}\right)$ if the agent tells the truth and $\lambda V_{\tau_{t-1}-1}\left(y_{H}\right)$ if he lies. Thus, the agent's continuation payoff will be $\lambda V_{\tau_{t}}\left(Y_{t}\right)$ in period $t$, where $\tau_{t}=\tau_{t-1}-1$. The liquidation will not occur in period $t$ as long as $\tau_{t} \geq 1$. Now, one can see that if the agent reports the low cash flow in period $t+1$, his continuation payoff will be $\lambda V_{\tau_{t+1}}\left(Y_{t}\right)$, where $\tau_{t+1}=\tau_{t}-1$. Thus, the liquidation cannot occur before period $t+n_{\tau_{t-1}}$.

\subsubsection{Outline of the proof}

We are now in a position to prove that if there exists a sequentially optimal contract, then it must be the contract $\sigma^{*}$ that has been derived using the above algorithm. In this subsection, we only provide an intuitive explanations for the form of the optimal contract, while the Appendix contains formal proofs of all the statements made in this section.

Our proof is by induction that starts from the last period $T$. Assuming that all the properties of the contract $\sigma^{*}$ outlined above hold in periods $t+1$ through $T$, we prove that they must hold in period $t$ to maximize the investor's expected payoff in this period. In each period, assuming we know the function $b_{t}^{e}$, we solve the liquidation problem (16)-(19), whose

solution gives us the optimal liquidation probability $p_{t}$, and the continuation function $b_{t}^{d}$. The next step is to solve the intra-period agency problem (22)-(24) and obtain the continuation function $b_{t}^{y}$. The last step is the transition from the beginning of period $t$ to the end of the period $t-1$, which involves discounting between the periods.

To prove Proposition 2, we need to solve the liquidation problem (16)-(19), in which we maximize the investor's continuation payoff $b_{t}^{d}\left(y_{t}, a_{t}^{d}\right)$ before the liquidation decision, given continuation function $b_{t}^{e}$. A probabilistic liquidation means that we randomize between the payoff pair $\left(0, L_{t}\right)$ in the event of liquidation, and the pair $\left(a_{t}^{e}, b_{t}^{e}\left(y_{t}, a_{t}^{e}\right)\right)$ in the event of continuation, where the agent's payoff in the event of continuation is given by $a_{t}^{e}=a_{t}^{d} /\left(1-p_{t}\right)$, and $p_{t}$ is the probability of liquidation. Let $\left(\tilde{a}_{t}^{L}, b_{t}^{e}\left(y_{t}, \tilde{a}_{t}^{e}\right)\right)$ denote the payoff point of tangency of a line originating from the point $\left(0, L_{t}\right)$ to the upper frontier of the payoff possibility set at the end of the period $t$, given by the function $b_{t}^{e}$. Since the function $b_{t}^{e}$ is concave, the 


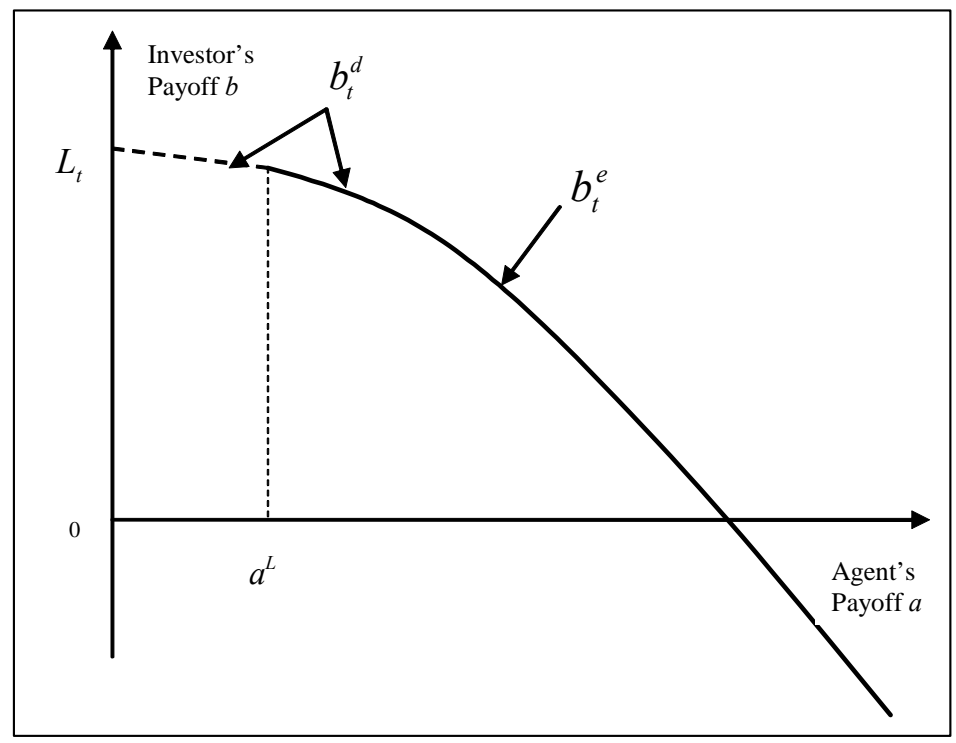

Figure 6: Continuation functions $b_{t}^{d}$ and $b_{t}^{e}$

continuation function $b_{t}^{d}$ is given by the line connecting the points $\left(0, L_{t}\right)$ and $\left(\tilde{a}_{t}^{L}, b_{t}^{e}\left(y_{t}, \tilde{a}_{t}^{e}\right)\right)$ on the interval $\left[0, \tilde{a}_{t}^{L}\right]$ and coincides with $b_{t}^{e}$ on $\left(\tilde{a}_{t}^{L}, \infty\right)$. We prove in the Appendix that $\tilde{a}_{t}^{L}=a_{t}^{L}\left(y_{t}\right)$. Figure 6 shows the function $b_{t}^{e}$ (solid line), which is defined on $\left[a^{L}, \infty\right]$, and the function $b_{t}^{d}$, which is obtained from $b_{t}^{e}$ by extending it over the liquidation region $\left[0, a^{L}\right]$ (dashed line).

Intuitively, we can think about the optimal contracting problem as minimizing the liquidation probabilities while keeping the contract incentive compatible. Due to the inefficiency of liquidation, the investor prefers to threaten the agent with the liquidation in the future rather than resorting to liquidation immediately. Only when no threat in the future can induce truth-telling in the current period does the investor liquidate the project.

We now discuss the intra-period agency problem (22)-(25). In each period $t$, the agent's incentives to tell the truth are given through the continuation payoffs $a_{t}^{d}\left(y_{t}\right)$. The agent's continuation payoff increases $\left(a_{t}^{d}\left(y_{H}\right)>a_{t}^{y}\right)$ when the high cash flow is reported, and decreases $\left(a_{t}^{d}\left(y_{L}\right)<a_{t}^{y}\right)$ when the low cash flow is reported. The incentive compatibility constraint (23) is satisfied only when the difference between $a_{t}^{d}\left(y_{H}\right)$ and $a_{t}^{d}\left(y_{L}\right)$ is wide enough. According to Proposition 3, in order to maximize the investor's payoff the incentive compatibility constraints (23) must bind, meaning that the difference between $a_{t}^{d}\left(y_{H}\right)$ and $a_{t}^{d}\left(y_{L}\right)$ is minimized.

To see why the (IC) constraint should bind, consider how the liquidation probability would be different when the (IC) constraint does not bind. The liquidation is likely to occur sooner after the low cash flow realization than after the high cash flow realization, since the payoff $a_{t}^{d}\left(y_{L}\right)$ is closer than the payoff $a_{t}^{d}\left(y_{H}\right)$ to the liquidation region $\left(0, a^{L}\right)$. Since the 
promise-keeping constraint (24) insures that the mean of $a_{t}^{d}\left(y_{H}\right)$ and $a_{t}^{d}\left(y_{L}\right)$ is fixed and is equal to $a_{t}^{y}$, relaxing the (IC) constraint leads to a lower payoff $a_{t}^{d}\left(y_{L}\right)$ after the low cash flow realization and to a higher payoff $a_{t}^{d}\left(y_{H}\right)$ after the high cash flow realization, comparing to the case when the (IC) constraint binds. Thus, relaxing the (IC) constraint leads to even higher chances of liquidation when liquidation is more likely, and even lower chances of liquidation when it is less likely. As a result, the expected loss associated with the liquidation increases. Thus, the (IC) constraint should bind.

\subsection{The Optimal Contract without Restrictions}

We assumed that Conjecture 1 is true when deriving the contract $\sigma^{*}$. The algorithm employed to derive the contract $\sigma^{*}$ ensures that if there exists a sequentially optimal contract, it must be the contract $\sigma^{*}$. However, it may be possible that the optimal contract, i.e. a contract that maximizes the investor's payoff at time zero, given the agent's payoff, is not sequentially optimal, i.e. it results in suboptimal payoffs after some histories. In this case, the contract $\sigma^{*}$ will remain incentive compatible, but will not maximize the investor's initial payoff.

In this section, we verify that the contract $\sigma^{*}$ is the optimal contract. We relied on Conjecture 1 in the formulation of the intra-period agency problem (22)-(24). According to this problem, the investor maximizes his continuation payoff at the start of period $t$, by choosing the agent's continuation payoffs $a_{t}^{d}\left(y_{L}\right)$ and $a_{t}^{d}\left(y_{H}\right)$, such that they satisfy the promise-keeping constraint $(\mathrm{PK})$ and the incentive compatibility constraint (IC). The (IC) constraint is written using the deviation payoff function $c_{t}$, which is associated with the continuation contract $\sigma_{t}^{*}$ after $y_{L}$ was reported in period $t$. We prove that the (IC) constraints bind for all $t \leq T$, and compute function $c_{t}$ recursively.

According to the properties of $\sigma^{*}$, if the agent's continuation payoff at the start of period $t$ is given by $a_{t}^{y}=\lambda V_{\tau}\left(Y_{t-1}\right) / \beta$, then his continuation payoff will be $a_{t}^{d}\left(Y_{t}\right)=\lambda V_{\tau-1}\left(Y_{t}\right)+$ $\lambda\left(Y_{t}-y_{L}\right)$, if he truthfully reports $Y_{t}$. Hence, the investor's continuation payoff will be $b_{t}^{d}\left(Y_{t}, a_{t}^{d}\left(Y_{t}\right)\right)$, if $Y_{t}$ is realized. We, however, have not considered continuation contracts that result in payoffs below the upper frontiers of the payoff possibility sets, represented by $b_{t}^{d}\left(Y_{t}, \cdot\right)$. It is not obvious that such contracts are inferior to $\sigma^{*}$, since their incentive compatibility constraints can be different from those associated with the contract $\sigma^{*}$.

The next proposition states the main result of this section.

Proposition $7 \sigma^{*}$ is the optimal contract.

Proof. See Appendix.

Below, we discuss the main elements of the proof. The contract $\sigma^{*}$ has two important properties. First, the incentive compatibility constraints always bind. Second, the project can be liquidated only if the agent's continuation payoff drops below $\lambda V_{1}\left(y_{L}\right)$, the lowest payoff value that can be implemented without liquidation. The first property says that the investor 
minimizes the distance between $a_{t}^{d}\left(y_{H}\right)$ and $a_{t}^{d}\left(y_{L}\right)$, subject to the (IC) constraint, while keeping the expectation of $a_{t}^{d}\left(Y_{t}\right)$ constant. Choosing continuation payoffs $\tilde{a}_{t}^{d}\left(y_{H}\right)>a_{t}^{d}\left(y_{H}\right)$ and $\tilde{a}_{t}^{d}\left(y_{L}\right)<a_{t}^{d}\left(y_{L}\right)$, so that the $(\mathrm{PK})$ holds, would result in lower continuation payoff for the investor. Choosing continuation payoffs $\tilde{a}_{t}^{d}\left(y_{H}\right)<a_{t}^{d}\left(y_{H}\right)$ and $\tilde{a}_{t}^{d}\left(y_{L}\right)>a_{t}^{d}\left(y_{L}\right)$, so that the $(\mathrm{PK})$ holds, would violate the (IC) constraint. According to Proposition 3, the incentive compatibility constraints bind for the contract $\sigma^{*}$, which means that if the function $b_{t}^{d}$ represents the upper frontier of the payoff possibility set (it does if $\sigma^{*}$ is the optimal contract), then the investor's payoff at the beginning of period $t$ is maximized when the difference between the agent's continuation payoffs $\tilde{a}_{t}^{d}\left(y_{L}\right)$ and $\tilde{a}_{t}^{d}\left(y_{H}\right)$ is minimized, subject to the incentive compatibility and the promise-keeping constraints.

To prove Proposition 7, we start by considering an arbitrary incentive compatible contract $\tilde{\sigma}$, whose incentive compatibility constraints do not necessarily bind. Let $\tilde{c}_{t}$ denote a deviation payoff function, associated with the contract $\tilde{\sigma}$. Since, by assumption, $\tilde{\sigma}$ is incentive compatible, the agent's continuation payoffs $\tilde{a}_{t}^{d}$, associated with $\tilde{\sigma}$, must satisfy

$$
\tilde{a}_{t}^{d}\left(y_{H}\right) \geq \tilde{c}_{t}\left(\tilde{a}_{t}^{d}\left(y_{L}\right)\right)+\lambda\left(y_{H}-y_{L}\right) \text { for all } t \leq T \text {. }
$$

One can show recursively that equation (36) implies that $\tilde{c}_{t}(a) \geq c_{t}(a)$ for all $a \geq 0$, and $t \leq T$.

The function $\tilde{c}_{t}$ determines the difference between $\tilde{a}_{t}^{d}\left(y_{H}\right)$ and $\tilde{a}_{t}^{d}\left(y_{L}\right)$. The greater the value of the function $\tilde{c}_{t}$, the greater this difference is. Since the continuation payoffs associated with the contract $\tilde{\sigma}$ lie below the upper frontier of the payoff possibility set, $\tilde{\sigma}$ can be an improvement on $\sigma^{*}$, only if $\tilde{\sigma}$ reduces the difference between the corresponding continuation payoffs for the agent. However, $\tilde{\sigma}$ never decreases this difference. Hence, $\tilde{\sigma}$ cannot be better than $\sigma^{*}$.

\subsection{Initiating the Contract}

The contract is initiated at time zero. The initial state $Y_{0}$ of the Markov cash flows and the continuation payoff $a_{0}^{e}\left(Y_{0}\right)$ for the agent uniquely determine the optimal contract between the agent and the investor. Thus, when $Y_{0}$ is commonly known, initiating the contract means choosing the payoffs $a_{0}^{e}\left(Y_{0}\right)$ and $b_{0}^{e}\left(y_{L}, a_{0}^{e}\left(y_{L}\right)\right)$ for the agent and for the investor, respectively.

The situation is not much different when the initial state $Y_{0}$ is unknown but its distribution is the common knowledge. Suppose, $Y_{0}$ is unknown, but both the agent and the investor assess that $\operatorname{Pr}\left(Y_{0}=y_{H}\right)=q_{H}$.

Proposition 8 If the optimal contract $\sigma^{*}$ implements payoffs $a_{0}^{e}\left(Y_{0}\right)$, and $b_{0}^{e}\left(Y_{0}, a_{0}^{e}\left(Y_{0}\right)\right)$, when the state $Y_{0}$ is known, then, when $Y_{0}$ is unknown, $\sigma^{*}$ is also optimal and implements payoffs $a_{0}=E\left[a_{0}^{e}\left(Y_{0}\right)\right]$, and $b_{0}\left(a_{0}\right)=E\left[b_{0}^{e}\left(Y_{0}, a_{0}^{e}\left(Y_{0}\right)\right)\right]$.

Proof. See Appendix. 
In the proof of Proposition 8, we use the fact that the contract $\sigma^{*}$ remains optimal in both states.

The contract sets initial continuation payoffs for the agent and the investor. Which pair of payoffs is chosen depends on the competitive environment. If the agent is a monopolist facing a competitive row of investors, he chooses the initial continuation payoff $a_{0}^{A}$, so that the investor is break-even:

$$
a_{0}^{A}=\sup \left\{a: b_{0}(a) \geq I-W\right\}
$$

Proposition 9 In the setting with a monopolistic agent and competitive investors, it is optimal for the agent to invest all his wealth in the project at date 0.

Proof. See Appendix.

On the other hand, if the investor has all the market power, she chooses to maximize her payoff:

$$
a_{0}^{I}=\arg \max _{a} b_{0}(a)
$$

In general, the initial payoff $a_{0}$ for the agent can be anything between $a_{0}^{I}$ and $a_{0}^{A}$ depending on the market power of the agent and the investor, with $a_{0}$ increasing with the agent's market power and decreasing with the investor's market power.

\section{The Implementation Result}

So far we have characterized the optimal contract in terms of the transfers between the agent and the investors, and the probabilities of liquidation of the project. In this section, we show that the optimal contract can be implemented using a credit line with performance pricing, equity and a coupon bond. We define these securities as follows:

Coupon Bond. A coupon bond represents the agent's commitment to make a coupon payments $x$ to the investor at the end of each period $t \leq T$. If the agent is unable to make the coupon payment, the firm is in default.

Credit Line with Performance Pricing. A credit line is characterized by a credit limit $C_{t}^{L}$ and an interest rate $r_{t}^{C}\left(M_{t-1}\right)$ charged on the balance $M_{t-1}$ on the credit line. Note that we allow the interest rate to be a function of the credit line balance. The interest payment on the outstanding balance is due at the end of each period. The credit limit $C_{t}^{L}$ determines the maximum amount of credit available for the agent in period $t$. Inability to make the current interest payment without exceeding the credit line limit leads to default. In addition, the credit line can have an initiation fee and restrictions on the dividend policy, which we will discuss later. 
Equity. The agent is allowed to use cash flows to pay dividends to the equity holders in proportion to their share of ownership.

Default occurs when the agent is unable to fulfil his financial obligations. Default leads to a probabilistic liquidation. Given an unmade payment $z_{t}$, the firm is liquidated with probability $p_{t}\left(z_{t}\right)$, while with probability $\left(1-p_{t}\left(z_{t}\right)\right)$, the investor forgives the unmade payment $z_{t}$, and lets the agent operate the firm in the next period ${ }^{6}$. In the even of liquidation, the investor sells the firm's assets and pockets the liquidation value $L_{t}$, while the agent gets nothing.

Theorem 2 The optimal contract can be implemented by a combination of equity, a coupon bond, and a credit line with an escalating interest rate. The agent holds fraction $\lambda$ of the equity, while the rest is held by the investor. The bond's coupon is equal to the verifiable cash flow:

$$
x=y_{L}
$$

The credit line has a credit limit given by

$$
C_{t}^{L}=V_{T-t}\left(y_{H}\right)-V_{1}\left(y_{H}\right) \text { for } t<T \text {, }
$$

and an interest rate $r_{t}^{C}$ that depends on the credit line balance as follows:

Let the balance $M_{t-1}$ on the credit line at the end of period $t-1$ be represented as a function of parameter $\tau$ :

$$
\left.M_{t-1}(\tau)=V_{T-t}\left(y_{H}\right)-V_{\tau}\left(y_{H}\right)\right)
$$

Then, the interest rate charged on this balance is given by

$$
r_{t}^{C}\left(M_{t-1}(\tau)\right)=\frac{V_{T-t}\left(y_{H}\right)-V_{\tau-1}\left(y_{H}\right)}{V_{T-t+1}\left(y_{H}\right)-V_{\tau}\left(y_{H}\right)}-1 .
$$

The agent can draw on the credit line to make interest payments. However, he is not allowed to borrow from the credit line to pay dividends.

In the event of default, the unmade payment $z_{t}$ results in the probability of liquidation

$$
p_{t}\left(z_{t}\right)=\frac{z_{t}}{V_{1}\left(y_{H}\right)}
$$

With probability $\left(1-p_{t}\left(z_{t}\right)\right)$, the project is not liquidated, and the unmade payment $z_{t}$ is forgiven.

Proof. : See Appendix.

\footnotetext{
${ }^{6}$ Although our definition of default is non-standard, it is consistent with the fact that creditors are often willing to write off a part of the debt instead of forcing bankruptcy. We can interpret the probabilistic liquidation as an uncertainty associated with the default procedure, which we do not model here.
} 


\begin{tabular}{c|c|c|l} 
& $\begin{array}{l}\text { Liquidation } \\
\text { Region }\end{array}$ & Continuation Region & $\begin{array}{l}\text { Dividend } \\
\text { Region }\end{array}$ \\
\hline 0 & $V_{1}\left(y_{H}\right)$ & $V_{T-t}\left(y_{H}\right) \begin{array}{l}\text { Agent's } \\
\text { Cont. } \\
\text { Payoff }\end{array}$
\end{tabular}

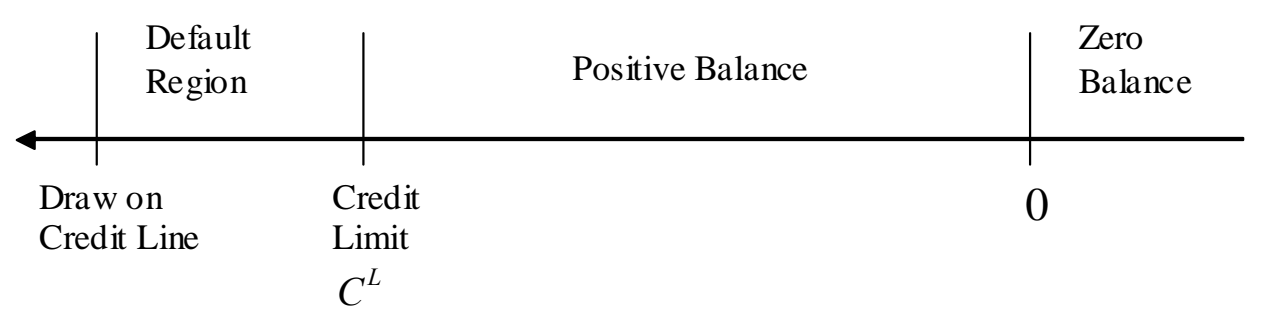

Figure 7: Agent's continuation payoff and credit line balance

In the optimal combination of securities, the roles of the coupon debt and equity are straightforward. The coupon debt is used to extract the verifiable cash flow $y_{L}$, while dividends paid to the equity holders represent a reward to the agent for repaying the credit line debt. Given his stake $\lambda$ in the firm's equity, the agent is indifferent between stealing cash flows and issuing the dividends. The role of the credit line with an escalating interest rate is more sophisticated. The balance on the credit line can be considered as a memory device that summarizes all the relevant information regarding the past cash flow realizations. The interest rate along with the credit limit determines the dynamics of the credit line balance and the timing of the default. The threat of losing control over the project induces the agent to pay the credit line.

To prove Theorem 2, we show that the evolution of the balance on the credit line reflects the evolution of the agent's continuation payoffs induced by the contract $\sigma^{*}$. Specifically, the parameters of the credit line are chosen so that the available credit is proportional to the agent's continuation payoff in the high state under the optimal contract $\sigma^{*}$ minus the liquidation boundary $\lambda V_{1}\left(y_{H}\right)$ :

$$
C_{t}^{L}-M_{t}=\left(a_{t}^{d}\left(y_{H}\right)-\lambda V_{1}\left(y_{H}\right)\right) / \lambda .
$$

As Figure 7 illustrates, zero balance on the credit line corresponds to the dividend threshold $\lambda V_{T-t}\left(y_{H}\right)$, while the balance equal to the credit limit corresponds to the liquidation threshold $\lambda V_{1}\left(y_{H}\right)$. An increase in the balance leads to a lower continuation payoff. Default occurs when the balance exceeds the credit limit. 
The agent uses all excess cash flows $\left(Y_{t}-y_{L}\right)$ to pay the credit line. Given the outstanding balance $M_{t-1}$ in period $t-1$ and the payment $\left(Y_{t}-y_{L}\right)$ by the agent in period $t$, the new balance becomes

$$
M_{t}=\left(1+r_{t}^{C}\left(M_{t-1}\right)\right) M_{t-1}-\left(Y_{t}-y_{L}\right) .
$$

When a cash flow is low, the agent has to draw on the credit line to make the interest payment, as long as his outstanding balance stays within the credit limit. On the other hand, the high cash flow leads to a reduction of the balance. The interest rate $r_{t}^{C}\left(M_{t-1}\right)$ is chosen so that the evolution of the balance $M_{t}$ is consistent with the evolution of the agent's continuation payoffs in the high state under the contract $\sigma^{*}$.

Since the balance on the credit line tracks the agent's continuation payoff in the high state, the agent has no incentive to divert excess cash flows. In the low state, there is no excess cash flow to divert. However, the credit limit in the low state is too generous compared to the agent's continuation payoff. If allowed, the agent would draw the credit line up to the limit, use all the borrowed cash to issue dividends and declare bankruptcy afterwards. To avoid this scenario, the credit line has the covenant that does not allow the agent to draw on the credit line to pay dividends.

\subsection{The Optimal Interest Rate Structure}

We now examine properties of the optimal interest rate structure. The dependence of the interest rate $r_{t}^{C}$ on the balance $M_{t-1}$ is expressed through the parameter $\tau$. Equations (38) and (39) should be read as follows: First, for a given balance $M_{t-1}$, we find $\tau$ that solves equation (38). Then, we substitute $\tau$ into equation (39) that gives us the interest rate $r_{t}^{C}\left(M_{t-1}\right)$ charged on the balance.

It is more convenient to examine the interest rate structure in the stationary setting with $T \rightarrow \infty$. Let

$$
V_{\infty}(y) \equiv E\left[\sum_{k=1}^{\infty} \beta^{k}\left(Y_{k}-y_{L}\right) \mid Y_{0}=y\right] .
$$

We can omit the time index and simplify equations (38) and (39) that become

$$
\left.M_{\tau}=V_{\infty}\left(y_{H}\right)-V_{\tau}\left(y_{H}\right)\right)
$$

and

$$
r^{C}\left(M_{\tau}\right)=\frac{V_{\tau}\left(y_{H}\right)-V_{\tau-1}\left(y_{H}\right)}{M_{\tau}} .
$$

We start our analysis of the optimal interest rate structure with two benchmark cases: independent cash flows and perfectly correlated cash flows.

Theorem 3 When the cash flows are independent over time $\left(Q\left(y_{L}\right)=Q\left(y_{H}\right)\right)$ or perfectly correlated $\left(Q\left(y_{L}\right)=0, Q\left(y_{H}\right)=1\right)$, the optimal interest rate is equal to the risk free interest 
rate: $r^{C}(M)=r$.

Proof. For ease of presentation, we prove the statement of Theorem 3 only for the case when the earliest default time $\tau$ is an integer. The reader can verify that the statement of Theorem 3 holds for any real $\tau$.

For the earliest default times $\bar{\tau}=1,2,3 \ldots$, equations (42) and (43) can be further simplified. In particular, the interest rate $r^{C}$ can be represented as ${ }^{7}$

$$
r^{C}\left(M_{\bar{\tau}}\right)=\beta^{\bar{\tau}} \frac{E\left[\left(Y_{\bar{\tau}}-y_{L}\right) \mid Y_{0}=y_{H}\right]}{M_{\bar{\tau}}},
$$

where, the balance $M_{\bar{\tau}}$ on the credit line is equal to a time zero value of the excess cash flows that will be generated after date $\bar{\tau}$ :

$$
M_{\bar{\tau}}=\beta^{\bar{\tau}} \sum_{k=1}^{\infty} \beta^{k} E\left[\left(Y_{\bar{\tau}+k}-y_{L}\right) \mid Y_{0}=y_{H}\right]
$$

When the cash flows are either independent or perfectly correlated, the conditional expectations of future excess cash flows are equal to the unconditional expectation:

$$
E\left[\left(Y_{\bar{\tau}+k}-y_{L}\right) \mid Y_{0}=y_{H}\right] \equiv Q\left(y_{H}\right)\left(y_{H}-y_{L}\right)
$$

Let $\mu \equiv Q\left(y_{H}\right)\left(y_{H}-y_{L}\right)$. Then,

$$
M_{\bar{\tau}}=\beta^{\bar{\tau}} \sum_{k=1}^{\infty} \beta^{k} \mu=\beta^{\bar{\tau}} \frac{\beta}{1-\beta} \mu .
$$

Substituting, (46) into (44) gives

$$
{ }_{r}^{C}\left(M_{\bar{\tau}}\right)=\frac{1-\beta}{\beta}=r .
$$

Theorem 3 replicates the result by DeMarzo and Fishman (2003) who consider independent cash flows. The reason the optimal interest rate is constant when the cash flows are i.i.d. is that the expectation of the future cash flows is constant.

The correlation of the cash flows introduces a link between the current cash flow and the future cash flows. This link becomes weaker as the time separating the current and future cash flows increases. This means that $E\left[\left(Y_{\bar{\tau}}-y_{L}\right) \mid Y_{0}=y_{H}\right]$, the numerator in (44),

\footnotetext{
${ }^{7}$ When the earliest default time $\tau$ is not an integer, the optimal interest rate is given by

$$
r^{C}\left(M_{\tau}\right)=\beta^{n_{\tau}} \frac{\left(1-l_{\tau}\right) E\left[\left(Y_{n_{\tau}}-y_{L}\right) \mid Y_{0}=y_{H}\right]+l_{\tau} E\left[\beta\left(Y_{n_{\tau}+1}-y_{L}\right) \mid Y_{0}=y_{H}\right]}{M_{\tau}}
$$
}


is decreasing ${ }^{8}$ with $\bar{\tau}$. Although $M_{\bar{\tau}} / \beta^{\bar{\tau}}$ also depends on $\bar{\tau}$, the overall effect on the interest rate $r^{C}$ is that $r^{C}$ is decreasing with $\bar{\tau}$, which means that $r^{C}$ is increasing with the balance on the credit line:

Theorem 4 The interest rate $r^{C}$ charged on the credit line is increasing with the outstanding balance $M$. Moreover

$$
\lim _{M \rightarrow 0} r^{C}(M)=r
$$

and

$$
r^{C}\left(C^{L}\right) \leq r \frac{Q\left(y_{H}\right)}{Q\left(y_{L}\right)}\left(1-\left(Q\left(y_{H}\right)-Q\left(y_{L}\right)\right)\right) .
$$

Proof. Consider the case when the earliest default time is an integer. According to (44) and (45), the interest rate on the credit line is given by

$$
\begin{aligned}
r^{C}\left(M_{\bar{\tau}}\right) & =\beta^{\bar{\tau}} \frac{E\left[\left(Y_{\bar{\tau}}-y_{L}\right) \mid Y_{0}=y_{H}\right]}{\beta^{\bar{\tau}} \sum_{k=1}^{\infty} \beta^{k} E\left[\left(Y_{\bar{\tau}+k}-y_{L}\right) \mid Y_{0}=y_{H}\right]} \\
& =\left(\sum_{k=1}^{\infty} \beta^{k} \frac{E\left[\left(Y_{\bar{\tau}+k}-y_{L}\right) \mid Y_{0}=y_{H}\right]}{E\left[\left(Y_{\bar{\tau}}-y_{L}\right) \mid Y_{0}=y_{H}\right]}\right)^{-1},
\end{aligned}
$$

for the earliest default time $\bar{\tau}=1,2,3 \ldots$

According to Lemma 5(iii) in Appendix,

$$
\frac{E\left[\left(Y_{\bar{\tau}+k}-y_{L}\right) \mid Y_{0}=y_{H}\right]}{E\left[\left(Y_{\bar{\tau}}-y_{L}\right) \mid Y_{0}=y_{H}\right]} \leq \frac{E\left[\left(Y_{\bar{\tau}+1+k}-y_{L}\right) \mid Y_{0}=y_{H}\right]}{E\left[\left(Y_{\bar{\tau}+1}-y_{L}\right) \mid Y_{0}=y_{H}\right]}
$$

The last inequality means that if we increase the earliest default time by one, then every term in the sum in (48) will become bigger, and the interest rate will therefore become smaller. The higher the earliest default time $\bar{\tau}$, the smaller the balance $M_{\bar{\tau}}$ on the credit line is. Thus, the interest rate charged on the credit line increases with the balance on the credit line, for the earliest default time taking integer values. One can verify that this result holds for any earliest default time.

When $\bar{\tau} \rightarrow \infty, M_{\bar{\tau}} \rightarrow 0$. The cash flow process is asymptotically stationary. As a result,

$$
\lim _{\bar{\tau} \rightarrow \infty} \frac{E\left[\left(Y_{\bar{\tau}+k}-y_{L}\right) \mid Y_{0}=y_{H}\right]}{E\left[\left(Y_{\bar{\tau}}-y_{L}\right) \mid Y_{0}=y_{H}\right]}=1
$$

\footnotetext{
${ }^{8}$ See Lemma 5 in Appendix.
} 
Using (48),

$$
\begin{aligned}
\lim _{M \rightarrow 0} r^{C}(M) & =\left(\sum_{k=1}^{\infty} \beta^{k}\right)^{-1} \\
& =\frac{1-\beta}{\beta}=r .
\end{aligned}
$$

The interest rate charged on the credit line is the highest when the balance on the credit line reaches the credit limit, at which point the earliest default time $\tau=1$. Using (48) the fact ${ }^{9}$ that

$$
\begin{aligned}
E\left[\left(Y_{k}-y_{L}\right) \mid Y_{0}\right. & \left.=y_{H}\right] \geq \lim _{s \rightarrow \infty} E\left[\left(Y_{s}-y_{L}\right) \mid Y_{0}=y_{H}\right] \\
& =\frac{Q\left(y_{L}\right)}{1-\left(Q\left(y_{H}\right)-Q\left(y_{L}\right)\right)}\left(y_{H}-y_{L}\right),
\end{aligned}
$$

we can write that

$$
\begin{aligned}
r^{C}\left(M_{1}\right) & \leq \frac{E\left[\left(Y_{1}-y_{L}\right) \mid Y_{0}=y_{H}\right]}{\sum_{k=1}^{\infty} \beta^{k} \frac{Q\left(y_{L}\right)}{1-\left(Q\left(y_{H}\right)-Q\left(y_{L}\right)\right)}\left(y_{H}-y_{L}\right)} \\
& =\frac{1-\beta}{\beta} \frac{Q\left(y_{H}\right)}{Q\left(y_{L}\right)}\left(1-\left(Q\left(y_{H}\right)-Q\left(y_{L}\right)\right)\right) \\
& =r \frac{Q\left(y_{H}\right)}{Q\left(y_{L}\right)}\left(1-\left(Q\left(y_{H}\right)-Q\left(y_{L}\right)\right)\right) .
\end{aligned}
$$

Theorem 4 says that the optimal interest rate schedule starts with the risk-free interest rate at the zero balance and monotonically increases with the balance. The interest rate, however, never goes above the boundary, given by (47). When cash flows are independent over time or perfectly correlated, the optimal interest rate is equal to the risk free interest rate. Thus, the correlation of the cash flows explains the fact that the optimal interest rate is increasing with the balance.

In order to understand the optimal interest rate structure, it is useful to reexamine the properties of the optimal contract. Under the optimal contract, the agent is always indifferent between using excess cash flows to pay down the credit line or diverting them for his own consumption. On the equilibrium path, the agent who holds fraction $\lambda$ of the equity uses excess cash flows to pay off the credit line first and then to issue dividends. His continuation payoff in equilibrium is thus equal to the expected value of his share of the future dividends. Instead of paying down the credit line, the agent, however, can get the same continuation payoff by diverting all the future excess cash flows until he exhausts the credit line, at

\footnotetext{
${ }^{9}$ See Lemma 5(iv) in Appendix.
} 
which point he defaults. The optimal interest rate minimizes expected costs of default, while discouraging cash flow diversion by the agent. Holding the credit limit constant, an interest rate schedule, which lies below the optimal schedule, would not be incentive competitive. On the other hand, an interest rate schedule, which lies above the optimal schedule, would result in unnecessary high chances of default.

After diverting an excess cash flow, the agent is in a better position when the cash flows are positively correlated, than when they are independent over time. Indeed, subsequent cash flows are more likely to be high, given the high cash flow in the current period. Hence, given the same time interval, the agent is capable of stealing on average more cash when the cash flows are positively correlated. Under the optimal contract, a higher interest rate charged on the credit line precipitates default and discourages the agent from stealing cash flows.

The optimal interest rate structure reflects an average rate at which the agent can divert the cash flows. When the cash flows are positively correlated, the expectation of a future cash flow depends on the current cash flow as well as the time between the current date and the date of the future cash flow realization. Figure 8 presents an example of the expected amount of cash the agent can divert if he starts diverting cash flows in the high state. One can see that because the cash flows are positively correlated the expected value of the cash flows decreases as the times goes on. This fact has an important implication for the agent's willingness to divert the cash flows. The higher balance on the credit line, the shorter time interval during which the agent can divert the cash flows. However, as Figure 8 illustrates, the amount of cash that the agent can divert per period is higher, the shorter the time interval during which the diversion takes place. Thus, the agent has a stronger motive to divert cash flows when the balance is high. In order to prevent stealing, the time interval during which the agent is allowed to run the project when he reports the low cash flow in each period should be shorten. Under the optimal contract, this is done by charging higher interest rate at the end of the credit line.

When deriving the optimal contract, we assumed that the agent is not allowed to save and therefore cannot overreport cash flows. Given the result of Theorem 4, it is easy to see that optimal contract remains incentive compatible even when the agent is allowed to save. The interest rate $r^{C}$ charged on the balance on the credit line is higher than the rate $\rho$, at which the agent's savings grow. Therefore, if the agent diverts the high cash flow today, saves it privately and and then uses the savings to pay down the credit line in the future, the resulting balance on the credit line will be higher than when he uses all the cash flows to pay down the credit line immediately. Thus, the agent has no incentives to save under the optimal contract. 


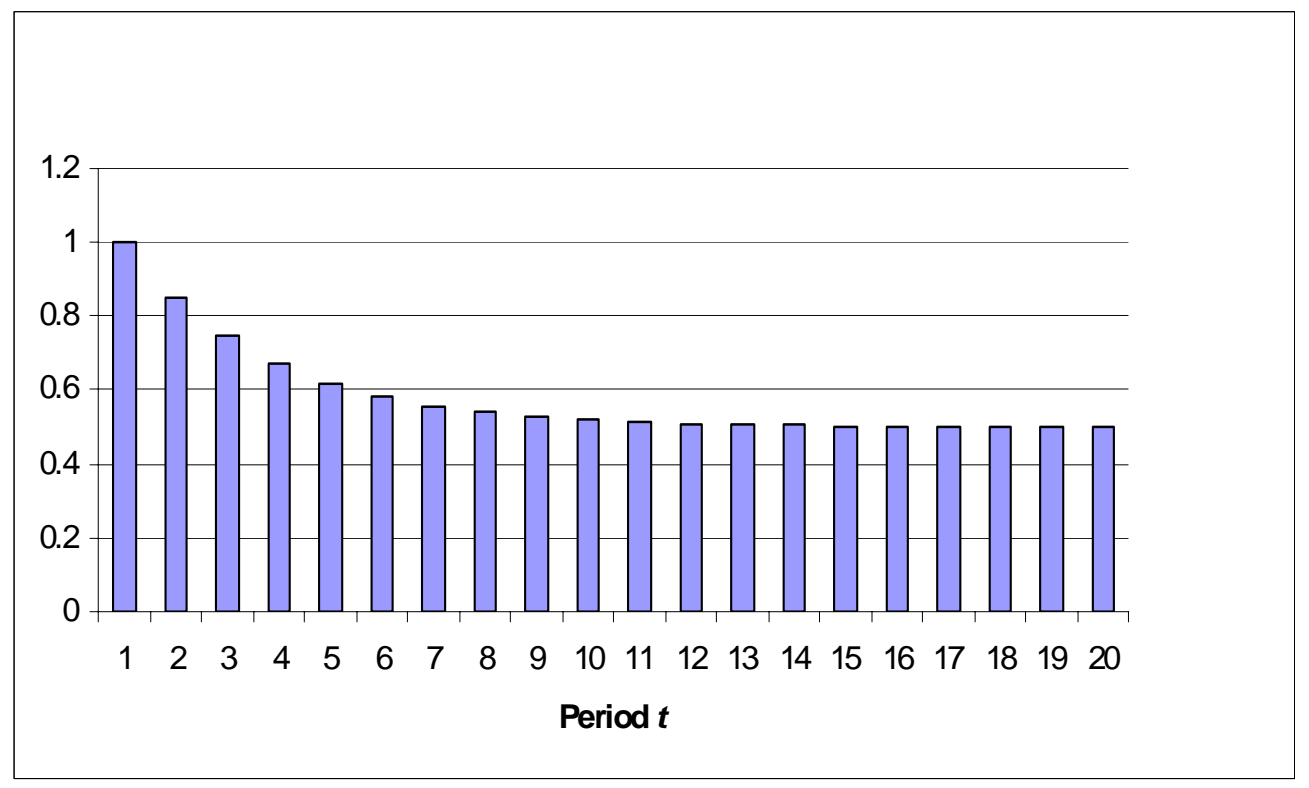

Figure 8: Expectation of future cash flows, given the high cash flow in period $1, E\left[Y_{t} \mid Y_{1}=y_{H}\right]$, for parameters $y_{L}=0, y_{H}=1, Q\left(y_{L}\right)=0.15, Q\left(y_{H}\right)=0.85$

\subsubsection{An Example}

Now, we illustrate how the optimal interest rate schedule changes with the degree of the cash flow correlation. Consider a stationary case with the risk-free interest rate $5 \%(r=0.05)$, and symmetric transition probabilities: $Q\left(y_{H}\right)=q$ and $Q\left(y_{L}\right)=1-q$, where $q \in[0.5,1]$. Higher $q$ means stronger correlation. When $q=0.5$, the cash flows are independent over time. When $q=1$, the cash flows are perfectly correlated.

As illustrated in Figure 9, the interest rate $r^{C}$ on the credit line is equal to the risk-free interest rate when $q=0.5$ for any size of the outstanding balance $M$. When $q$ is increased to 0.6 , the optimal interest rate $r^{C}$ lies above the risk free rate $r$. However, the difference between $r^{C}$ and $r$ remains insignificant as long as the balance on the credit line stays below $86 \%$ of the credit limit. The interest rate grows sharply once the balance becomes greater than 86 percentage points of the credit limit, and almost reaches $6 \%$ when the credit line is completely exhausted. A greater degree of the cash flow correlation causes the interest rate to escalate earlier. For $q=0.85$, the interest rate $r^{C}$ becomes visibly greater than $r$ when the balance crosses the $40 \%$ mark. Afterwards, $r^{C}$ keeps growing and reaches $7.9 \%$ in the end. When the cash flows are extremely correlated $(q=0.995)$, the interest rate jumps sharply at the beginning and remains relatively flat afterwards, staying just below 0.055 . In the deterministic limit $(q=1)$, the interest rate remains identically equal to the risk-free interest rate, as was the case with the independent cash flows. 


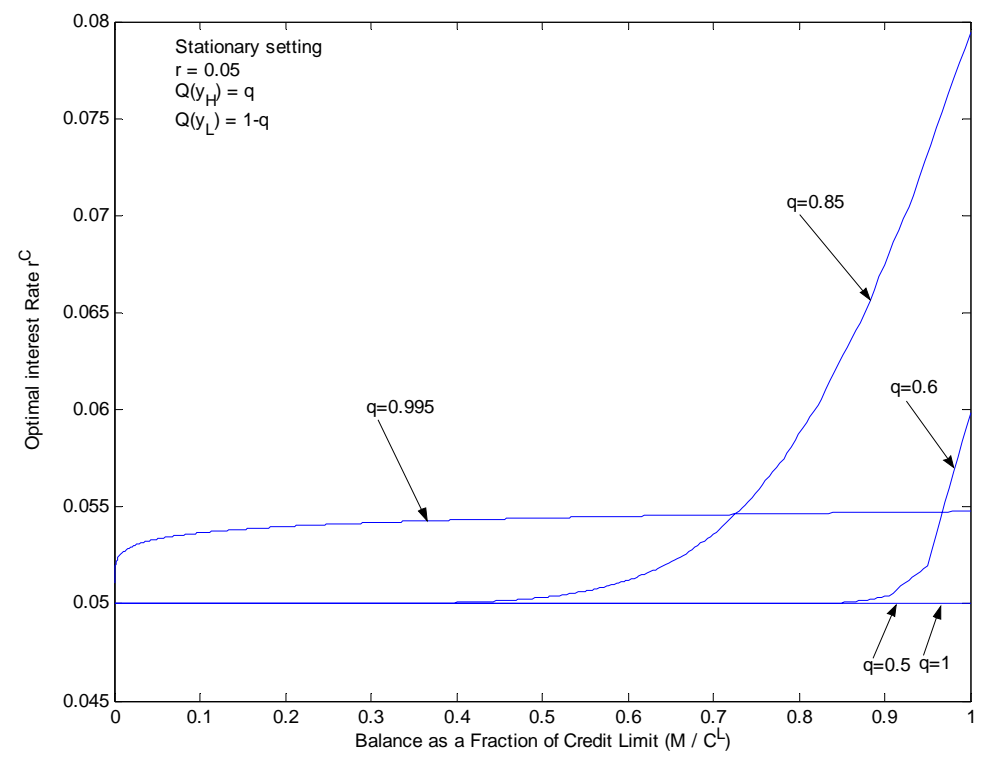

Figure 9: The optimal interest rate structure.

\subsection{The Market Values of the Securities}

The continuation payoffs for the agent and the investor given by $a_{t}^{d}\left(y_{t}\right)$ and $b_{t}^{d}\left(y_{t}, a_{t}^{d}\left(y_{t}\right)\right)$ can be used to determine market values of the securities used in the implementation of the optimal contract. For ease of presentation we again consider stationary setting with $T=\infty$. We will refer to the combination of the bond and the credit line balance as debt. Let $D(y, M)$ and $S(y, M)$ denote the market value of the debt and the equity, given the state $y$ and the balance $M$ on the credit line. Since the agent owns the fraction $\lambda$ of the equity and has no other securities, the agent's continuation payoff is given by

$$
a_{t}^{d}\left(y_{t}\right)=\lambda S\left(y_{t}, M_{t}\right) .
$$

Equation (41) connects the agent's continuation payoff in the high state to the balance on the credit line. We can rewrite (41) as follows

$$
a_{t}^{d}\left(y_{H}\right)=\lambda\left(C^{L}-M_{t}+V_{1}\left(y_{H}\right)\right) .
$$

Thus, the value of the equity in the high state is given by

$$
S\left(y_{H}, M_{t}\right)=C_{t}^{L}-M_{t}+V_{1}\left(y_{H}\right) .
$$


The agent's continuation payoff in the low state that corresponds to the same earliest default time and hence to the same balance on the credit line is $a_{t}^{d}\left(y_{L}\right)=c^{-1}\left(a_{t}^{d}\left(y_{H}\right)\right)$. Hence, the equity value in the low state is given by

$$
S\left(y_{L}, M_{t}\right)=\frac{1}{\lambda} c^{-1}\left(\lambda\left(C_{t}^{L}-M_{t}+V_{1}\left(y_{H}\right)\right)\right) .
$$

Note that while $S\left(y_{H}, M_{t}\right)$ is linear in $M_{t}, S\left(y_{L}, M_{t}\right)$ is convex in $M_{t}$.

The investor's continuation payoff $b^{d}\left(y_{t}, a_{t}^{d}\left(y_{t}\right)\right)$ consists of the value of the debt plus the value of the fraction $(1-\lambda)$ of the equity. Thus, the value of the debt in the high state is given by

$$
D\left(y_{H}, M_{t}\right)=b^{d}\left(y_{H}, \lambda\left(C^{L}-M_{t}+V_{1}\left(y_{H}\right)\right)\right)-(1-\lambda)\left(C^{L}-M_{t}+V_{1}\left(y_{H}\right)\right),
$$

and the value of the debt in the low state is given by

$$
D\left(y_{L}, M_{t}\right)=b^{d}\left(y_{L}, c^{-1}\left(\lambda\left(C^{L}-M_{t}+V_{1}\left(y_{H}\right)\right)\right)\right)-\frac{(1-\lambda)}{\lambda} c^{-1}\left(\lambda\left(C^{L}-M_{t}+V_{1}\left(y_{H}\right)\right)\right) .
$$

Zero balance on the credit line corresponds to the first best. Once the first best is reached the low cash flows are paid to the investor as the bond's coupon payments, while the excess cash flows are used to pay dividends to the equity holders. The value of the debt in this situation is equal to the value of the future low cash flows:

$$
D\left(y_{t}, 0\right)=\frac{\beta y_{L}}{1-\beta} .
$$

Assuming that the bond is risk-free ${ }^{10}, D\left(y_{t}, 0\right)$ is the nominal as well as the market value of the bond. Differentiating (51) with respect to $M_{t}$ gives

$$
\frac{\partial D\left(y_{H}, M_{t}\right)}{\partial M_{t}}=1-\lambda\left(1+\frac{\partial b^{d}\left(y_{H}, \lambda\left(C^{L}-M_{t}+V_{1}\left(y_{H}\right)\right)\right)}{\partial a_{t}^{d}}\right) \leq 1 .
$$

The inequality follows from the fact that the derivative of the value function $b^{d}$ is greater than or equal to -1 . This leads us to the following theorem:

Theorem 5 The market value of the credit line debt is below its nominal value.

For the high state, the result of Theorem 5 follows from (52). For the low state, the result of Theorem 5 follows from the fact that the value of debt in the low state is below that in the high state.

\footnotetext{
${ }^{10}$ Since the liquidation value of the project is greater or equal than $\frac{\beta y_{L}}{1-\beta}$, the bond is risk free if it has seniority over the credit line. The seniority however does not matter since the investor holds both the bond and the credit line. So, we can assume without loss of generality that the bond is risk free.
} 


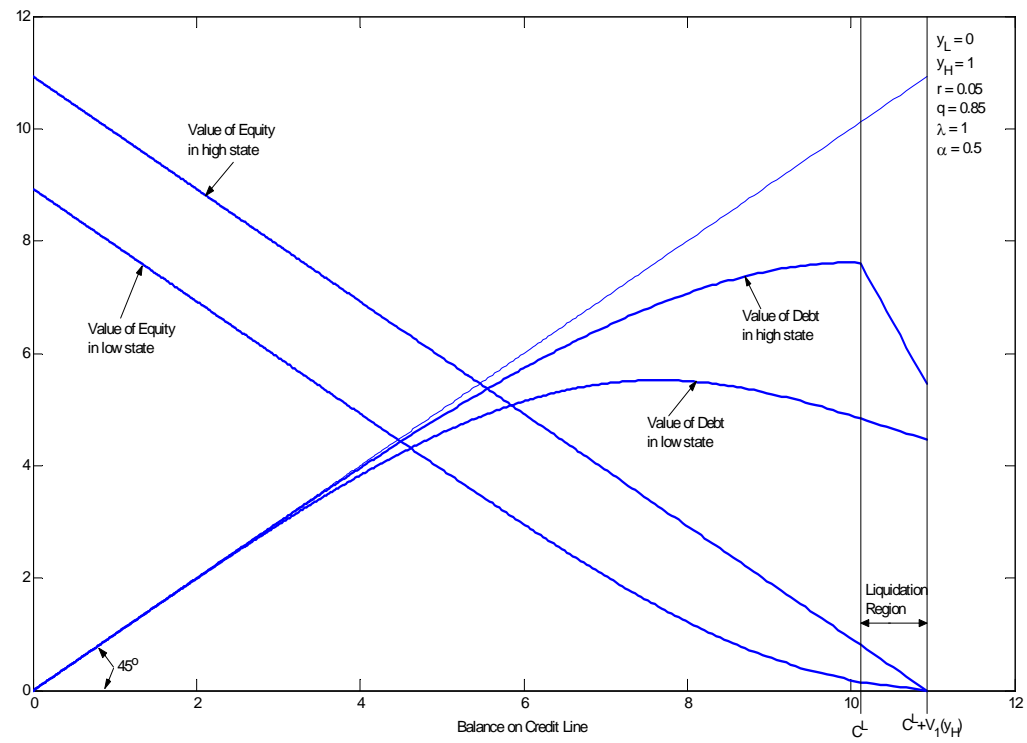

Figure 10: Values of securities.

Theorem 5 implies that although the interest rate on the credit line increases as the balance on the credit line goes up, it is not enough to compensate the investor for the higher risk. Thus, the prediction of the agency theory of performance pricing developed in this paper is in contrast to the theory that says that performance pricing is used to compensate investors for higher risks in times of poor performance. This prediction opens room for an empirical test of the theories. Another empirically testable implication of Theorem 5 is that in order to break-even the investor must charge an additional fee for activating the credit line. In our setting, this means that the initial balance on the credit line is greater than the amount of money the agent actually borrows from the credit line at time zero.

Figure 10 plots the values of the equity and the credit line debt for the following parameters: $y_{L}=0, y_{H}=1, r=0.05, Q\left(y_{H}\right)=q=0.85, Q\left(y_{L}\right)=1-q, \lambda=1, \alpha=0.5$. Given these parameters, there is no bond, the agent holds $100 \%$ of the equity, and the debt value is equal to the value of the balance on the credit line. As Theorem 5 predicts the values of the credit line debt in both states lie below the 45 degree line.

Since the value of the debt in the low state is downward sloping when the balance is close to the credit limit, the contract is not renegotiation proof. By writing off a part of the credit line debt, the investor can increase the continuation payoffs for himself and for the agent. A possibility of such renegotiation would destroy the agent's incentives to pay off the credit line. Thus, the full commitment to the terms of the contract is crucial in this example. It is not always the case that the contract is not renegotiation proof. An important parameter 


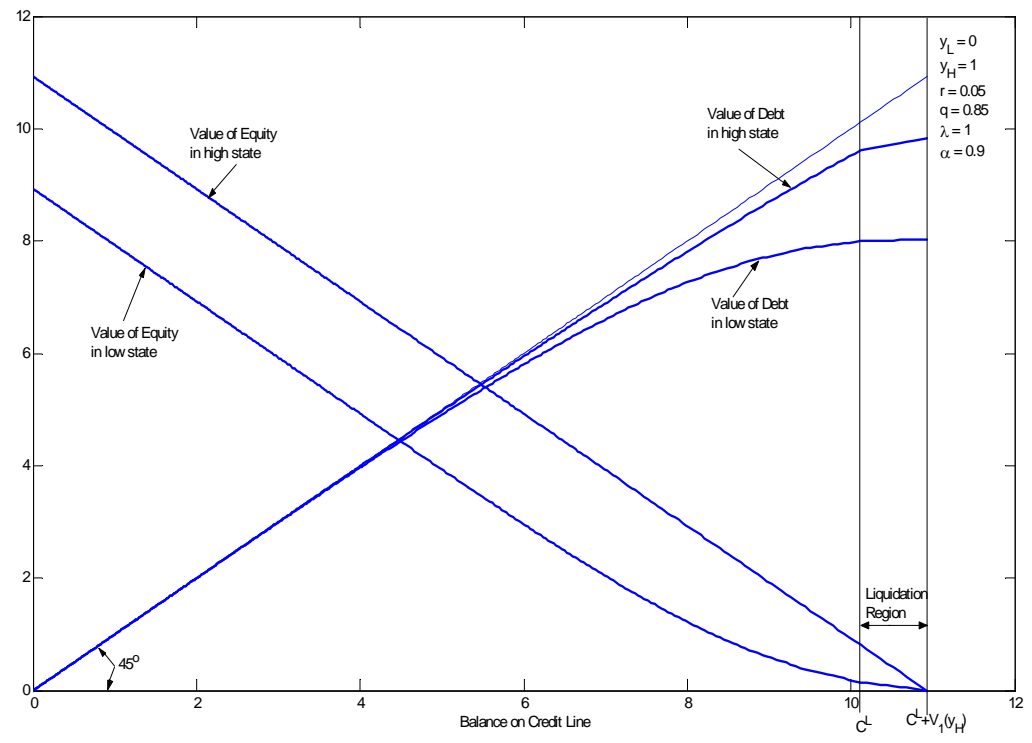

Figure 11: Renegotiation proof contract

for the renegotiation proofness is the liquidation value of the project. The example in Figure 10 has $\alpha=0.5$. As Figure 11 shows, the optimal contract becomes renegotiation proof when $\alpha=0.9$.

\section{The Continuous Time Model}

In this section we take a continuous time limit of our discrete time model. We do it by decreasing the length of each period to zero and adjusting the transition probabilities at the same time. We then characterize the optimal contract in continuous time. Unlike the discrete time setting, the optimal interest rate in the continuous time setting is given by a smooth function, the continuation functions can be easily obtained by solving a pair of differential equations, and the optimal liquidation policy is deterministic.

\subsection{The Continuous Time Setting}

In this section, we consider the stationary setting, i.e. $T \rightarrow \infty$. Letting $\Delta$ denote the length of one period, the discount factor between periods is given by $\beta=e^{-r \Delta}$, where $r$ is the riskfree interest rate. For ease of presentation, we normalize the low cash flow per unit of time to zero, $y_{L}=0$, and the high cash flow to one, $y_{H}=1$. The cash flow in period $t$ is proportional to the length of the period and is given by $\Delta Y_{t}$, where $Y_{t} \in\{0,1\}$. In addition, we assume that the cash flows follow a symmetric Markov process, with the transition probabilities given 


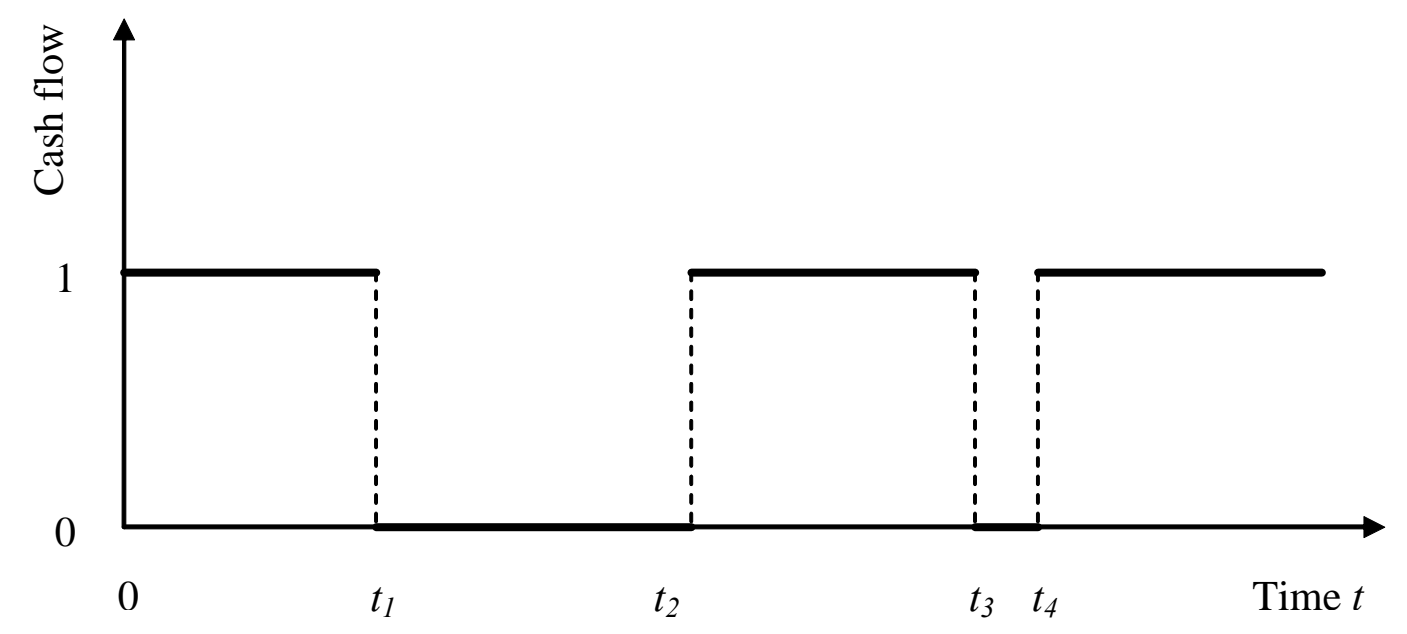

Figure 12: Continuous time cash flow process.

by $Q\left(y_{H}\right)=q$ and $Q\left(y_{L}\right)=1-q$, where

$$
q=e^{-\gamma \Delta} .
$$

The probability $q$ that the cash flow remains the same as in the previous period is adjusted to reflect the length of the period. In the limit, as $\Delta \rightarrow 0$, the discrete time Markov chain becomes a continuous time switching process with the parameter $\gamma \in[0, \infty)$ being the intensity of switching from one state to another. Indeed, since $q=e^{-\gamma \Delta} \approx 1-\gamma \Delta$,

$$
\gamma=\lim _{\Delta \rightarrow 0} \frac{1-q}{\Delta}
$$

Higher $\gamma$ means higher intensity of switching, or smaller degree of the correlation. The cash flows are perfectly correlated when $\gamma=0$, and become independent over time when $\gamma \rightarrow+\infty$.

Figure 12 shows a possible cash flow path in continuous time. The initial cash flow is high. At time $t_{1}$, the cash flow switches to the low state, where it stays until time $t_{2}$. Then, it switches back to the high state, and then switches again to the low state at time $t_{3}$.

\subsection{Characterization of the Optimal Continuous Contract}

Since the evolution of the agent's continuation payoffs under the optimal contract is characterized by (31)-(34) in terms of the evolution of the earliest default time, we start our analysis with examining properties of the continuous time version of the functions $V_{\tau}$. The expected present value of the cash flows that the project will generate during the time interval $[t, t+\tau]$ is given by $E\left[\sum_{k=1}^{\tau / \Delta} e^{-r \Delta k} \Delta Y_{t+k} \mid Y_{t}=y\right]$, where $\tau / \Delta$ is approximately equal to the number 
of periods in the interval $[t, t+\tau]$. Although $\tau / \Delta$ may not be a whole number, the remainder does not matter in the limit. Thus, we define $\tilde{V}_{\tau}$ in the continuous time setting as follows:

$$
\tilde{V}_{\tau}(y) \equiv \lim _{\Delta \rightarrow 0} E\left[\sum_{k=1}^{\tau / \Delta} e^{-r \Delta k} \Delta Y_{t+k} \mid Y_{t}=y\right]
$$

Lemma 4 The expected present value of the cash flows that the project generates during the time $\tau$ is given by

$$
\tilde{V}_{\tau}(y)=\frac{1-e^{-r \tau}}{2 r}+(2 y-1) \frac{1-e^{-(2 \gamma+r) \tau}}{2(2 \gamma+r)}
$$

The proof is given in the appendix.

The first term in (53) is independent of the initial state $y$ and is equal to the expected present value of the cash flows that the project generates during the time $\tau$ when cash flows are independent over time, i.e., $\gamma=+\infty$. The second term is positive when the initial state is high, and negative when the initial state is low. The value of the project that operates indefinitely long is denoted by

$$
\tilde{V}_{\infty}(y) \equiv \frac{1}{2 r}+(2 y-1) \frac{1}{2(2 \gamma+r)} .
$$

$\tilde{V}_{\infty}(y)$ is the dividend boundary. The agent gets paid under the optimal contract only when his continuation payoff exceeds the dividend boundary.

Since the continuation functions now are no longer dependent on time in the stationary setting, we can suppress the time subscript. We can also get rid of superscripts and consider a single continuation function instead of the three different continuation functions $b_{t}^{y}, b_{t}^{d}, b_{t}^{e}$ that we had in the discrete time model for each period. Since the agent's payoffs under an optimal continuation contract depend on the current state, but can be characterized in terms of the earliest default time $\tau$, which does not depend on the current state, it is convenient to express the continuation payoff for the investor as a function of the earliest default time $\tau$. We define

$$
\begin{aligned}
b_{L}(\tau) & \equiv \lim _{\Delta \rightarrow 0} b^{d}\left(y_{L}, \lambda V_{\tau}\left(y_{L}\right)\right) \\
b_{H}(\tau) & \equiv \lim _{\Delta \rightarrow 0} b^{d}\left(y_{H}, \lambda V_{\tau}\left(y_{H}\right)\right)
\end{aligned}
$$

An advantage of having the earliest default time $\tau$, instead of the agent's continuation payoff $a$, as the argument of the continuation functions $b_{L}$ and $b_{H}$ is that the agent's continuation payoff jumps every time the cash flow changes, while the earliest default time does not. Because of the continuity of the earliest default time process, we can characterize the continuation functions $b_{L}$ and $b_{H}$ using a pair of differential equations. Let $b_{L}^{\prime}$ and $b_{H}^{\prime}$ denote the derivatives of the continuation functions in continuous time. 
Proposition 10 In the continuous time setting, the liquidation boundary $a^{L}(y)=0$. The earliest default time evolves as follows

$$
d \tau=\left(-1+\frac{2 Y_{t}}{e^{-r \tau}+e^{-(2 \gamma+r) \tau}}\right) d t
$$

The continuation functions $b_{L}$ and $b_{H}$ solve the pair of the following differential equations:

$$
\begin{aligned}
r b_{L}(\tau) & =\gamma\left(b_{H}(\tau)-b_{L}(\tau)\right)-b_{L}^{\prime}(\tau) \\
r b_{H}(\tau) & =1-\gamma\left(b_{H}(\tau)-b_{L}(\tau)\right)+\frac{2-e^{-r \tau}-e^{-(2 \gamma+r) \tau}}{e^{-r \tau}+e^{-(2 \gamma+r) \tau}} b_{H}^{\prime}(\tau)
\end{aligned}
$$

Moreover,

$$
\begin{aligned}
\lim _{\tau \rightarrow \infty} b_{L}(\tau) & =(1-\lambda) \tilde{V}_{\infty}\left(y_{L}\right) \\
\lim _{\tau \rightarrow \infty} b_{H}(\tau) & =(1-\lambda) \tilde{V}_{\infty}\left(y_{H}\right) \\
\lim _{\tau \rightarrow 0} b_{L}(\tau) & =\alpha \tilde{V}_{\infty}\left(y_{L}\right)
\end{aligned}
$$

Proof. The default boundary in the discrete time setting is given by $a^{L}(y)=\lambda E\left[\Delta Y_{t+1} \mid Y_{t}=y\right]$. As $\Delta \rightarrow 0, a^{L}(y) \rightarrow 0$.

In discrete time, if the agent's continuation payoff at time $t$ is equal to $\lambda V_{\tau}\left(Y_{t}\right)$, his continuation payoff at time $t+\Delta$, according to (31)-(34), is given by $V_{\tau+d \tau}=\lambda V_{\tau-\Delta}\left(Y_{t+\Delta}\right)+$ $\lambda \Delta Y_{t+\Delta}$, where $d \tau$ denotes the increment of the earliest default time. Using the Taylor expansion, we obtain

$$
d \tau=\left(-1+\frac{Y_{t+\Delta}}{\frac{\partial V_{\tau}\left(Y_{t+\Delta}\right)}{\partial \tau}}\right) \Delta
$$

Since $\frac{\partial \tilde{V}_{\tau}\left(Y_{t}\right)}{\partial \tau}=0.5\left(e^{-r \tau}+\left(2 Y_{t}-1\right) e^{-(2 \gamma+r) \tau}\right)$, taking the limit and replacing $\Delta$ by $d t$ yields (54).

The probability that $Y_{t+\Delta}=y_{L}$ is equal to $e^{-\gamma \Delta}$. Letting $b^{d}$ denote the middle-of-period continuation function in the discrete time setting, we can write

$$
\begin{aligned}
b^{d}\left(y_{L}, \lambda V_{\tau}\left(y_{L}\right)\right)= & e^{-r \Delta}\left(\left(1-e^{-\gamma \Delta}\right)\left(\Delta+b^{d}\left(y_{H}, \lambda V_{\tau-\Delta}\left(y_{H}\right)+\lambda \Delta\right)\right)\right) \\
& +e^{-r \Delta} e^{-\gamma \Delta} b^{d}\left(y_{L}, \lambda V_{\tau-\Delta}\left(y_{L}\right)\right) .
\end{aligned}
$$

Using Taylor expansions, we obtain

$$
\begin{aligned}
b^{d}\left(y_{L}, \lambda V_{\tau}\left(y_{L}\right)\right) \approx & (1-r \Delta)\left(\gamma \Delta\left(\Delta+b^{d}\left(y_{H}, \lambda V_{\tau-\Delta}\left(y_{H}\right)+\lambda \Delta\right)\right)\right. \\
& \left.+(1-\gamma \Delta) b^{d}\left(y_{L}, \lambda V_{\tau-\Delta}\left(y_{L}\right)\right)\right) .
\end{aligned}
$$


Collecting the terms in (60) and ignoring those that are of order $\Delta^{2}$ or smaller yields

$$
\begin{aligned}
& \frac{b^{d}\left(y_{L}, \lambda V_{\tau}\left(y_{L}\right)\right)-b^{d}\left(y_{L}, \lambda V_{\tau-\Delta}\left(y_{L}\right)\right)}{\Delta} \\
\approx & \left.\gamma\left(b^{d}\left(y_{H}, \lambda V_{\tau-\Delta}\left(y_{H}\right)+\lambda \Delta\right)\right)-b^{d}\left(y_{L}, \lambda V_{\tau-\Delta}\left(y_{L}\right)\right)\right) \\
& -\gamma r b^{d}\left(y_{L}, \lambda V_{\tau-\Delta}\left(y_{L}\right)\right) .
\end{aligned}
$$

As $\Delta \rightarrow 0,(61)$ becomes (55).

Now, we consider the case with the high initial state. Similarly to (??), we can write

$$
\begin{aligned}
b^{d}\left(y_{H}, \lambda V_{\tau}\left(y_{H}\right)\right)= & e^{-r \Delta} e^{-\gamma \Delta}\left(\Delta+b^{d}\left(y_{H}, \lambda V_{\tau-\Delta}\left(y_{H}\right)+\lambda \Delta\right)\right) \\
& +e^{-r \Delta}\left(1-e^{-\gamma \Delta}\right) b^{d}\left(y_{L}, \lambda V_{\tau-\Delta}\left(y_{L}\right)\right) .
\end{aligned}
$$

Using the Taylor expansion and rearranging terms yields

$$
\begin{aligned}
& \frac{b^{d}\left(y_{H}, \lambda V_{\tau-\Delta}\left(y_{H}\right)+\lambda \Delta\right)-b^{d}\left(y_{H}, \lambda V_{\tau}\left(y_{H}\right)\right)}{\Delta} \\
\approx & -1+r b^{d}\left(y_{H}, \lambda V_{\tau-\Delta}\left(y_{H}\right)\right) \\
& \left.+\gamma\left(b^{d}\left(y_{H}, \lambda V_{\tau-\Delta}\left(y_{H}\right)+\lambda \Delta\right)\right)-b^{d}\left(y_{L}, \lambda V_{\tau-\Delta}\left(y_{L}\right)\right)\right) .
\end{aligned}
$$

Taking the limit of the left-hand-side of (63) gives

$$
\begin{aligned}
& \lim _{\Delta \rightarrow 0} \frac{b^{d}\left(y_{H}, \lambda V_{\tau-\Delta}\left(y_{H}\right)+\lambda \Delta\right)-b^{d}\left(y_{H}, \lambda V_{\tau}\left(y_{H}\right)\right)}{\Delta} \\
= & \lim _{\Delta \rightarrow 0} \frac{\partial b^{d}\left(y_{H}, \lambda V_{\tau-\Delta}\left(y_{H}\right)+\lambda \Delta\right)}{\partial a} \lambda\left(1-\frac{V_{\tau}\left(y_{H}\right)-V_{\tau-\Delta}\left(y_{H}\right)}{\Delta}\right) \\
= & \frac{\partial b^{d}\left(y_{H}, \lambda \tilde{V}_{\tau}\left(y_{H}\right)\right)}{\partial a} \lambda\left(1-\frac{\partial \tilde{V}_{\tau}\left(y_{H}\right)}{\partial \tau}\right) \\
= & \frac{b_{H}^{\prime}(\tau)}{\lambda \frac{\partial \tilde{V}_{\tau}\left(y_{H}\right)}{\partial \tau}} \lambda\left(1-\frac{\partial \tilde{V}_{\tau}\left(y_{H}\right)}{\partial \tau}\right) \\
= & b_{H}^{\prime}(\tau)\left(\left(\frac{\partial \tilde{V}_{\tau}\left(y_{H}\right)}{\partial \tau}\right)^{-1}-1\right) \\
= & b_{H}^{\prime}(\tau)\left(\frac{2}{e^{-r \tau}+e^{-(2 \gamma+r)}}-1\right) \\
= & b_{H}^{\prime}(\tau) \frac{2-e^{-r \tau}-e^{-(2 \gamma+r)}}{e^{-r \tau}+e^{-(2 \gamma+r)}}
\end{aligned}
$$

Here, we used the Taylor expansion for the function $b^{d}$, and then applied the formula for the derivative of a composite function to $\frac{\partial b^{d}\left(y_{H}, \lambda \tilde{V}_{\tau}\left(y_{H}\right)\right)}{\partial a}$. Taking the limit of the right-hand-side of (63) and substituting (64) into (63) yields (56). 


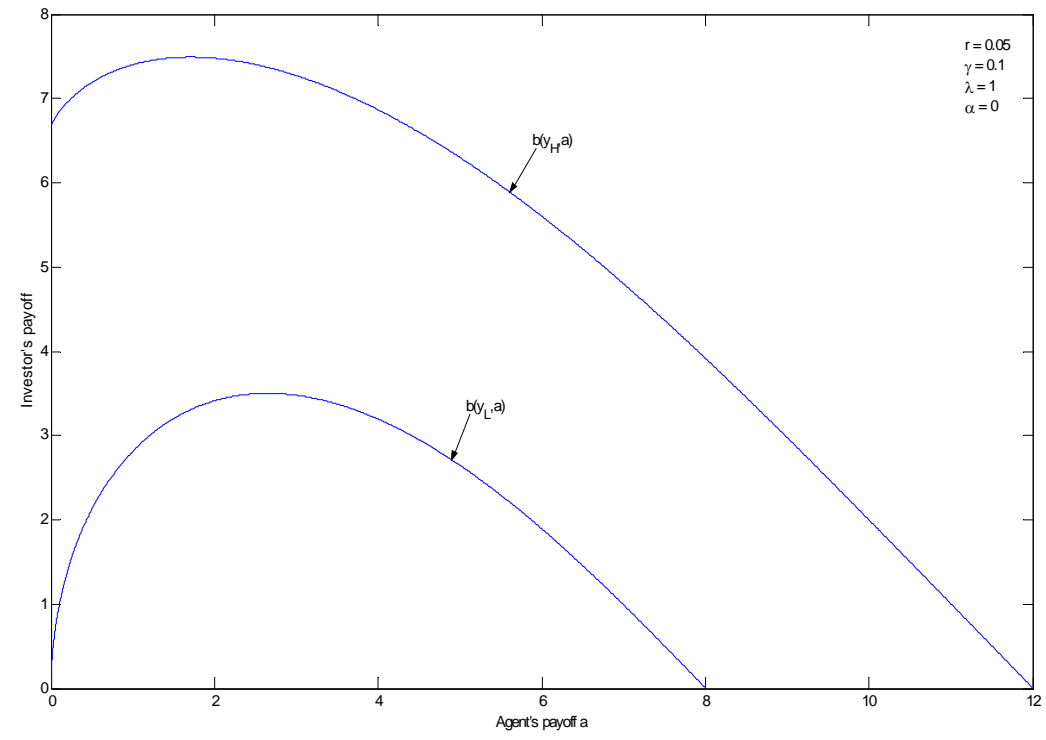

Figure 13: Continuation functions in continuous time.

When the agent's continuation payoff reaches the dividend boundary $V_{\infty}\left(y_{t}\right)$, the first best is implemented as follows. The agent consumes the fraction $\lambda$ of all subsequent cash flows, while the investor gets the fraction $(1-\lambda)$ of the subsequent cash flows. Hence

$$
b^{d}\left(\lambda V_{\infty}\left(y_{t}\right)\right)=(1-\lambda) V_{\infty}\left(y_{t}\right) .
$$

As $\Delta \rightarrow 0,(65)$ yields (57) and (58). Condition (59) follows from the fact that liquidation value of the project in the low state is given by $\alpha \tilde{V}_{\infty}\left(y_{L}\right)$.

We interpret equations (55) and (56) as follows. In order to get $b$, the investor must earn total return $r b$. Equation (55) says that in the low state the return is equal to the expected benefits from switching to the high state, $\gamma\left(b_{H}(\tau)-b_{L}(\tau)\right)$, less the derivative, $b_{L}^{\prime}(\tau)$, which reflects the fact that reporting the low cash flow is penalized by reducing the earliest default time. Equation (56) says that the return in the high state is equal to the high cash flow, 1 , less the expected loss from switching to the low state, $\gamma\left(b_{H}(\tau)-b_{L}(\tau)\right)$, plus the term $\frac{2-e^{-r \tau}-e^{-(2 \gamma+r) \tau}}{e^{-r \tau}+e^{-(2 \gamma+r) \tau}} b_{H}^{\prime}(\tau)$ that reflects an increase in the earliest default time when the high cash flow is reported. Proposition 10 also establishes that the optimal liquidation policy is no longer stochastic. Default happens exactly when the agent's continuation payoff reaches zero.

We can use differential equations (55) and (56) to calculate the continuation functions $b(y, a)$. We first solve the equations for $b_{L}(\tau)$ and $b_{H}(\tau)$, and then for each $\tau$, we compute 
the corresponding payoff $a=V_{\tau}(y)$ for the agent. Figure 13 depicts the investor's payoff as a function of the agent's payoff in the low and high states for the parameters $r=0.05, \gamma=$ $0.1, \lambda=1, \alpha=0$. Note, that although the liquidation value of the project is zero in both states, $\lim _{a \rightarrow 0} b\left(y_{H}, a\right)>0$. This is because of the persistency of the cash flow process.

\subsection{The Implementation of the Optimal Continuous Time Contract}

In the discrete time setting, we have shown that the optimal contract can be implemented using an equity and a credit line with performance pricing. The investor who owns the fraction $(1-\lambda)$ of the equity opens the credit line to the agent who is the owner of the remaining shares of the firm. The credit line is characterized by the credit limit $C^{L}=V_{\infty}\left(y_{H}\right)-V_{1}\left(y_{H}\right)$ and the interest rate that increases with the balance. We now characterize the implementation of the optimal contract in the continuous time setting.

Theorem 6 The optimal contract in the continuous time setting can be implemented using a combination of equity and a credit line with performance pricing. The agent holds the fraction $\lambda$ of the equity, while the investor holds the remaining $(1-\lambda)$. The credit line has the credit limit

$$
\tilde{C}^{L}=\frac{1}{2 r}+\frac{1}{2(2 \gamma+r)}
$$

The earliest default time $\tau$ corresponds to the following balance on the credit line:

$$
M(\tau)=\frac{e^{-r \tau}}{2 r}+\frac{e^{-(2 \gamma+r) \tau}}{2(2 \gamma+r)}
$$

The interest rate charged on the balance $M(\tau)$ is given by

$$
\tilde{r}^{C}(M(\tau))=\frac{1+e^{-2 \gamma \tau}}{\frac{1}{r}+\frac{e^{-2 \gamma \tau}}{2 \gamma+r}} .
$$

Proof. The parameter $\lambda$ is not affected when we reduce the length $\Delta$ of a period. So, the optimal shares of the firm's equity held by the agent and the investor are the same as in discrete time.

The credit limit in the discrete time is equal to the first best value $V_{\infty}\left(y_{H}\right)$ of the project in the high state less the expected present value of the next period cash flows $V_{1}\left(y_{H}\right)$. As $\Delta \rightarrow 0, V_{1}\left(y_{H}\right) \rightarrow 0$, and $V_{\infty}\left(y_{H}\right) \rightarrow \tilde{V}_{\infty}(y) \equiv \frac{1}{2 r}+\frac{1}{2(2 \gamma+r)}$, which proves (66).

Theorem 2 in discrete time says that for the balance

$$
M(\tau)=V_{\infty}\left(y_{H}\right)-V_{\tau}\left(y_{H}\right),
$$




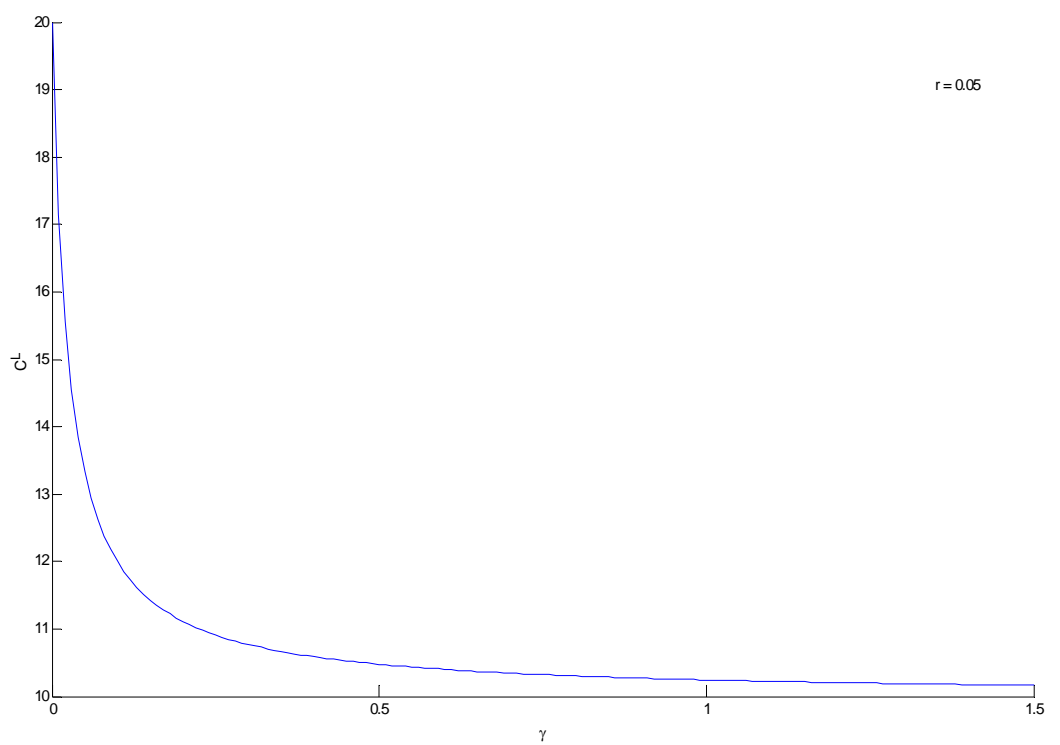

Figure 14: Creditl limit $\tilde{C}^{L}$ as a function of $\gamma$.

the interest rate on the credit line is given by

$$
{ }^{C}(M(\tau))=\frac{V_{\tau}\left(y_{H}\right)-V_{\tau-1}\left(y_{H}\right)}{M_{\tau}} .
$$

Replacing $V_{\infty}\left(y_{H}\right)$ and $V_{\tau}\left(y_{H}\right)$ with $\tilde{V}_{\infty}\left(y_{H}\right)$ and $\tilde{V}_{\tau}\left(y_{H}\right)$ yields $(67)$ and $(68)$.

One can verify that equation (68) is equivalent to

$$
\tilde{r}^{C}(M(\tau)) M(\tau)=\frac{V_{\tau}\left(y_{H}\right)}{\partial \tau} .
$$

The intuition behind (69) is as follows. If the agent makes the interest payment $\tilde{r}^{C}(M(\tau)) M(\tau)$, the balance on the credit line does not change. It buys the agent an extra time to default, which provides an opportunity to steal more cash flows in the future. The expected present value of the additional cash flows the agent can steal is equal to $\frac{V_{\tau}\left(y_{H}\right)}{\partial \tau}$. Equation (69) follows from the fact that under the optimal contract, the agent is indifferent between stealing and paying off the credit line.

Since the credit limit and interest rate are characterized by the smooth functions (66) (68), it makes it easier to analyze the properties of the credit line in continuous time. The credit limit $\tilde{C}^{L}$ decreases in both the risk-free interprets rate $r$ and the intensity $\gamma$. This is because the credit limit is equal to the first best value of the project in the high state. An increase in the interest rate leads to a lower expected present value of the future cash flows. An increase in $\gamma$ leads to higher chances of switching from the high to the low state, which 


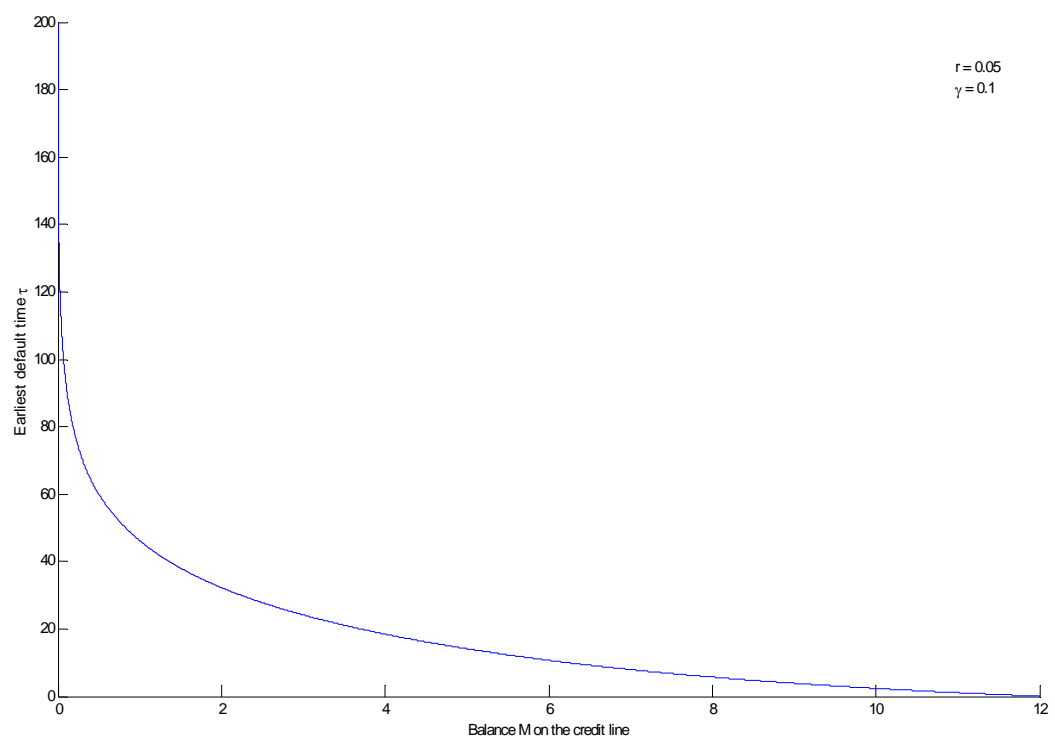

Figure 15: Earliest default time $\tau$ as a function of the balance $M$ on the credit line.

decreases the first best value of the project in the high state. For a given $r$, the smallest credit limit is equal to $\frac{1}{2 r}$, which is achieved when $\gamma \rightarrow \infty$. When $\gamma$ is high, the cash flow process switches between 0 and 1 so frequently that the average cash flow is 0.5 , no matter what the time zero state was. Hence, the first best value of the project is $\frac{1}{2 r}$. On the other hand, for a small $\gamma$, switching from 1 to 0 is highly unlikely. As $\gamma \rightarrow 0$, the credit limit reaches its highest value $\frac{1}{r}$. Thus, the highest possible credit limit is twice as high as the smallest one. Figure 14 shows how the credit limit changes with $\gamma$ when $r=0.05$.

Equation (67) relates the balance $M$ on the credit line to the earliest default time $\tau$. Given the current balance, the earliest default time $\tau$ corresponds to the default time in the case the agent always reports zero cash flows. Figure 15 plots $\tau$ as a function of $M$ for parameter $r=0.05$ and $\gamma=0.1$. Not surprisingly, the higher the balance the shorter the earliest default time is. Note that the earliest default time goes to infinity near zero balance. This is because the balance grows very slowly when it is low.

Now, let's consider the optimal interest rate on the credit line $\tilde{r}^{C}$ as a function of the earliest default time $\tau$. Equation (68) can be rewritten as follows:

$$
\tilde{r}^{C}(M(\tau))=r+\frac{2 r \gamma}{r+(2 \gamma+r) e^{2 \gamma \tau}}
$$

Equation (70) clearly shows that the interest on the credit line is greater than the riskfree interest rate and decreases with the earliest default time $\tau$. Since the balance $M(\tau)$ is a decreasing function of $\tau$, the interest rate $\tilde{r}^{C}(M(\tau))$ increases with the balance. As 


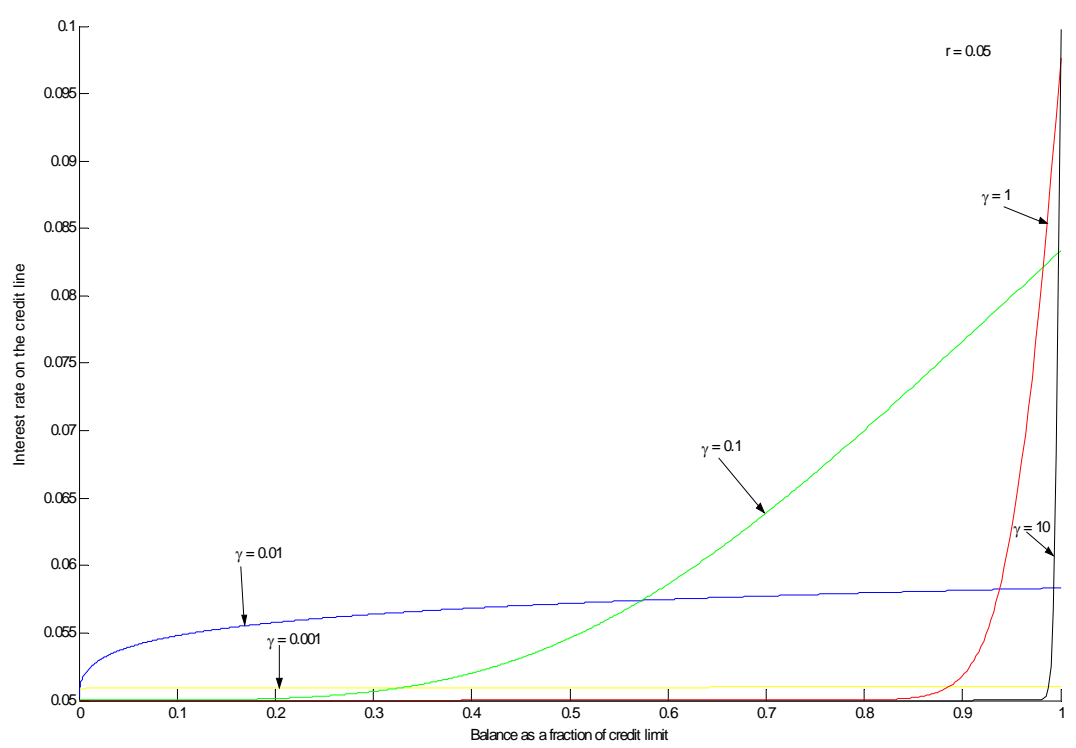

Figure 16: Optimal interest structure in continuous time.

$\tau \rightarrow \infty, M(\tau) \rightarrow 0, \tilde{r}^{C}(M(\tau)) \rightarrow r$. On the other hand, for $\tau=0, M(0)=C^{L}$, and $\tilde{r}^{C}\left(C^{L}\right)=r+\frac{r \gamma}{\gamma+r}$. Hence, for all $\tau \geq 0$

$$
r \leq \tilde{r}^{C}(M(\tau)) \leq r+\frac{r \gamma}{\gamma+r}
$$

The upper bound $r+\frac{r \gamma}{\gamma+r}$ increases with the intensity $\gamma$. However, for any $\gamma$ and $\tau$,

$$
r \leq \tilde{r}^{C}(M(\tau)) \leq 2 r
$$

Thus, our model predicts that performance pricing in the optimal contract does not lead to big changes in the interest rate.

While for small $\tau$, the interest rate $\tilde{r}^{C}$ increases with $\gamma$, we observe an opposite effect when $\tau$ is sufficiently large. Because of $e^{2 \gamma \tau}$ in the denominator in equation (70), the interest rate decreases with $\gamma$ for large $\tau$. Thus, a higher degree of correlation of the cash flows leads to higher interest rate at the beginning of the credit line and low interest rate at the end of the credit line. Figure 16 depicts the optimal interest rate $\tilde{r}^{C}$ as a function of $\frac{M}{C^{L}}$ for different values of the intensity $\gamma$. 


\section{Conclusion}

This paper presents a model of security design in a setting with an agency problem. An agent seeks external financing for a project that, if initiated, generates Markov cash flows whose realizations are unobservable and unverifiable by an outside investor. The agent has an ability to secretly divert the cash flows for his own consumption. The investor who cannot detect stealing has the right to liquidate the project. We characterize an optimal contract between the investor and the agent in this setting. We show that the optimal contract can be implemented using a credit line with an interest rate that increases with the outstanding balance on the credit line. The balance on the credit line can be considered as a performance measure that reflects all the relevant information regarding the past cash flow realizations.

In practice, a line of credit with performance pricing is the most prevalent form of banking lending. Our model demonstrates that a credit line with performance pricing is a part of the optimal contract in the setting with the agency problem. This finding is in line with the recent empirical studies by Asquith, Beatty and Weber (2002), Bradley and Roberts (2004), Dichev and Skinner (2004), which suggest that performance pricing, along with other debt covenants, is used to mitigate agency costs.

On the technical side, we develop a new recursive method to deal with correlated hidden states in dynamic settings. Unlike in the case with independent cash flows, the agent's preferences regarding continuation contracts are no longer the common knowledge in the setting with the correlated cash flows. A major challenge in reformulating the contracting problem recursively in this situation is to write incentive compatibility constraints. After reporting the low cash flow when the high cash flow is realized, the agent faces the same continuation contract that he would get after the low cash flow realized. However, the agent's continuation payoffs after the deviation is different from his continuation payoff after the low cash flow realization, due to the fact that the distribution of the future cash flows is determined by the current cash flow. A key element of our analysis is that we are able to find a one-to-one mapping between the agent's continuation payoffs in the states with the low and high cash flows. This allows us to formulate the contracting problem recursively using the agent's continuation payoff as a state variable and obtain a closed-form solution for the optimal contract. We also believe that this approach is not only applicable to our setting, but can also be used in other dynamic principal-agent models with correlated hidden states.

\section{Appendix}

\subsection{Properties of the Markov Cash Flows}

Lemma 5 Given $Q\left(y_{H}\right) \geq Q\left(y_{L}\right)$, conditional expectations of future cash flows satisfy the following inequalities. For any $k, s>0$, 
(i) $E\left[Y_{t+k} \mid Y_{t}=y_{H}\right] \geq E\left[Y_{t+k} \mid Y_{t}=y_{L}\right]$

(iia) $E\left[Y_{t+k} \mid Y_{t}=y_{H}\right] \geq E\left[Y_{t+k+s} \mid Y_{t}=y_{H}\right]$

(iib) $E\left[Y_{t+k} \mid Y_{t}=y_{L}\right] \leq E\left[Y_{t+k+s} \mid Y_{t}=y_{L}\right]$

(iii) $\frac{E\left[Y_{t+k} \mid Y_{t}=y_{H}\right]}{E\left[Y_{t+k+1} \mid Y_{t}=y_{H}\right]} \geq \frac{E\left[Y_{t+k+s} \mid Y_{t}=y_{H}\right]}{E\left[Y_{t+k+s+1} \mid Y_{t}=y_{H}\right]}$

(iv) $\lim _{k \rightarrow \infty} E\left[\left(Y_{t+k}-y_{L}\right) \mid Y_{t}=y_{H}\right]=\frac{Q\left(y_{L}\right)}{1-\left(Q\left(y_{H}\right)-Q\left(y_{L}\right)\right)}\left(y_{H}-y_{L}\right)$

Proof. (Lemma 5)

(i) Inequality (i) is proven by induction. For $k=1$, the inequality is true because $E\left[Y_{t+1} \mid Y_{t}\right]=y_{L}+Q\left(Y_{t}\right)\left(y_{H}-y_{L}\right)$ and $Q\left(y_{H}\right) \geq Q\left(y_{L}\right)$. Suppose that $E\left[Y_{t+k} \mid Y_{t}=y_{H}\right] \geq$ $E\left[Y_{t+k} \mid Y_{t}=y_{L}\right]$, which implies that $E\left[Y_{t+k+1} \mid Y_{t+1}=y_{H}\right] \geq E\left[Y_{t+k+1} \mid Y_{t+1}=y_{L}\right]$. Now, consider $E\left[Y_{t+k+1} \mid Y_{t}\right]$. By the law of iterated expectations

$$
\begin{aligned}
E\left[Y_{t+k+1} \mid Y_{t}\right] & =E\left[E\left[Y_{t+k+1} \mid Y_{t+1}\right] \mid Y_{t}\right] \\
& =Q\left(Y_{t}\right) E\left[Y_{t+k+1} \mid Y_{t+1}=y_{H}\right]+\left(1-Q\left(Y_{t}\right)\right) E\left[Y_{t+k+1} \mid Y_{t+1}=y_{L}\right] \\
& =E\left[Y_{t+k+1} \mid Y_{t+1}=y_{L}\right]+Q\left(Y_{t}\right)\left(E\left[Y_{t+k+1} \mid Y_{t+1}=y_{H}\right]-E\left[Y_{t+k+1} \mid Y_{t+1}=y_{L}\right]\right)
\end{aligned}
$$

Since $Q\left(y_{H}\right) \geq Q\left(y_{L}\right)$, it follows that $E\left[Y_{t+k+1} \mid Y_{t}=y_{H}\right] \geq E\left[Y_{t+k+1} \mid Y_{t}=y_{L}\right]$.

(ii) To show that (iia) holds, we use the law of iterated expectations again.

$$
\begin{aligned}
E\left[Y_{t+k+1} \mid Y_{t}=y_{H}\right] & =E\left[E\left[Y_{t+k+1} \mid Y_{t+1}\right] \mid Y_{t}=y_{H}\right] \\
& =Q\left(y_{H}\right) E\left[Y_{t+k+1} \mid Y_{t+1}=y_{H}\right]+\left(1-Q\left(y_{H}\right)\right) E\left[Y_{t+k+1} \mid Y_{t+1}=y_{L}\right] \\
& =Q\left(y_{H}\right) E\left[Y_{t+k} \mid Y_{t}=y_{H}\right]+\left(1-Q\left(y_{H}\right)\right) E\left[Y_{t+k} \mid Y_{t}=y_{L}\right] \\
& =E\left[Y_{t+k} \mid Y_{t}=y_{H}\right]-\left(1-Q\left(y_{H}\right)\right)\left(E\left[Y_{t+k} \mid Y_{t}=y_{H}\right]-E\left[Y_{t} \mid Y_{t}=y_{L}\right]\right) .
\end{aligned}
$$

Using (i), we see that $E\left[Y_{t+k} \mid Y_{t}=y_{H}\right] \geq E\left[Y_{t+k+1} \mid Y_{t}=y_{H}\right]$. Repeating this argument yields (iia) for any $s$. Part (iib) is proven the same way.

(iii) By the law of iterated expectations,

$$
\begin{aligned}
& E\left[Y_{t+k+s+1} \mid Y_{t}=y_{H}\right] E\left[Y_{t+k} \mid Y_{t}=y_{H}\right]-E\left[Y_{t+k+s} \mid Y_{t}=y_{H}\right] E\left[Y_{t+k+1} \mid Y_{t}=y_{H}\right] \\
= & \left(Q\left(y_{H}\right) E\left[Y_{t+k+s} \mid Y_{t}=y_{H}\right]+\left(1-Q\left(y_{H}\right)\right) E\left[Y_{t+k+s} \mid Y_{t}=y_{L}\right]\right) E\left[Y_{t+k} \mid Y_{t}=y_{H}\right] \\
& -E\left[Y_{t+k+s} \mid Y_{t}=y_{H}\right]\left(Q\left(y_{H}\right) E\left[Y_{t+k} \mid Y_{t}=y_{H}\right]+\left(1-Q\left(y_{H}\right)\right) E\left[Y_{t+k} \mid Y_{t}=y_{L}\right]\right) \\
= & \left(1-Q\left(y_{H}\right)\right)\left(E\left[Y_{t+k+s} \mid Y_{t}=y_{L}\right] E\left[Y_{t+k} \mid Y_{t}=y_{H}\right]-E\left[Y_{t+k+s} \mid Y_{t}=y_{H}\right] E\left[Y_{t+k} \mid Y_{t}=y_{L}\right]\right) \\
\geq & 0
\end{aligned}
$$

The last inequality follows from (iia) and (iib). 
(iv) The cash flow process has a unique stationary distribution. One can verify that $\lim _{k \rightarrow \infty} P\left(Y_{t+k}=y_{H} \mid Y_{t}=y_{H}\right)=\frac{Q\left(y_{L}\right)}{1-\left(Q\left(y_{H}\right)-Q\left(y_{L}\right)\right)}$.

\subsection{Proof of Lemma 1}

Let $\left\{\tilde{y}_{k}\left(y^{k} \mid y^{t-1}, \hat{y}^{t-1}\right)\right\}_{k=t}^{T}$ denote a continuation reporting strategy after a history $y^{t-1}$ of the realized cash flows, and a history $\hat{y}^{t-1}$ of the reported cash flows. We the contract $\sigma$ is incentive compatible after $\left(y^{t-1}, \hat{y}^{t-1}\right)$, provided the project is active at the beginning of period $t$, if for all $\left\{\tilde{y}_{k}\left(y^{k} \mid y^{t-1}, \hat{y}^{t-1}\right)\right\}_{k=t}^{T}$,

$$
\begin{aligned}
& E\left[\sum_{k=t}^{T} \beta^{k-t} \frac{P_{k}\left(\hat{y}^{t-1}, Y_{t}, y^{k-t}\right)}{P_{t}\left(\hat{y}^{t-1}, Y_{t}\right)} d_{t}\left(\hat{y}^{t-1}, Y_{t}, y^{k-t}\right) \mid Y_{t}\right] \\
\geq & E\left[\sum_{k=t}^{T} \beta^{k-t} \frac{P_{k}\left(\hat{y}^{t-1}, \tilde{y}_{k}\left(y^{k} \mid y^{t-1}, \hat{y}^{t-1}\right)\right)}{P_{t}\left(\hat{y}^{t-1}, Y_{t}\right)}\left(\begin{array}{c}
\lambda\left(Y_{k}-\tilde{y}_{k}\left(y^{k} \mid y^{t-1}, \hat{y}^{t-1}\right)\right) \\
+d_{t}\left(\hat{y}^{t-1}, \tilde{y}_{k}\left(y^{k} \mid y^{t-1}, \hat{y}^{t-1}\right)\right)
\end{array}\right) \mid Y_{t}\right] .
\end{aligned}
$$

First, we consider the case in which the agent has been telling the truth until time $t$. If the contract is not incentive compatible after $\left(y^{t-1}, y^{t-1}\right)$, then there is a continuation reporting strategy $\left\{\tilde{y}_{k}\left(y^{k} \mid y^{t-1}, y^{t-1}\right)\right\}_{k=t}^{T}$ that results in a higher continuation payoff than the truthtelling. However, the existence of $\left\{\tilde{y}_{k}\left(y^{k} \mid y^{t-1}, y^{t-1}\right)\right\}_{k=t}^{T}$ violates the time zero incentive compatibility constraint. A reporting strategy that consists of truth-telling until time $t$, truth telling after all histories other than $y^{t-1}$ after time $t$, and $\left\{\tilde{y}_{k}\left(y^{k} \mid y^{t-1}, y^{t-1}\right)\right\}_{k=t}^{T}$ after $y^{t-1}$ is an improvement on the truth-telling. This contradicts the assumption that the contract is incentive compatible.

Suppose the contract is not incentive compatible after $\left(y^{t-1}, \hat{y}^{t-1}\right)$, i.e. there exists a continuation reporting strategy $\left\{\bar{y}_{k}\left(y^{k} \mid y^{t-1}, \hat{y}^{t-1}\right)\right\}_{k=t}^{T}$ that delivers higher continuation payoff for the agent than truth-telling. The terms of the contracts after date $t-1$ are determined by the history $\hat{y}^{t-1}$ of reported cash flows, while the distribution of the future cash flows depends on the realization of the cash flow in period $t$. Continuation strategy $\bar{y}$ must bring an improvement on truth-telling either after $y_{t}=y_{L}$, or $y_{t}=y_{H}$, or in the both cases. Let's assume that the agent's continuation payoff is increased after $y_{t}=y_{L}$. To show that this violates the time zero incentive compatibility constraint, we consider the following deviation from the truth-telling strategy. Let strategy $y^{\prime}$ consist of truth-telling until time $t$ and after all histories of cash flows other than $\left(\hat{y}^{t-1}, y_{L}\right)$, and follows $\bar{y}$ after $\left(\hat{y}^{t-1}, y_{L}\right)$. Thus, the only difference between the truth-telling strategy and $y^{\prime}$ occurs after the cash flow history $\left(\hat{y}^{t-1}, y_{L}\right)$ is realized. Since $\left(\hat{y}^{t-1}, y_{L}\right)$ occurs with positive probability and $\bar{y}$ does better than the truth-telling after $\left(\hat{y}^{t-1}, y_{L}\right)$, strategy $y^{\prime}$ results in an improvement on truth-telling, violating the time zero incentive compatibility constraint. The same argument applies if the agent's continuation payoff is increased after $y_{t}=y_{H}$. 


\subsection{Proof of Theorem 1}

We first argue that if for some $t$ and $\left(y^{t-1}, y_{H}\right),(5)$ is violated, then the contract is not incentive compatible. Indeed, the strategy in which the agent lies only at date $t$, given $\left(y^{t-1}, y_{H}\right)$, will be an improvement on truth-telling, contradicting to the fact that (4) is satisfied.

Now, suppose that the contract $\sigma=(d, p)$ is such that (5) is always satisfied. To show that $\sigma$ is incentive compatible, consider an arbitrary reporting strategy $\tilde{y}$. Let $k \leq T$ be the last period when $\tilde{y}$ can recommend lying. Let $\hat{y}$ be a reporting strategy that coincides with $\tilde{y}$ before time $k$ and is truth-telling onward. Since both strategies $\tilde{y}$ and $\hat{y}$ result in the same income flows for the agent, as well as the same termination probabilities, before date $k$, and inequality (5) is satisfied at date $k$, then the date zero agent's expected payoff under the strategy $\hat{y}$ is equal to or greater than the corresponding payoff under the strategy $\tilde{y}$. Applying this argument inductively, we conclude $\tilde{y}$ cannot improve on truth-telling if (5) is satisfied. This proves that the contract is incentive compatible as long as (5) is satisfied for all time periods $t \leq T$, and all histories $y^{t}$.

\subsection{Proof of Lemma 2}

We use the definition of $V_{\tau}\left(Y_{t-1}\right)$ and the law of iterated expectations to prove the lemma.

$$
\begin{aligned}
V_{\tau+1}\left(Y_{t-1}\right) & =E\left[\sum_{k=1}^{n_{\tau}+1} \beta^{k}\left(Y_{t-1+k}-y_{L}\right)+l_{\tau} \beta^{\left(n_{\tau}+2\right)}\left(Y_{t+n_{\tau}+1}-y_{L}\right) \mid Y_{t-1}\right] \\
& =E\left[\beta\left(\left(Y_{t}-y_{L}\right)+\sum_{k=1}^{n_{\tau}} \beta^{k}\left(Y_{t+k}-y_{L}\right)+l_{\tau} \beta^{\left(n_{\tau}+1\right)}\left(Y_{t+n_{\tau}+1}-y_{L}\right)\right) \mid Y_{t-1}\right] \\
& =E\left[\beta\left(\left(Y_{t}-y_{L}\right)+E\left[\sum_{k=1}^{n_{\tau}} \beta^{k}\left(Y_{t+k}-y_{L}\right)+l_{\tau} \beta^{\left(n_{\tau}+1\right)}\left(Y_{t+n_{\tau}+1}-y_{L}\right) \mid Y_{t}\right]\right) \mid Y_{t-1}\right] \\
& =E\left[\beta\left(\left(Y_{t}-y_{L}\right)+V_{\tau}\left(Y_{t}\right)\right) \mid Y_{t-1}\right]
\end{aligned}
$$

\subsection{Proof of Propositions 2-5.}

We prove Propositions 2-5 by induction. Assuming that certain properties of the contract $\sigma^{*}$ hold in periods $t+1$ through $T$, we verify that those properties must hold in the period $t$.

\subsubsection{Proof of Proposition 2.}

Let $\theta_{t}\left(y_{t}\right)$ denote the slope of the line connecting the points of liquidation payoffs $\left(0, L_{t}\left(y_{t}\right)\right)$ and 
$\left(a^{L}\left(y_{t}\right), b_{t}^{e}\left(y_{t}, a^{L}\left(y_{t}\right)\right)\right)$. By the definition,

$$
\theta_{t}\left(y_{t}\right)=\frac{b_{t}^{e}\left(y_{t}, a^{L}\left(y_{t}\right)\right)-L_{t}\left(y_{t}\right)}{a^{L}\left(y_{t}\right)}
$$

To prove Proposition 2 we need the following Lemma.

Lemma 6 The slope $\theta_{t}\left(y_{t}\right)$ does not depend on $y_{t} \in\left\{y_{L}, y_{H}\right\}$ and is given by

$$
\theta_{t}\left(y_{t}\right)=\frac{1}{\lambda\left(y_{H}-y_{L}\right)}\left[(1-\alpha)\left(y_{H}-y_{L}\right)+\left(b_{t+1}^{d}\left(y_{H}, \lambda\left(y_{H}-y_{L}\right)\right)-L_{t+1}\left(y_{H}\right)\right)\right]
$$

Proof. (Lemma 6) If $a_{t}^{e}=a^{L}\left(y_{t}\right)$, then according to (31)-(33) the agent's continuation payoff in the next period after the cash flow realization will be $a_{t+1}^{d}\left(y_{t+1}\right)=\lambda\left(y_{t+1}-y_{L}\right)$. Using the fact that $b_{t+1}^{d}\left(y_{L}, 0\right)=L_{t+1}\left(y_{L}\right)$, we can express the investor's continuation payoff $b_{t}^{d}\left(y_{t}, a^{L}\left(y_{t}\right)\right)$ as follows:

$$
b_{t}^{d}\left(y_{t}, a^{L}\left(y_{t}\right)\right)=\beta\left[Q\left(y_{t}\right)\left(y_{H}+b_{t+1}^{d}\left(y_{H}, \lambda\left(y_{H}-y_{L}\right)\right)\right)+\left(1-Q\left(y_{t}\right)\right)\left(y_{L}+L_{t+1}\left(y_{L}\right)\right)\right] .
$$

Recall that

$$
a^{L}\left(y_{t}\right)=\beta Q\left(y_{t}\right) \lambda\left(y_{H}-y_{L}\right)
$$

and

$$
L_{t}\left(y_{t}\right)=\sum_{k=t+1}^{T} \beta^{k-t} y_{L}+\alpha V_{T-t}\left(y_{t}\right) .
$$

Using the Law of iterated expectations we can represent $L_{t}\left(y_{t}\right)$ as follows:

$$
L_{t}\left(y_{t}\right)=\beta\left[Q\left(y_{t}\right)\left(y_{L}+\alpha\left(y_{H}-y_{L}\right)+L_{t+1}\left(y_{H}\right)\right)+\left(1-Q\left(y_{t}\right)\right)\left(y_{L}+L_{t+1}\left(y_{L}\right)\right)\right] .
$$

Substituting (73), (74) and (75) into (71) we obtain formula (72).

Proof. (Proposition 2) The investor chooses the termination policy to maximize his continuation payoff, given the agent's continuation payoff. Since the agent and the investor get payoffs 0 and $L_{t}\left(y_{t}\right)$ in the case of termination, and the project can be terminated probabilistically, all payoffs are feasible within the convex hull of $\left(0, L_{t}\left(y_{t}\right)\right)$ and the end-of-period payoff possibility set, whose upper frontier is given by $b_{t}^{e}$. Let $\left(\tilde{a}_{t}^{L}\left(y_{t}\right), b_{t}^{e}\left(y_{t}, \tilde{a}_{t}^{L}\left(y_{t}\right)\right)\right)$ be the point, where the line originating from $\left(0, L_{t}\left(y_{t}\right)\right)$ is tangent to $b_{t}^{e}$. This line represents the highest possible continuation payoff for the investor before the liquidation decision has been made in period $t$, given the agent's continuation payoff belongs to the interval $\left(0, \tilde{a}_{t}^{L}\left(y_{t}\right)\right)$.

To prove the proposition we need to show that $\tilde{a}_{t}^{L}\left(y_{t}\right)=\lambda V_{1}\left(y_{t}\right)$. To do this we will show that the slope of the line connecting the liquidation payoff point $\left(0, L_{t}\left(y_{t}\right)\right)$ with the point 
$\left(\lambda V_{1}\left(y_{t}\right), b_{t}^{e}\left(y_{t}, \lambda V_{1}\left(y_{t}\right)\right)\right)$ is greater than the right-hand-side derivative of $b_{t}^{e}$ at $\lambda V_{1}\left(y_{t}\right)$. The slope of the line is given by (72). Consider the right-hand-side derivative of $b_{t}^{e}$, taken at $a_{t}^{L}\left(y_{t}\right) \equiv \lambda V_{1}\left(y_{t}\right)$. Using (31)-(33), we can write that for $a_{t}^{e}=\lambda V_{\tau}\left(y_{t}\right)$ with $\tau \geq 1$

$$
\begin{aligned}
b_{t}^{d}\left(y_{t}, a_{t}^{e}\right)= & \beta\left(1-Q\left(y_{t}\right)\right)\left(y_{L}+b_{t+1}^{d}\left(y_{L}, a_{t+1}^{d}\left(y_{L}\right)\right)\right) \\
& +\beta Q\left(y_{t}\right)\left(y_{H}+b_{t+1}^{d}\left(y_{H}, a_{t+1}^{d}\left(y_{H}\right)\right)\right),
\end{aligned}
$$

where

$$
a_{t+1}^{d}\left(y_{t+1}\right)=\lambda V_{\tau-1}\left(y_{t}\right)+\lambda\left(y_{t}-y_{L}\right) .
$$

Differentiating (76) with respect to $a_{t}^{e}$ gives

$$
\begin{aligned}
\frac{\partial b_{t}^{d}\left(y_{t}, a_{t}^{e}\right)}{\partial a_{t}^{e}}= & \nu_{t}\left(y_{t}, a_{t+1}^{d}\left(y_{L}\right)\right) \frac{\partial b_{t+1}^{d}\left(y_{L}, a_{t+1}^{d}\left(y_{L}\right)\right)}{\partial a_{t+1}^{d}\left(y_{L}\right)} \\
& +\nu_{t}\left(y_{t}, a_{t+1}^{d}\left(y_{H}\right)\right) \frac{\partial b_{t+1}^{d}\left(y_{H}, a_{t+1}^{d}\left(y_{H}\right)\right)}{\partial a_{t+1}^{d}\left(y_{H}\right)},
\end{aligned}
$$

where $\nu_{t}\left(y_{t}, a_{t+1}^{d}\left(y_{H}\right)\right)=\beta Q\left(y_{t}\right) \frac{\partial a_{t+1}^{d}\left(y_{H}\right)}{\partial a_{t}^{e}}$ and $\nu_{t}\left(y_{t}, a_{t+1}^{d}\left(y_{L}\right)\right)=\beta\left(1-Q\left(y_{t}\right)\right) \frac{\partial a_{t+1}^{d}\left(y_{L}\right)}{\partial a_{t}^{e}}$. Using (13), one can verify that

$$
\nu_{t}\left(y_{t}, a_{t+1}^{d}\left(y_{L}\right)\right)=\left(1-Q\left(y_{t}\right)\right) \frac{E\left[\left(Y_{t+n_{\tau}+1}-y_{L}\right) \mid Y_{t+1}=y_{L}\right]}{E\left[\left(Y_{t+n_{\tau}+1}-y_{L}\right) \mid Y_{t}=y_{t}\right]}
$$

and that

$$
\begin{aligned}
\nu_{t}\left(y_{t}, a_{t+1}^{d}\left(y_{H}\right)\right) & =Q\left(y_{t}\right) \frac{E\left[\left(Y_{t+n_{\tau}+1}-y_{L}\right) \mid Y_{t+1}=y_{H}\right]}{E\left[\left(Y_{t+n_{\tau}+1}-y_{L}\right) \mid Y_{t}=y_{t}\right]} \\
& =1-\nu_{t}\left(y_{t}, a_{t+1}^{d}\left(y_{L}\right)\right) .
\end{aligned}
$$

Thus, the right-hand-side derivative of $b_{t}^{e}$ at the point $\lambda V_{1}\left(y_{t}\right)$ can be written as

$$
\frac{\partial b_{t}^{d}\left(y_{t}, \lambda V_{1}\left(y_{t}\right)+\right)}{\partial a_{t}^{d}}=\frac{\partial b_{t+1}^{d}\left(y_{L}, 0+\right)}{\partial a_{t+1}^{d}\left(y_{L}\right)} \nu\left(y_{t}, 0+\right)+\frac{\partial b_{t+1}^{d}\left(y_{H}, \lambda\left(y_{H}-y_{L}\right)+\right)}{\partial a_{t+1}^{d}\left(y_{H}\right)}\left(1-\nu\left(y_{t}, 0+\right)\right)
$$

where

$$
\nu\left(y_{t}, 0+\right)=\left(1-Q\left(y_{t}\right)\right) \frac{E\left[Y_{t+2} \mid Y_{t+1}=y_{L}\right]}{E\left[Y_{t+2} \mid Y_{t}=y_{t}\right]},
$$

and "+" indicates the fact that we are considering the right neighborhood of a given point. 
Subtracting (81) from (72) and rearranging terms gives

$$
\begin{aligned}
& \theta\left(y_{t}\right)-\frac{\partial b_{t}^{d}\left(y_{t}, \lambda V_{1}\left(y_{t}\right)+\right)}{\partial a_{t}^{d}} \\
= & {\left[\frac{(1-\alpha)}{\lambda}-\frac{\partial b_{t+1}^{d}\left(y_{L}, 0+\right)}{\partial a_{t+1}^{d}\left(y_{L}\right)} \nu\left(y_{t}, 0+\right)\right] } \\
& +\left[\frac{b_{t+1}^{d}\left(y_{H}, \lambda\left(y_{H}-y_{L}\right)\right)}{\lambda\left(y_{H}-y_{L}\right)}-\frac{\partial b_{t+1}^{d}\left(y_{H}, \lambda\left(y_{H}-y_{L}\right)+\right)}{\partial a_{t+1}^{d}\left(y_{H}\right)}\left(1-\nu\left(y_{t}, 0+\right)\right)-\frac{L_{t+1}\left(y_{H}\right)}{\lambda\left(y_{H}-y_{L}\right)}\right] \\
\geq & {\left[\frac{(1-\alpha)}{\lambda}-\frac{\partial b_{t+1}^{d}\left(y_{L}, 0+\right)}{\partial a_{t+1}^{d}\left(y_{L}\right)} \nu\left(y_{t}, 0+\right)\right] } \\
& +\left[\frac{b_{t+1}^{d}\left(y_{H}, \lambda\left(y_{H}-y_{L}\right) \nu\left(y_{t}, 0+\right)\right)}{\lambda\left(y_{H}-y_{L}\right)}-\frac{L_{t+1}\left(y_{H}\right)}{\lambda\left(y_{H}-y_{L}\right)}\right] .
\end{aligned}
$$

The inequality follows from concavity of the function $b_{t+1}^{d}$.

By the assumption of our inductive argument, Proposition 2 and Lemma 6 hold in period $t+1$. Therefore, $b_{t+1}^{d}$ is affine on the interval $\left[0, \lambda V_{1}\left(y_{t}\right)\right]$, with $b_{t+1}^{d}\left(y_{t+1}, 0\right)=L_{t+1}\left(y_{t+1}\right)$, and its derivative on this interval is equal to the slope $\theta_{t+1}\left(y_{t+1}\right)$, which does not depend on $y_{t+1}$. Hence,

$$
\begin{aligned}
\frac{\partial b_{t+1}^{d}\left(y_{L}, 0+\right)}{\partial a_{t+1}^{d}} \nu\left(y_{t}, 0+\right) & =\theta_{t+1}\left(y_{L}\right) \nu\left(y_{t}, 0+\right) \\
& =\theta_{t+1}\left(y_{H}\right) \nu\left(y_{t}, 0+\right) \\
& =\frac{\partial b_{t+1}^{d}\left(y_{H}, 0+\right)}{\partial a_{t+1}^{d}} \nu\left(y_{t}, 0+\right)
\end{aligned}
$$

On the other hand,

$$
\frac{b_{t+1}^{d}\left(y_{H}, \lambda\left(y_{H}-y_{L}\right) \nu\left(y_{t}, 0+\right)\right)}{\lambda\left(y_{H}-y_{L}\right)}-\frac{L_{t+1}\left(y_{H}\right)}{\lambda\left(y_{H}-y_{L}\right)}=\frac{\partial b_{t+1}^{d}\left(y_{H}, 0+\right)}{\partial a_{t+1}^{d}} \nu\left(y_{t}, 0+\right)
$$

Substituting (83) and (84) into (82) gives

$$
\theta\left(y_{t}\right)-\frac{\partial b_{t}^{d}\left(y_{t}, \lambda V_{1}\left(y_{t}\right)+\right)}{\partial a_{t}^{d}} \geq \cdot \frac{(1-\alpha)}{\lambda} \geq 0
$$

This proves that the tangent point is equal to $a_{t}^{L}\left(y_{t}\right)=\lambda V_{1}\left(y_{t}\right)$, which gives us the desired result. 


\subsubsection{Proof of Proposition 3}

For $y \in\left\{y_{L}, y_{H}\right\}$, let $\frac{\partial b_{t}^{d}\left(y, a_{t}^{d}\right)}{\partial a_{t}^{d}}$ denote the right-hand-side derivative of the continuation function $b_{t}^{d}$. The proof of Proposition 3 follows from the following lemma.

Lemma 7 The continuation functions $b_{t}^{d}\left(Y_{t}, a_{t}^{d}\right)$ are concave in the agent's continuation payoff $a_{t}^{d}$. Moreover,

$$
\frac{\partial b_{t}^{d}\left(y_{L}, V_{\tau}\left(y_{L}\right)\right)}{\partial a_{t}^{d}} \geq \frac{\partial b_{t}^{d}\left(y_{H}, V_{\tau}\left(y_{H}\right)\right)}{\partial a_{t}^{d}}
$$

Proof. (Lemma 7). Since $b_{T}^{d}\left(y_{T}, a_{T}^{d}\right)=-a_{T}^{d}$, the statement of the lemma is true in the final period. We assume that the statement of the Lemma is true in period $t+1$. If $\tau \leq 1$, then $\frac{\partial b_{t}^{d}\left(y_{L}, V_{\tau}\left(y_{L}\right)\right)}{\partial a_{t}^{d}}=\frac{\partial b_{t}^{d}\left(y_{H}, V_{\tau}\left(y_{H}\right)\right)}{\partial a_{t}^{d}}$, according to Lemma 6 .

Let $\tau>1$. Using (78)-(80), we can represent a derivative the continuation function in period $t$ as the weighted average of derivatives of the continuation function in period $t+1$ :

$$
\begin{aligned}
\frac{\partial b_{t}^{d}\left(y_{t}, V_{\tau}\left(y_{t}\right)\right)}{\partial a_{t}^{d}}= & \nu\left(y_{t}, \tau\right) \frac{\partial b_{t+1}^{d}\left(y_{L}, \lambda V_{\tau-1}\left(y_{L}\right)\right)}{\partial a_{t+1}^{d}\left(y_{L}\right)} \\
& +\left(1-\nu\left(y_{t}, \tau\right)\right) \frac{\partial b_{t+1}^{d}\left(y_{H}, \lambda V_{\tau-1}\left(y_{H}\right)+\lambda\left(y_{H}-y_{L}\right)\right)}{\partial a_{t+1}^{d}\left(y_{H}\right)}
\end{aligned}
$$

where

$$
\nu_{t}\left(y_{t}, \tau\right)=\left(1-Q\left(y_{t}\right)\right) \frac{E\left[\left(Y_{t+n_{\tau}+1}-y_{L}\right) \mid Y_{t+1}=y_{L}\right]}{E\left[\left(Y_{t+n_{\tau}+1}-y_{L}\right) \mid Y_{t}=y_{t}\right]}
$$

Since by the assumption of induction, the statement of the lemma is true in period $t+1$,

$$
\begin{aligned}
\frac{\partial b_{t+1}^{d}\left(y_{L}, \lambda V_{\tau-1}\left(y_{L}\right)\right)}{\partial a_{t+1}^{d}\left(y_{L}\right)} & \geq \frac{\partial b_{t+1}^{d}\left(y_{H}, \lambda V_{\tau-1}\left(y_{H}\right)\right)}{\partial a_{t+1}^{d}\left(y_{H}\right)} \\
& \geq \frac{\partial b_{t+1}^{d}\left(y_{H}, \lambda V_{\tau-1}\left(y_{H}\right)+\lambda\left(y_{H}-y_{L}\right)\right)}{\partial a_{t+1}^{d}\left(y_{H}\right)},
\end{aligned}
$$

where the second inequality follows from the fact that $b_{t+1}^{d}\left(y_{H}, a_{t}^{d}\right)$ is concave in $a_{t}^{d}$.

Thus, to prove (85) it is enough to show that $\nu_{t}\left(y_{L}, \tau\right)>\nu_{t}\left(y_{H}, \tau\right)$. However, since $E\left[\left(Y_{t+n_{\tau}+1}-y_{L}\right) \mid Y_{t}=y_{L}\right]<E\left[\left(Y_{t+n_{\tau}+1}-y_{L}\right) \mid Y_{t}=y_{H}\right]$, it immediately follows that

$$
\nu_{t}\left(y_{L}, \tau\right)>\nu_{t}\left(y_{H}, \tau\right)
$$

which proves that the inequality (85) holds in period $t$.

Now it is easy to prove Proposition 3.

Proof. (Proposition 3). Consider Problem (22)-(25), in which the objective function is maximized by the pair of continuation payoffs $a_{t}^{d}\left(y_{L}\right)$ and $a_{t}^{d}\left(y_{H}\right)$ subject to the (IC), (PK), 
and (IR) constraints. Let the difference between them be denoted by $\xi \equiv a_{t}^{d}\left(y_{H}\right)-a_{t}^{d}\left(y_{L}\right)$. We will show that the objective function decreases in the difference $\xi$. Since the continuation payoffs satisfy the promise keeping constraint (PK) it must be the case that

$$
\begin{aligned}
a_{t}^{d}\left(y_{L}, \xi\right) & =a_{t}^{y}-Q\left(y_{t-1}\right) \xi \\
a_{t}^{d}\left(y_{H}, \xi\right) & =a_{t}^{y}+\left(1-Q\left(y_{t-1}\right)\right) \xi
\end{aligned}
$$

The investor's continuation payoff as a function of $\xi$ is given by

$$
\tilde{b}(\xi)=\left(1-Q\left(y_{t-1}\right)\right)\left(y_{L}+b_{t}^{d}\left(y_{L}, a_{t}^{d}\left(y_{L}, \xi\right)\right)+Q\left(y_{t-1}\right)\left(y_{H}+b_{t}^{d}\left(y_{H}, a_{t}^{d}\left(y_{H}, \xi\right)\right)\right) .\right.
$$

Taking the derivative of $\tilde{b}$ with respect to $\xi$, we obtain that

$$
\tilde{b}^{\prime}(\xi)=-Q\left(y_{t-1}\right)\left(1-Q\left(y_{t-1}\right)\right)\left(\frac{\partial b_{t}^{d}\left(y_{L}, a_{t}^{d}\left(y_{L}, \xi\right)\right)}{\partial a_{t}^{d}}-\frac{\partial b_{t}^{d}\left(y_{H}, a_{t}^{d}\left(y_{H}, \xi\right)\right)}{\partial a_{t}^{d}}\right) \leq 0,
$$

where the inequality follows from Lemma 7.

Since $\tilde{b}^{\prime}(\xi) \leq 0$, the solution of Problem (22)-(25) is obtained by choosing the smallest $\xi$ such that the (IC) constraint is satisfied, which corresponds to the case in which the (IC) constraint is satisfied with equality.

\subsubsection{Proof of Proposition 4}

Suppose the continuation contract $\sigma_{t}^{*}\left(y_{L}\right)$ results in the continuation payoff $a_{t}^{d}\left(y_{L}\right)$ for the agent after the low cash flow realization in period $t$. Lemma 1 says that since the contract $\sigma^{*}$ is incentive compatible, it is always optimal for the agent to report cash flows truthfully in the future, even if the agent deviated in the past. Given the probability of liquidation $p_{t}$ in period $t$, we can represent this payoff as the expectation of the agent's continuation payoffs in the next period:

$$
a_{t}^{d}\left(y_{L}\right)=E\left[\beta\left(1-p_{t}\right) a_{t+1}^{d}\left(Y_{t+1}\right) \mid Y_{t}=y_{L}\right],
$$

where $a_{t+1}^{d}\left(Y_{t+1}\right)$ denotes the continuation payoff for the agent in period $t+1$ under the continuation contract $\sigma_{t}^{*}\left(y_{L}\right)$ conditional on the cash flow $Y_{t+1}$.

Similarly, if the agent reports the low cash flow in period $t$, when, in fact, the high cash flow realized, the same contract results in the following continuation payoff after the deviation:

$$
c_{t}\left(a_{t}^{d}\left(y_{L}\right)\right)=E\left[\beta\left(1-p_{t}\right) a_{t+1}^{d}\left(Y_{t+1}\right) \mid Y_{t}=y_{H}\right] .
$$

In the equations (88), the expectation is taken over the same continuation payoffs $a_{t+1}^{d}\left(Y_{t+1}\right)$, but with respect to a different cash flow realization in period $t$.

We consider the cases with $a_{t}^{d}\left(y_{L}\right)<a^{L}\left(y_{L}\right)$ and $a_{t}^{d}\left(y_{L}\right)>a^{L}\left(y_{L}\right)$. 
Case 1: $a_{t}^{d}\left(y_{L}\right)>a^{L}\left(y_{L}\right)$. Let

$$
a_{t}^{d}\left(y_{L}\right)=\lambda V_{\tau}\left(y_{L}\right)
$$

for some $\tau \geq 1$. According to Proposition 2 and equations (31)-(34), the probability of liquidation $p_{t}$ is zero, and the continuation payoffs in the next period are given by

$$
a_{t+1}^{d}\left(Y_{t+1}\right)=\lambda V_{\tau-1}\left(Y_{t+1}\right)+\lambda\left(Y_{t+1}-y_{L}\right)
$$

Substituting this into (88) gives

$$
\begin{aligned}
c_{t}\left(a_{t}^{d}\left(y_{L}\right)\right) & =E\left[\beta\left(\lambda V_{\tau-1}\left(Y_{t+1}\right)+\lambda\left(Y_{t+1}-y_{L}\right)\right) \mid Y_{t}=y_{H}\right] \\
& =\lambda V_{\tau}\left(y_{H}\right),
\end{aligned}
$$

where the second equality follows from Lemma 2. Thus,

$$
c_{t}\left(\lambda V_{\tau}\left(y_{L}\right)\right)=\lambda V_{\tau}\left(y_{H}\right)
$$

Case 2: $a_{t}^{d}\left(y_{L}\right) \leq a^{L}\left(y_{L}\right)$. Again, let

$$
a_{t}^{d}\left(y_{L}\right)=\lambda V_{\tau}\left(y_{L}\right)
$$

for some $\tau<1$. Since $\tau<1, V_{\tau}(y)=\tau E\left[\beta\left(Y_{t+1}-y_{L}\right) \mid Y_{t}=y\right]$. Then, the probability of liquidation is given by $p_{t}=1-\tau$. If the project is not liquidated in period $t$, the next period continuation payoffs are given by

$$
a_{t+1}^{d}\left(Y_{t+1}\right)=\lambda\left(Y_{t+1}-y_{L}\right)
$$

in accordance with Proposition 2 and equations (31)-(34). Then, the equation (89) gives

$$
\begin{aligned}
c_{t}\left(a_{t}^{d}\left(y_{L}\right)\right) & =E\left[\beta \tau \lambda\left(Y_{t+1}-y_{L}\right) \mid Y_{t}=y_{H}\right] \\
& =\lambda V_{\tau}\left(y_{H}\right) .
\end{aligned}
$$

Thus, we proved Proposition 4 for Case 2 .

\subsubsection{Proof of Proposition 5}

According to Lemma 2,

$$
\frac{1}{\beta} V_{\tau+1}\left(Y_{t-1}\right)=E\left[V_{\tau}\left(Y_{t}\right)+\left(Y_{t}-y_{L}\right) \mid Y_{t-1}\right]
$$

Hence, the (PK) constraint is satisfied. 
According to $(30), a_{t}^{d}\left(y_{L}\right)=\lambda V_{\tau}\left(y_{L}\right)$ and $a_{t}^{d}\left(y_{H}\right)=\lambda V_{\tau}\left(y_{H}\right)+\lambda\left(y_{H}-y_{L}\right)$. Proposition 4 says that $c_{t}\left(a_{t}^{d}\left(y_{L}\right)\right)=\lambda V_{\tau}\left(y_{H}\right)$. Putting all together:

$$
a_{t}^{d}\left(y_{H}\right)=c_{t}\left(a_{t}^{d}\left(y_{L}\right)\right)+\lambda\left(y_{H}-y_{L}\right) .
$$

Thus, $a_{t}^{d}\left(y_{L}\right)$ and $a_{t}^{d}\left(y_{H}\right)$ satisfy the (IC) constraint as equality.

Substituting (30) into (22) gives $b_{t}^{y}\left(Y_{t-1}, \frac{1}{\beta} \lambda V_{\tau+1}\left(Y_{t-1}\right)\right)$.

\subsection{Proof of Proposition 7}

Proof. In order to prove that $\sigma^{*}$ is the optimal contract, we will demonstrate that for each date $t$ and for any agent's continuation payoff $a_{t}^{y}$, there is no continuation contract that results in the investor's payoff greater than $b_{t}^{y}\left(y_{t-1}, a_{t}^{y}\right)$.

Let $\tilde{\sigma}$ be an arbitrary incentive compatible contract, with the continuation payoffs after the cash flow realization in period $t$ for the agent and the investor given by $\tilde{a}_{t}^{d}\left(y_{t}\right)$, and $\tilde{b}_{t}^{d}\left(y_{t}, \tilde{a}_{t}^{d}\left(y_{t}\right)\right)$. The continuation payoff for the investor at the beginning of period $t$ is given by

$$
\tilde{b}_{t}^{y}\left(Y_{t-1}, a_{t}^{y}\right)=E_{t}\left[Y_{t}+\tilde{b}_{t}^{d}\left(Y_{t}, \tilde{a}_{t}^{d}\left(Y_{t}\right)\right) \mid Y_{t-1}\right] .
$$

Let $\tilde{c}_{t}$ denote a deviation payoff function, associated with the contract $\tilde{\sigma}$. Since, by assumption, $\tilde{\sigma}$ is incentive compatible, the agent's continuation payoffs $\tilde{a}_{t}^{d}$, associated with $\tilde{\sigma}$, must satisfy

$$
\tilde{a}_{t}^{d}\left(y_{H}\right) \geq \tilde{c}_{t}\left(\tilde{a}_{t}^{d}\left(y_{L}\right)\right)+\lambda\left(y_{H}-y_{L}\right) \text { for all } t \leq T \text {. }
$$

In the last period, $\tilde{b}_{T}^{d}\left(Y_{T}, \tilde{a}_{T}^{d}\left(Y_{T}\right)\right) \leq b_{T}^{d}\left(Y_{T}, \tilde{a}_{T}^{d}\left(Y_{T}\right)\right)$. We assume by induction that $\tilde{b}_{t}^{d}\left(Y_{t}, \tilde{a}_{t}^{d}\left(Y_{t}\right)\right) \leq b_{t}^{d}\left(Y_{t}, \tilde{a}_{t}^{d}\left(Y_{t}\right)\right)$.

In the proof of Proposition 3, we demonstrate that the function $E_{t}\left[Y_{t}+b_{t}^{d}\left(Y_{t}, \tilde{a}_{t}^{d}\left(Y_{t}\right)\right) \mid Y_{t-1}\right]$ (see equation (86)) increases as the difference $\tilde{a}_{t}^{d}\left(y_{H}\right)-\tilde{a}_{t}^{d}\left(y_{L}\right)$ decreases. Since $\tilde{b}_{t}^{d}\left(Y_{t}, \tilde{a}_{t}^{d}\left(Y_{t}\right)\right) \leq$ $b_{t}^{d}\left(Y_{t}, \tilde{a}_{t}^{d}\left(Y_{t}\right)\right)$, the only way that $\tilde{b}_{t}^{y}\left(Y_{t-1}, a_{t}^{y}\right)$ can be greater than $b_{t}^{y}\left(Y_{t-1}, a_{t}^{y}\right)$ is when $\tilde{a}_{t}^{d}\left(y_{H}\right)-\tilde{a}_{t}^{d}\left(y_{L}\right)<a_{t}^{d}\left(y_{H}\right)-a_{t}^{d}\left(y_{L}\right)$, where $a_{t}^{d}\left(y_{H}\right)$ and $a_{t}^{d}\left(y_{L}\right)$ the incentive compatibility constraint under the contract $\sigma^{*}$ :

$$
a_{t}^{d}\left(y_{H}\right)=c_{t}\left(a_{t}^{d}\left(y_{L}\right)\right)+\lambda\left(y_{H}-y_{L}\right)
$$

To illustrate that this is impossible that $\tilde{a}_{t}^{d}\left(y_{H}\right)-\tilde{a}_{t}^{d}\left(y_{L}\right)<a_{t}^{d}\left(y_{H}\right)-a_{t}^{d}\left(y_{L}\right)$, we will show that $\tilde{c}_{t}(a) \geq c_{t}(a)$. We will show recursively that equation (90) implies that $\tilde{c}_{t}(a) \geq c_{t}(a)$, for all $a \geq 0$, and $t \leq T$. This must be true in the last period $T$ for the contract $\tilde{\sigma}$ to be incentive compatible.

Suppose at time $t$,

$$
\tilde{c}_{t}(a) \geq c_{t}(a), \text { for all } a \geq 0
$$


Equations (90) and (91) imply that if $\tilde{a}_{t}^{d}\left(y_{L}\right)=\lambda V_{\tau}\left(y_{L}\right)$, then $\tilde{a}_{t}^{d}\left(y_{H}\right)=\lambda V_{\tau}\left(y_{H}\right)+$ $\lambda\left(y_{H}-y_{L}\right)+\varphi$, with $\varphi \geq 0$, and $\varphi>0$ if equation (91) holds with strict inequality.

Now, consider continuation payoffs in period $t-1$. Given the continuation payoffs $\tilde{a}_{t}^{d}\left(y_{L}\right)$ and $\tilde{a}_{t}^{d}\left(y_{H}\right)$ in period $t$, we can compute the continuation payoff in period $t-1$ :

$$
\begin{aligned}
\tilde{a}_{t-1}^{d}\left(y_{L}\right) & =E\left[\beta \tilde{a}_{t}^{d}\left(Y_{t}\right) \mid Y_{t-1}=y_{L}\right] \\
& =V_{\tau+1}\left(y_{L}\right)+Q\left(y_{L}\right) \beta \varphi \\
& =V_{\tau_{1}}\left(y_{L}\right),
\end{aligned}
$$

The deviation payoff $\tilde{c}_{t-1}\left(\tilde{a}_{t-1}^{d}\left(y_{L}\right)\right)$ in period $t-1$ can be obtained as follows:

$$
\begin{aligned}
\tilde{c}_{t-1}\left(\tilde{a}_{t-1}^{d}\left(y_{L}\right)\right) & =E\left[\beta \tilde{a}_{t}^{d}\left(Y_{t}\right) \mid Y_{t-1}=y_{H}\right] \\
& =V_{\tau+1}\left(y_{H}\right)+Q\left(y_{H}\right) \beta \varphi \\
& =V_{\tau_{2}}\left(y_{H}\right)
\end{aligned}
$$

for where parameters $\tau_{1}$ and $\tau_{2}$ are chosen so that the corresponding equalities in (93) and (92) hold.

In particular, $\tau_{1}$ solves

$$
\begin{aligned}
Q\left(y_{L}\right) \beta \varphi= & \left(1-l_{\tau}\right) \beta^{n_{\tau}+1} E\left[\left(Y_{n_{\tau}+1}-y_{L}\right) \mid Y_{0}=y_{L}\right] \\
& +\sum_{k=\tau+2}^{n_{\tau_{1}}} \beta^{k} E\left[\left(Y_{k}-y_{L}\right) \mid Y_{0}=y_{L}\right]+l_{\tau_{1}} \beta^{n_{\tau_{1}}+1} E\left[\left(Y_{n_{\tau_{1}}+1}-y_{L}\right) \mid Y_{0}=y_{L}\right],
\end{aligned}
$$

where $n_{\tau}$ is the biggest integer, such that $n_{\tau} \leq \tau$, and $l_{\tau}=\tau-n_{\tau}$. Similarly, $\tau_{2}$ solves

$$
\begin{aligned}
Q\left(y_{H}\right) \beta \varphi= & \left(1-l_{\tau}\right) \beta^{n_{\tau}+1} E\left[\left(Y_{n_{\tau}+1}-y_{L}\right) \mid Y_{0}=y_{H}\right] \\
& +\sum_{k=\tau+2}^{n_{\tau_{2}}} \beta^{k} E\left[\left(Y_{k}-y_{L}\right) \mid Y_{0}=y_{H}\right]+l_{\tau_{2}} \beta^{n_{\tau_{2}}+1} E\left[\left(Y_{n_{\tau_{2}}+1}-y_{L}\right) \mid Y_{0}=y_{H}\right],
\end{aligned}
$$

Using the fact that $E\left[\left(Y_{1}-y_{L}\right) \mid Y_{0}=y\right]=Q(y)\left(y_{H}-y_{L}\right)$ and Lemma 5 (iia) and (iib),

$$
\frac{E\left[\left(Y_{k}-y_{L}\right) \mid Y_{0}=y_{L}\right]}{Q\left(y_{L}\right) \beta \varphi} \geq \frac{E\left[\left(Y_{k}-y_{L}\right) \mid Y_{0}=y_{H}\right]}{Q\left(y_{H}\right) \beta \varphi}
$$

Therefore, $\tau_{1} \leq \tau_{2}$.

Recall that $c_{t}\left(V_{\tau_{1}}\left(y_{L}\right)\right)=V_{\tau_{1}}\left(y_{H}\right)$. The fact that $\tau_{1} \leq \tau_{2}$ gives us the desired result: $\tilde{c}_{t}\left(\tilde{a}_{t-1}^{d}\left(y_{L}\right)\right) \geq c_{t}\left(\tilde{a}_{t-1}^{d}\left(y_{L}\right)\right)$.

The function $\tilde{c}_{t}$ determines the difference between $\tilde{a}_{t}^{d}\left(y_{H}\right)$ and $\tilde{a}_{t}^{d}\left(y_{L}\right)$. The greater the value of the function $\tilde{c}_{t}$, the greater this difference is. Hence, $\tilde{b}_{t}^{y}\left(Y_{t-1}, a_{t}^{y}\right)$ cannot be greater than $b_{t}^{y}\left(Y_{t-1}, a_{t}^{y}\right)$. Consequently, $\tilde{\sigma}$ cannot be better than $\sigma^{*}$. 


\subsection{Proof of Proposition 8}

The only parameter affected by the uncertainty about the initial state $Y_{0}$ is the distribution of date 1 earning $Y_{1}$. The probability of $Y_{1}=y_{H}$ is given by $q_{1} \equiv Q\left(y_{H}\right) q_{H}+Q\left(y_{L}\right)\left(1-q_{H}\right)$. Conditional on the realization of $Y_{1}$, the agent's and the investor's continuation payoffs are given by $a_{1}^{d}\left(Y_{1}\right)$ and $b_{1}^{d}\left(Y_{1}, a_{1}^{d}\left(Y_{1}\right)\right)$.

Given the agent's payoff $a_{0}$, the investor chooses the agent's continuation payoffs $a_{1}^{d}(\cdot)$ to solve the following optimization problem:

$$
\begin{aligned}
b_{0}\left(a_{0}\right) & \equiv \max _{a_{1}^{d}(\cdot)} \beta E\left[Y_{1}+b_{1}^{d}\left(Y_{1}, a_{1}^{d}\left(Y_{1}\right)\right)\right] \\
\text { s.t. } a_{1}^{d}\left(y_{H}\right) & \geq c_{1}\left(a_{1}^{d}\left(y_{L}\right)\right)+\lambda\left(y_{H}-y_{L}\right) \\
a_{0} & =\beta E\left[a_{1}^{d}\left(Y_{1}\right)\right] \quad\left(P K_{1}\right)
\end{aligned}
$$

Our previous analysis of a similar problem indicates that $\left(\mathrm{IC}_{1}\right)$ binds. Letting $a_{0}^{e}\left(Y_{0}\right) \equiv$ $\beta E\left[a_{1}^{d}\left(Y_{1}\right) \mid Y_{0}\right]$, and $b_{0}^{e}\left(Y_{0}, a_{0}^{e}\left(Y_{0}\right)\right) \equiv \beta E\left[Y_{1}+b_{1}^{d}\left(Y_{1}, a_{1}^{d}\left(Y_{1}\right)\right) \mid Y_{0}\right]$ gives the result.

\subsection{Proof of Proposition 9}

The proof is by showing that $b_{0}^{\prime}\left(a_{0}\right) \geq-1$. Using the fact that $a_{0}=E\left[a_{0}^{e}\left(Y_{0}\right)\right], b_{0}\left(a_{0}\right)=$ $E\left[b_{0}^{e}\left(Y_{0}, a_{0}^{e}\left(Y_{0}\right)\right)\right]$ and $a_{0}^{e}\left(y_{H}\right)=c_{0}\left(a_{0}^{e}\left(y_{L}\right)\right)$, we can write

$$
b_{0}^{\prime}\left(a_{0}\right)=\frac{\frac{d b_{0}^{e}}{d a_{0}^{e}\left(y_{L}\right)}}{\frac{a_{0}}{d a_{0}^{e}\left(y_{L}\right)}}=\frac{\left(1-q_{H}\right) b_{0}^{e \prime}\left(y_{L}, a_{0}^{e}\left(y_{L}\right)\right)+q_{H} b_{0}^{e \prime}\left(y_{H}, c_{0}^{\prime}\left(a_{0}^{e}\left(y_{L}\right)\right)\right) c_{0}^{\prime}\left(a_{0}^{e}\left(y_{L}\right)\right)}{\left(1-q_{H}\right)+q_{H} c_{0}^{\prime}\left(a_{0}^{e}\left(y_{L}\right)\right)} .
$$

Since $b_{0}^{e \prime}\left(Y_{0}, a_{0}^{e}\left(Y_{0}\right)\right) \geq-1, b_{0}^{\prime}\left(a_{0}\right) \geq-1$.

\subsection{Proof of Theorem 2.}

Proof. The proof consists of two steps. First, given the parameters of the securities, we show that the agent's strategy of using all the cash flows to pay the debt replicates the agent's and the investor's continuation payoffs under the corresponding optimal contract $\sigma^{*}$. Then, we show that this strategy is incentive compatible.

Step 1. We assume that the agent adopts the "pay-debt-first" strategy: he uses cash flows generated by the project to pay the credit line balance until it is paid in full, and only after that he starts the consumption. Given this strategy, we will show that, in the credit line settings, for any history of the realizations of cash flows, the payments between the agent and the investor and the termination probabilities are identical to those under the optimal contract $\sigma^{*}$.

To see this, let $a_{t}^{e}\left(Y_{t}\right)=V_{\tau_{t}}\left(Y_{t}\right)$ be the agent's continuation payoffs under the optimal contract, which evolution is determined by the evolution of the earliest default time $\tau_{t}$. 
Consider the following process:

$$
g_{t} \equiv V_{\tau_{t}}\left(y_{H}\right)
$$

One can see that $g_{t}=a_{t}^{e}\left(y_{H}\right)$, and $g_{t}>a_{t}^{e}\left(y_{L}\right)$.

Step 1.1. As an intermediate step, we show by induction that $M_{t}=V_{T-t}\left(y_{H}\right)-g_{t}$. Given $a_{0}^{e}\left(Y_{0}\right)=V_{\tau_{0}}\left(Y_{0}\right)$, the initial draw on the credit line is given by $M_{0}=V_{T}\left(y_{H}\right)-g_{0}$, where $g_{0}=V_{\tau_{0}}\left(y_{H}\right)$. Assume that $M_{t-1}=V_{T-t+1}\left(y_{H}\right)-g_{t-1}=V_{T-t+1}\left(y_{H}\right)-V_{\tau_{t-1}}\left(y_{H}\right)$. The interest rate corresponding to the balance $M_{t-1}$ is given by

$$
r_{t}^{C}=\frac{V_{T-t}\left(y_{H}\right)-V_{\tau_{t-1}-1}\left(y_{H}\right)}{V_{T-t+1}\left(y_{H}\right)-V_{\tau_{t-1}}\left(y_{H}\right)}-1
$$

The agent pays $\left(Y_{t}-y_{L}\right)$ on the credit line in period $t$. The evolution of the balance is given by

$$
M_{t}=\max \left\{0, \min \left\{\left(1+\hat{r}_{t}^{C}\right) M_{t-1}-\left(Y_{t}-y_{L}\right), C_{t}^{L}\right\}\right\} .
$$

Substituting $\hat{r}_{t}^{C}$ and $C_{t}^{L}$ into (94) gives

$$
\begin{aligned}
M_{t} & =\max \left\{0, \min \left\{V_{T-t}\left(y_{H}\right)-V_{\tau_{t-1}-1}\left(y_{H}\right)-\left(Y_{t}-y_{L}\right), V_{T-t}\left(y_{H}\right)-a^{L}\left(y_{H}\right)\right\}\right\} \\
& =\max \left\{0,\left(V_{T-t}\left(y_{H}\right)-\max \left\{V_{\tau_{t-1}-1}\left(y_{H}\right)+\left(Y_{t}-y_{L}\right), a^{L}\left(y_{H}\right)\right)\right\}\right\} \\
& =V_{T-t}\left(y_{H}\right)-\min \left\{V_{T-t}\left(y_{H}\right), \max \left\{V_{\tau_{t-1}-1}\left(y_{H}\right)+\left(Y_{t}-y_{L}\right), a^{L}\left(y_{H}\right)\right\}\right\} .
\end{aligned}
$$

According to (34), $a_{t}^{e}\left(Y_{t}\right)=\min \left\{V_{T-t}\left(Y_{t}\right), \max \left\{V_{\tau_{t-1}-1}\left(Y_{t}\right)+\left(Y_{t}-y_{L}\right), a^{L}\left(Y_{t}\right)\right\}\right\}$, because $a_{t-1}^{e}\left(Y_{t-1}\right)=V_{\tau_{t-1}}\left(Y_{t-1}\right)$. By the definition, if $Y_{t}=y_{H}$, then $g_{t}=a_{t}^{e}\left(y_{H}\right)=$ $\min \left\{V_{T-t}\left(y_{H}\right), \max \left\{V_{\tau_{t-1}-1}\left(y_{H}\right)+y_{H}-y_{L}, V_{1}\left(y_{H}\right)\right\}\right\}$. If $Y_{t}=y_{L}$, then $a_{t}^{e}\left(y_{L}\right)=\min \left\{V_{T-t}\left(y_{L}\right), \max \left\{V_{\tau_{t-1}-1}\left(y_{L}\right), V_{1}\left(y_{L}\right)\right\}\right\}$ and, therefore, $g_{t}=\min \left\{V_{T-t}\left(y_{H}\right), \max \left\{V_{\tau_{t-1}-1}\left(y_{H}\right), V_{1}\left(y_{H}\right)\right\}\right\}$. Thus, $g_{t}=\min \left\{V_{T-t}\left(y_{H}\right), \max \left\{V_{\tau_{t-1}-1}\left(y_{H}\right)+\left(Y_{t}-y_{L}\right), V_{1}\left(y_{H}\right)\right\}\right\}$, which implies that

$$
M_{t}=V_{T-t}\left(y_{H}\right)-g_{t}
$$

\section{Step 1.2}

From (95), one can see that, given the agent's strategy, the credit line replicates the optimal mechanism. A zero balance on the credit line corresponds to the continuation payoff of $a_{t}^{e}\left(Y_{t}\right)=\lambda V_{T-t}\left(Y_{t}\right)$. On the other hand, the liquidation under the credit line implementation happens with probability $\max \{0,(1-\tau)\}$ in period $t$ when $g_{t}=V_{\tau}\left(y_{H}\right)$. This implies that the credit line, combined with the agent's strategy, leads to the same outcome in terms of payments between the agent and the investor and the same termination probabilities as those under the optimal contract.

\section{Step 2.}

To finish the proof we show that the agent's strategy is incentive compatible. Since the 
agent is not allowed to borrow from the credit line for his own consumption, the only possible deviation for him is to use a fraction of the earning for consumption before the credit line is paid off. This deviation is possible only when $Y_{t}=y_{H}$.

Given $Y_{t}=y_{H}$, consider a one-period deviation, i.e. the agent sticks to the "pay-debtfirst" strategy in the subsequent periods. Then, $g_{t}=a_{t}^{e}\left(y_{H}\right)$, and, hence, $M_{t}=a_{t}^{F B}\left(y_{H}\right)-$ $a_{t}^{e}\left(y_{H}\right)$. If $a_{t}^{e}\left(y_{H}\right)>a^{L}\left(y_{H}\right)$, then there is no liquidation. An increase in the credit line balance $M_{t}$ is equivalent to a decrease in the continuation payoff $a_{t}^{e}\left(y_{H}\right)$ by the same amount. Therefore, it is optimal for the agent to pay the credit line first.

Now, consider termination. Let $z_{t}$ be the unmade payment. The agent consumes $z_{t}$, and faces the probability of the termination $p_{t}=\frac{z_{t}}{a^{L}\left(y_{H}\right)}$. With the probability $\left(1-p_{t}\right)$ the unmade payment $z_{t}$ is forgiven. In this case, $M_{t}=C_{t}^{L}=V_{T-t}\left(y_{H}\right)-V_{1}\left(y_{H}\right)$, which implies that agent's continuation payoff is equal to $\lambda V_{1}\left(y_{H}\right)$. The agent's continuation payoff in the middle of period $t$ is, therefore, $\left(1-p_{t}\right) V_{1}\left(y_{H}\right)=V_{1}\left(y_{H}\right)-z_{t}$. One can see that the amount of the unmade payment is subtracted from the continuation payoff of the agent. Thus, the agent does not have any incentive to divert the earning, and the "pay-debt-first" strategy is incentive compatible.

\subsection{Proof of Lemma 4}

One can verify that

$$
E\left[\Delta Y_{t+k} \mid Y_{t}=y\right]=\frac{\Delta}{2}\left(1+(2 y-1)(2 q-1)^{k}\right)
$$

Let $\beta=e^{-r \Delta}, \mu=2 q-1$. Then, using the formula for the sum of a geometric progression yields

$$
E\left[\sum_{k=1}^{\tau / \Delta} e^{-r \Delta k} \Delta Y_{t+k} \mid Y_{t}=y\right] \approx \frac{\Delta}{2}\left(\frac{\beta\left(1-\beta^{\tau / \Delta}\right)}{1-\beta}+(2 y-1) \frac{\beta \mu\left(1-(\beta \mu)^{\tau / \Delta}\right)}{1-\beta \mu}\right)
$$

Taking the limit yields

$$
\begin{aligned}
& \lim _{\Delta \rightarrow 0} \frac{\Delta}{2}\left(\frac{\beta\left(1-\beta^{\tau / \Delta}\right)}{1-\beta}+(2 y-1) \frac{\beta \mu\left(1-(\beta \mu)^{\tau / \Delta}\right)}{1-\beta \mu}\right) \\
= & \lim _{\Delta \rightarrow 0} \frac{e^{-r \Delta}\left(1-e^{-r \tau}\right)}{2\left(1-e^{-r \Delta}\right) / \Delta}+(2 y-1) \lim _{\Delta \rightarrow 0} \frac{e^{-r \Delta}\left(2 e^{-\gamma \Delta}-1\right)\left(1-e^{-r \tau}\left(2 e^{-\gamma \Delta}-1\right)^{\tau / \Delta}\right)}{2\left(1-e^{-r \Delta}\left(2 e^{-\gamma \Delta}-1\right)\right) / \Delta} \\
= & \frac{1-e^{-r \tau}}{2 r}+(2 y-1) \frac{1-e^{-(2 \gamma+r) \tau}}{2(2 \gamma+r)} .
\end{aligned}
$$




\section{References}

[1] Abreu, D., Pearce, D., \& Stacchetti, E. (1990). "Toward a Theory of Discounted Repeated Games with Imperfect Monitoring," Econometrica, 58 (5), pp. 1041-63.

[2] Asquith, P., Beatty, A. and Weber J., (2004) "Performance Pricing in Bank Debt Contracts," Working Paper, MIT and Ohio State University

[3] Battaglini, M. (2004) "Long-Term Contracting with Markovian Consumers," Working Paper, Princeton University

[4] Battaglini, M., Coate, S. (2004) "Pareto Efficient Income Taxation with Stochastic Abilities," Working Paper, Princeton University

[5] Bradley, M., and Roberts, M. (2004) "The Structure and Pricing of Corporate Debt Covenants," Working Paper, Duke University

[6] Bolton, P. and Scharfstein, D. (1990) "A Theory of Predation Based on Agency Problems in Financial Contracting," American Economic Review 80, pp.94-106.

[7] Clementi, G. and Hopenhayn, H. (2002) "A Theory of Financing Contracting and Firm Dynamics," Working Paper, Carnegie Mellon University and University of Rochester

[8] DeMarzo, P. and Fishman, M. (2003) "Optimal Long-Term Financial Contracting with Privately Observed Cash Flows," Working Paper, Northwestern University

[9] DeMarzo, P. and Fishman, M. (2002) "Agency and Optimal Investment Dynamics," Working Paper, Northwestern University

[10] DeMarzo, P. and Sannikov, Y. (2004) "A Continuous-Time Agency Model of Optimal Contracting and Capital Structure," Working Paper, Stanford University

[11] Dichev, I. and Skinner, D. (2004) "Large-sample Evidence on the Debt Covenant Hypothesis," Working Paper, University of Michigan

[12] Diamond, D. W. (1984) "Financial Intermediation and Delegated Monitoring," Review of Economic Studies 51, pp. 394-414.

[13] Doepke, M. \& Townsend, R. M. (2001) "Credit Guarantees, Moral Hazard, and the Optimality of Public Reserves," Unpublished manuscript, UCLA and University of Chicago.

[14] Fernandes, A. and Phelan, C. (2000) "A Recursive Formulation for Repeated Agency with History Dependence," Journal of Economic Theory 91, pp. 223-247 
[15] Green, Edward J. 1987. "Lending and the Smoothing of Uninsurable Income." In Contractual Arrangements for Intertemporal Trade, edited by E. Prescott and N. Wallace, 3-25. Minneapolis: University of Minnesota Press.

[16] Kocherlakota, N. (1996) "Implication of Efficient Risk Sharing without Commitment," Review of Economic Studies 63(4), pp. 595-609.

[17] Manso, G., Strulovici, B. and Tchistyi, A. (2004) "Performance-Sensitive Debt," Working paper, Stanford University

[18] Modigliani, F. and Miller, M. (1958) "The Cost of Capital, Corporation Finance and the Theory of Investment," American Economic Review 48, 267-297

[19] Phelan, C. \& Townsend, R. (1991) "Computing Multiperiod Information-Constrained Optima," Review of Economic Studies, 58 (5), pp. 853-81.

[20] Quadrini V. (2003) "Investment and Liquidation in Renegotiation-Proof Contracts with Moral Hazard," Working Paper, New York University 